\title{
Domino Grignard Addition and Oxidation for the One-Pot Synthesis of C2-Quaternary 2-Hydroxyindoxyls
}

\author{
Tirtha Mandal, Gargi Chakraborti, Subhadip Maiti and Jyotirmayee Dash* \\ School of Chemical Sciences, Indian Association for the Cultivation of Science, Jadavpur, Kolkata \\ 700032, India \\ Correspondence should be addressed to J.D. (ocjd@iacs.res.in)
}

\section{Contents}

1.0 General Information 2

2.0 Synthesis of $N$-protected 3-hydroxy-2-oxindoles 3

3.0 Synthesis of 2-hydroxyindoxyls $\quad 4$

4.0 Gram scale experiment 16

$\begin{array}{lll}5.0 & \text { Deuterium labelling experiment } & 17\end{array}$

$\begin{array}{ll}6.0 & \text { Radical quenching experiment } \\ \end{array}$

7.0 General procedure for the synthesis of C2-allyl 2,2-disubstituted oxindoles 18

8.0 Synthesis of 3-allyl or aryl-3-hydroxy oxindoles 19

9.0 General procedure for the synthesis of 3-aryl or allyl-3-methoxy oxindoles 22

10.0 Synthesis of bis-indoxyl spirofuran derivative 24

$\begin{array}{lll}11.0 & \text { X-Ray Crystallography data } & 25\end{array}$

$\begin{array}{lll}12.0 & \text { References } & 28\end{array}$

13.0 NMR spectra of all compounds $\quad 29$ 


\subsection{General Information}

All experiments were carried out under an inert atmosphere of argon in flame-dried flasks. Solvents were dried using standard procedures. All starting materials were obtained from commercial suppliers and used as received. All the reactions were monitored by thin layer chromatography (TLC) analysis on silica gel 60 F254. Products were purified by flash chromatography on silica gel (100-200 mesh, Merck). Unless otherwise stated, yields refer to analytical pure samples. NMR spectra were recorded in $\mathrm{CDCl}_{3}$ unless otherwise stated. ${ }^{\mathbf{1}} \mathbf{H} \mathbf{~ N M R}$ spectra were recorded at $500 \mathrm{MHz}$ using Brüker AVANCE $500 \mathrm{MHz}$ and JEOL $400 \mathrm{MHz}$ instruments at $298 \mathrm{~K}$. Signals are quoted as $\delta$ values in ppm using residual protonated solvent signals as internal standard $\left(\mathrm{CDCl}_{3}: \delta 7.26 \mathrm{ppm}\right)$. Data is reported as follows: chemical shift, integration, multiplicity $(\mathrm{s}=$ singlet, $\mathrm{d}=$ doublet, $\mathrm{t}=$ triplet, $\mathrm{q}=$ quartet, $\mathrm{p}=$ pentet, $\mathrm{br}=$ broad, $\mathrm{m}$ $=$ multiplet), and coupling constants (Hz). ${ }^{13} \mathbf{C}$ NMR spectra were recorded on either a JEOL-400 (100 MHz), or a Brüker AVANCE $500 \mathrm{MHz}(125 \mathrm{MHz})$ with complete proton decoupling. Chemical shifts $(\delta)$ are reported in ppm downfield from tetramethylsilane with the solvent as the internal reference $\left(\mathrm{CDCl}_{3}: \delta 77.16 \mathrm{ppm}\right)$. HRMS analyses were performed with Q-TOF YA263 high resolution (Water Corporation) instruments by + ve mode electrospray ionization. 


\subsection{Synthesis of $N$-Substituted 3-hydroxy-2-oxindoles 1:}

$N$-Substituted 3-hydroxy-2-oxindoles 1 were prepared from $N$-substituted isatins 6 using reported procedure ${ }^{1}$ by chemoselective $\mathrm{NaBH}_{4}$ reduction at $0{ }^{\circ} \mathrm{C}$-rt for $20 \mathrm{~min}$ (Scheme S1). Products obtained after the workup were sufficiently pure and used in the next step directly from the crude.<smiles>[R1]c1ccc2c(c1)C(=O)C(=O)N2[R16]</smiles>

6a; $R^{1}=H, R^{2}=M e$

6b; $R^{1}=H, R^{2}=E t$

6c; $R^{1}=H, R^{2}=$ allyl

6d; $R^{1}=H, R^{2}=B n$

6e; $R^{1}=H, R^{2}=P M B$

6f; $R^{1}=H, R^{2}=P h$

6g; $\mathrm{R}^{1}=\mathrm{H}, \mathrm{R}^{2}=4-\mathrm{CF}_{3} \mathrm{Ph}$

6h; $\mathrm{R}^{1}=\mathrm{H}, \mathrm{R}^{2}=\left(\mathrm{CH}_{2}\right)_{3} \mathrm{Br}$

6i; $R^{1}=F, R^{2}=M e$

6j; $\mathrm{R}^{1}=\mathrm{Cl}, \mathrm{R}^{2}=\mathrm{Bn}$

$\mathbf{6 k} ; \mathrm{R}^{1}=\mathrm{Br}, \mathrm{R}^{2}=\mathrm{Me}$

6I; $\mathrm{R}^{1}=\mathrm{Br}, \mathrm{R}^{2}=$ allyl

$6 \mathrm{~m} ; \mathrm{R}^{1}=\mathrm{Br}, \mathrm{R}^{2}=\mathrm{Bn}$

$6 n ; R^{1}=I, R^{2}=$ allyl

6o; $R^{1}=7-F, R^{2}=$ allyl

$6 p ; R^{1}=7-\mathrm{Cl}, \mathrm{R}^{2}=$ allyl

6q; $\mathrm{R}^{1}=4,7-\mathrm{Cl}, \mathrm{R}^{2}=$ allyl

6r; $R^{1}=\mathrm{OCF}_{3}, \mathrm{R}^{2}=\mathrm{Me}$

6s; $\mathrm{R}^{1}=\mathrm{OCF}_{3}, \mathrm{R}^{2}=\mathrm{Bn}$

6t; $R^{1}=M e, R^{2}=B n$

$6 u ; R^{1}=5,7-M e, R^{2}=$ allyl

6v; $R^{1}=$ OMe, $R^{2}=$ allyl

$6 w ; R^{1}=5-(4-O M e P h), R^{2}=B n$<smiles></smiles>

1a; $R^{1}=H, R^{2}=M e, 96 \%$

1b; $R^{1}=H, R^{2}=E t, 98 \%$

1c; $R^{1}=H, R^{2}=$ allyl, $94 \%$

1d; $R^{1}=H, R^{2}=B n, 90 \%$

1e; $R^{1}=H, R^{2}=P M B, 88 \%$

1f; $R^{1}=H, R^{2}=P h, 95 \%$

1g; $R^{1}=H, R^{2}=4-C_{3} P h, 90 \%$

1h; $\mathrm{R}^{1}=\mathrm{H}, \mathrm{R}^{2}=\left(\mathrm{CH}_{2}\right)_{3} \mathrm{Br}, 92 \%$

$1 \mathrm{i} ; \mathrm{R}^{1}=\mathrm{F}, \mathrm{R}^{2}=\mathrm{Me}, 90 \%$

$1 \mathrm{j} ; \mathrm{R}^{1}=\mathrm{Cl}, \mathrm{R}^{2}=\mathrm{Bn}, 84 \%$

$1 \mathbf{k} ; R^{1}=B r, R^{2}=M e, 84 \%$

1I; $R^{1}=B r, R^{2}=$ allyl, $80 \%$

$1 \mathrm{~m} ; \mathrm{R}^{1}=\mathrm{Br}, \mathrm{R}^{2}=\mathrm{Bn}, 88 \%$

1n; $R^{1}=$ I, $R^{2}=$ allyl, $78 \%$

1o; $R^{1}=7-F, R^{2}=$ allyl, $92 \%$

1p; $\mathrm{R}^{1}=7-\mathrm{Cl}, \mathrm{R}^{2}=$ allyl, $80 \%$

1q; $\mathrm{R}^{1}=4,7-\mathrm{Cl}, \mathrm{R}^{2}=$ allyl, $80 \%$

$1 \mathbf{r} ; \mathrm{R}^{1}=\mathrm{OCF}_{3}, \mathrm{R}^{2}=\mathrm{Me}, 94 \%$

1s; $\mathrm{R}^{1}=\mathrm{OCF}_{3}, \mathrm{R}^{2}=\mathrm{Bn}, 96 \%$

1t; $R^{1}=M e, R^{2}=B n, 90 \%$

$1 \mathrm{u} ; \mathrm{R}^{1}=5,7-\mathrm{Me}, \mathrm{R}^{2}=$ allyl, $95 \%$

1v; $R^{1}=$ OMe, $R^{2}=$ allyl, $85 \%$

$1 w ; R^{1}=5-(4-O M e P h), R^{2}=B n, 94 \%$

Scheme S1. Synthesis of 3-hydroxy-2-oxindoles 1 from isatins 6. 


\subsection{Synthesis of 2-hydroxyindoxyls 3:}

\subsubsection{General procedure for the synthesis of 2-hydroxyindoxyls 3a-3b' (GP-1):}

To a stirring solution of $N$-substituted 3-hydroxy-2-oxindoles 1 (1 equiv.) in dry THF, Grignard reagent solution (4 equiv., 1M in THF; for allyl Grignard 3 equiv. was used) was added dropwise at room temperature. The reaction mixture was then stirred at room temperature for 6 h. After completion of reaction as monitored by the TLC, the reaction mixture was quenched with saturated aqueous $\mathrm{NH}_{4} \mathrm{Cl}$ solution and then extracted with EtOAc $(3 \times 20 \mathrm{~mL})$. The combined organic phases were washed with brine, dried over anhydrous $\mathrm{Na}_{2} \mathrm{SO}_{4}$, filtered and concentrated in vacuo. The crude residue was then purified by column chromatography on silica gel with ethylacetate-hexane (10/90 to 20/80) to give compounds $\mathbf{3 a - 3} \mathbf{3} \mathbf{b}^{\prime}$.

2-Hydroxy-1-methyl-2-phenylindolin-3-one (3a): Using the general procedure GP-1, compound 1a (200 mg, $1.23 \mathrm{mmol})$ and phenylmagnesium bromide $2 \mathrm{a}(5.0 \mathrm{~mL}, 4.92 \mathrm{mmol})$ provided

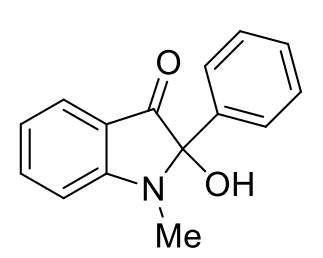
compound 3a (218 mg, 74\%) as a reddish brown solid; ${ }^{1}$ H-NMR (400 $\left.\mathrm{MHz}, \mathrm{CDCl}_{3}\right):$ 7.55-7.51 (2H, m), 7.42-7.39 (2H, m), 7.36-7.33 (3H, m), 6.77-6.74 (2H, m), $3.46(1 \mathrm{H}, \mathrm{sbr}), 2.84(3 \mathrm{H}, \mathrm{s}) ;{ }^{13} \mathrm{C}-\mathrm{NMR}(100 \mathrm{MHz}$, $\left.\mathrm{CDCl}_{3}\right)$ : 200.0, 161.3, 138.9, 136.0, 129.0, 128.9, 126.2, 126.1, 118.2, 117.4, 108.0, 91.0, 27.6; HRMS (ESI) calcd for $\mathrm{C}_{15} \mathrm{H}_{13} \mathrm{NNaO}_{2}[\mathrm{M}+\mathrm{Na}]^{+}$: 262.0844; Found: 262.0844 .

2-Hydroxy-1-allyl-2-phenylindolin-3-one (3b): Using the general procedure GP-1, compound 1c $(200 \mathrm{mg}, 1.06 \mathrm{mmol})$ and phenylmagnesium bromide $2 \mathrm{a}(4.2 \mathrm{~mL}, 4.24 \mathrm{mmol})$ provided

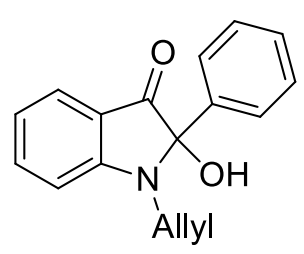
compound $\mathbf{3 b}(220 \mathrm{mg}, 78 \%)$ as a brown gummy liquid; ${ }^{1} \mathbf{H}-\mathbf{N M R}(500$ $\left.\mathrm{MHz}, \mathrm{CDCl}_{3}\right): 7.54(1 \mathrm{H}, \mathrm{d}, J=7.6 \mathrm{~Hz}), 7.50(1 \mathrm{H}, \mathrm{t}, J=8.4 \mathrm{~Hz}), 7.43-7.41$ $(2 \mathrm{H}, \mathrm{m}), 7.35-7.33(3 \mathrm{H}, \mathrm{m}), 6.78-6.76(2 \mathrm{H}, \mathrm{m}), 5.84-5.76(1 \mathrm{H}, \mathrm{m}), 5.22(1 \mathrm{H}$, dd, $J=15.8,1.9 \mathrm{~Hz}), 5.14(1 \mathrm{H}, \mathrm{d}, J=10.7 \mathrm{~Hz}), 3.90(1 \mathrm{H}, \mathrm{dd}, J=10.7,6.3$ Hz), 3.82-3.78 (1H, m), $3.44(1 \mathrm{H}, \mathrm{d}, J=10.7 \mathrm{~Hz}) ;{ }^{13} \mathbf{C}-\mathbf{N M R}\left(100 \mathrm{MHz}, \mathrm{CDCl}_{3}\right)$ : 200.0, 160.7, 138.7, 136.6, 134.4, 129.0, 128.9, 126.3, 126.1, 118.4, 117.5, 117.1, 109.0, 91.3, 45.5; HRMS (ESI) calcd for $\mathrm{C}_{17} \mathrm{H}_{15} \mathrm{NNaO}_{2}[\mathrm{M}+\mathrm{Na}]^{+}: 288.1000$; Found: 288.1003 . 
2-Hydroxy-1-(4-methoxybenzyl)-2-phenylindolin-3-one (3c): Using the general procedure GP1, compound 1e (200 mg, $0.74 \mathrm{mmol})$ and phenylmagnesium bromide 2a $(3.0 \mathrm{~mL}, 2.96 \mathrm{mmol})$

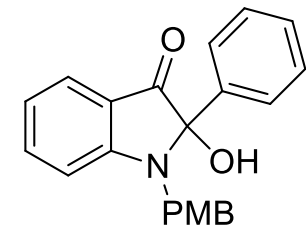
provided compound 3c (195 mg, 76\%) as a yellow solid; ${ }^{1} \mathbf{H}-\mathbf{N M R}$ (400 $\left.\mathrm{MHz}, \mathrm{CDCl}_{3}\right): 7.57(1 \mathrm{H}, \mathrm{d}, J=7.5 \mathrm{~Hz}), 7.45-7.42(3 \mathrm{H}, \mathrm{m}), 7.40-7.35(3 \mathrm{H}$, m), $7.22(2 \mathrm{H}, \mathrm{d}, J=8.6 \mathrm{~Hz}), 6.82(2 \mathrm{H}, \mathrm{d}, J=8.6 \mathrm{~Hz}), 6.77(1 \mathrm{H}, \mathrm{t}, J=7.5$ Hz), $6.60(1 \mathrm{H}, \mathrm{d}, J=8.6 \mathrm{~Hz}), 4.34(2 \mathrm{H}, \mathrm{q}, J=4.3 \mathrm{~Hz}), 3.77(3 \mathrm{H}, \mathrm{s}), 3.25$ $(1 \mathrm{H}, \mathrm{Sbr}) ;{ }^{13} \mathrm{C}-\mathbf{N M R}\left(100 \mathrm{MHz}, \mathrm{CDCl}_{3}\right):$ 199.8, 160.8, 159.0, 138.7, 136.7, 129.8, 129.1, 129.0, 128.5, 126.2, 126.1, 118.7, 117.8, 114.2, 109.1, 91.7, 55.4, 46.8; HRMS (ESI) calcd for $\mathrm{C}_{22} \mathrm{H}_{20} \mathrm{NO}_{3}[\mathrm{M}+\mathrm{H}]^{+}:$346.1443; Found: 346.1439.

2-Hydroxy-1-phenyl-2-phenylindolin-3-one (3d): Using the general procedure GP-1, compound 1f $(200 \mathrm{mg}, 0.89 \mathrm{mmol})$ and phenylmagnesium bromide $\mathbf{2 a}(3.5 \mathrm{~mL}, 3.56 \mathrm{mmol})$ provided

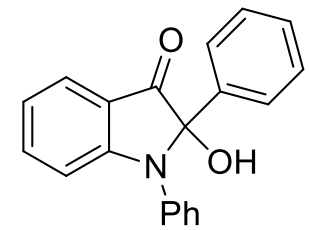
compound 3d (194 mg, 72\%) as a deep brown liquid; ${ }^{\mathbf{H}} \mathbf{H}-\mathbf{N M R}(500 \mathrm{MHz}$, $\left.\mathrm{CDCl}_{3}\right): 7.65(1 \mathrm{H}, \mathrm{d}, J=7.5 \mathrm{~Hz}), 7.58-7.49(2 \mathrm{H}, \mathrm{m}), 7.47-7.40(3 \mathrm{H}, \mathrm{m})$, 7.31-7.25 (5H, m), $7.13(1 \mathrm{H}, \mathrm{t}, J=7.6 \mathrm{~Hz}), 7.08(1 \mathrm{H}, \mathrm{d}, J=8.4 \mathrm{~Hz}), 6.89$ $(1 \mathrm{H}, \mathrm{t}, J=7.6 \mathrm{~Hz}), 3.74(1 \mathrm{H}, \mathrm{sbr}) ;{ }^{13} \mathbf{C}-\mathbf{N M R}\left(125 \mathrm{MHz}, \mathrm{CDCl}_{3}\right): 199.7$, $159.4,138.5,136.9,130.1,129.5,128.9,128.7,126.3,125.0,119.9,118.3$, 111.0, 91.7; HRMS (ESI) calcd for $\mathrm{C}_{20} \mathrm{H}_{16} \mathrm{NO}_{2}[\mathrm{M}+\mathrm{H}]^{+}: 302.1181$; Found: 302.1180 .

2-Hydroxy-2-phenyl-1-(4-(trifluoromethyl)phenyl)indolin-3-one (3e): Using the general procedure GP-1, compound $\mathbf{1 g}(200 \mathrm{mg}, 0.68 \mathrm{mmol})$ and phenylmagnesium bromide $\mathbf{2 a}(2.7$<smiles>O=C1c2ccccc2N(c2ccc(C(F)(F)F)cc2)C1(O)c1ccccc1</smiles>
$\mathrm{mL}, 2.72 \mathrm{mmol})$ provided compound $\mathbf{3 e}(186 \mathrm{mg}, 74 \%)$ as a greenish liquid; ${ }^{1} \mathrm{H}-\mathrm{NMR}\left(500 \mathrm{MHz}, \mathrm{CDCl}_{3}\right): 7.74(1 \mathrm{H}, \mathrm{d}, J=8.2 \mathrm{~Hz}), 7.66(1 \mathrm{H}, \mathrm{s}), 7.62-$ $7.54(2 \mathrm{H}, \mathrm{m}), 7.52-7.50(1 \mathrm{H}, \mathrm{m}), 7.45-7.43$ (2H, m), 7.40-7.38 (2H, m), 7.33$7.29(2 \mathrm{H}, \mathrm{m}), 7.16(1 \mathrm{H}, \mathrm{d}, J=8.2 \mathrm{~Hz}), 7.01(1 \mathrm{H}, \mathrm{t}, J=7.6 \mathrm{~Hz}), 3.74(1 \mathrm{H}$, Sbr); ${ }^{13}$ C-NMR (100 MHz, $\left.\mathrm{CDCl}_{3}\right): 199.2,158.3,139.3,138.7,136.4,129.9$, $129.2,128.9,127.5,126.5,126.1,122.3,121.1,121.0,120.9,118.7,110.9$, 91.8; HRMS (ESI) calcd for $\mathrm{C}_{21} \mathrm{H}_{14} \mathrm{~F}_{3} \mathrm{NNaO}_{2}$ [M+Na] $]^{+}$: 392.0874; Found: 392.0875. 
1-(3-Bromopropyl)-2-hydroxy-2-phenylindolin-3-one (3f): Using the general procedure GP-1, compound $\mathbf{1 h}(200 \mathrm{mg}, 0.74 \mathrm{mmol})$ and phenylmagnesium bromide $\mathbf{2 a}(3.0 \mathrm{~mL}, 2.96 \mathrm{mmol})$

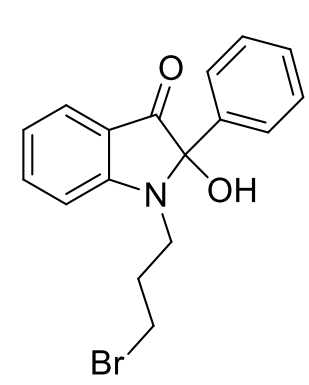
provided compound $\mathbf{3 f}(180 \mathrm{mg}, 70 \%)$ as a brown liquid; ${ }^{1} \mathbf{H}-\mathbf{N M R}$ (500 $\left.\mathrm{MHz}_{\mathrm{CDCl}}\right): 7.56-7.50(2 \mathrm{H}, \mathrm{m}), 7.39-7.32(5 \mathrm{H}, \mathrm{m}), 6.88(1 \mathrm{H}, \mathrm{d}, J=8.3$ $\mathrm{Hz}), 6.76(1 \mathrm{H}, \mathrm{t}, J=7.8 \mathrm{~Hz}), 3.91(1 \mathrm{H}, \mathrm{sbr}), 3.62-3.54(1 \mathrm{H}, \mathrm{m}), 3.45-3.36$ $(2 \mathrm{H}, \mathrm{m}), 3.27-3.20(1 \mathrm{H}, \mathrm{m}), 2.13-2.06(2 \mathrm{H}, \mathrm{m}) ;{ }^{13} \mathrm{C}-\mathrm{NMR}(100 \mathrm{MHz}$, $\left.\mathrm{CDCl}_{3}\right): 200.3,160.9,139.0,136.5,129.0,128.9,126.3,126.1,118.4,117.3$, 108.3, 91.4, 41.2, 31.8, 31.4; HRMS (ESI) calcd for $\mathrm{C}_{17} \mathrm{H}_{17} \mathrm{BrNO}_{2}[\mathrm{M}+\mathrm{H}]^{+}$: 346.0443; Found: 346.0449.

5-Fluoro-2-hydroxy-1-methyl-2-phenylindolin-3-one (3g): Using the general procedure GP-1, compound 1i (200 mg, $1.10 \mathrm{mmol})$ and phenylmagnesium bromide 2a (4.4 mL, $4.40 \mathrm{mmol})$<smiles>CN1c2ccc(F)cc2C(=O)C1(O)c1ccccc1</smiles>
provided compound $\mathbf{3 g}(216 \mathrm{mg}, 76 \%)$ as a red solid; ${ }^{\mathbf{1}} \mathbf{H}-\mathbf{N M R}$ (500 $\left.\mathrm{MHz}_{\mathrm{CDCl}}\right)$ : 7.40-7.35 (5H, m), 7.31-7.25 (1H, m), 7.20-7.18 (1H, m), 6.72-6.69 (1H, m), $3.49(1 \mathrm{H}, \mathrm{sbr}), 2.82(3 \mathrm{H}, \mathrm{s}) ;{ }^{13} \mathbf{C}-\mathbf{N M R}(125 \mathrm{MHz}$, $\left.\mathrm{CDCl}_{3}\right): 199.7,158.1,157.0,155.1,135.7,129.1,129.0,126.6,126.4$, 126.1, 117.6, 117.5, 111.3, 111.1, 107.0, 108.9, 91.6, 27.8; HRMS (ESI) calcd for $\mathrm{C}_{15} \mathrm{H}_{12} \mathrm{FNNaO}_{2}[\mathrm{M}+\mathrm{Na}]^{+}: 280.0750$; Found: 280.0752 .

7-Fluoro-2-hydroxy-1-allyl-2-phenylindolin-3-one (3h): Using the general procedure GP-1, compound 10 (200 mg, $0.97 \mathrm{mmol})$ and phenylmagnesium bromide $\mathbf{2 a}(3.9 \mathrm{~mL}, 3.88 \mathrm{mmol})$<smiles>O=C1c2cccc(F)c2N([AlH2])C1(O)c1ccccc1</smiles>
provided compound $\mathbf{3 h}$ (195 mg, 71\%) as a brown gummy liquid; ${ }^{\mathbf{1}} \mathbf{H}$-NMR $\left(400 \mathrm{MHz}, \mathrm{CDCl}_{3}\right): 7.48-7.46(2 \mathrm{H}, \mathrm{m}), 7.36-7.32(4 \mathrm{H}, \mathrm{m}), 7.23(1 \mathrm{H}, \mathrm{t}, J=$ $4.4 \mathrm{~Hz}), 6.70-6.65(1 \mathrm{H}, \mathrm{m}), 5.94-5.84(1 \mathrm{H}, \mathrm{m}), 5.16(1 \mathrm{H}, \mathrm{d}, J=17.6 \mathrm{~Hz})$, $5.05(1 \mathrm{H}, \mathrm{d}, J=10.3 \mathrm{~Hz}), 4.22(1 \mathrm{H}, \mathrm{dd}, J=10.8,5.8 \mathrm{~Hz}), 3.86(1 \mathrm{H}, \mathrm{dd}, J=$ 11.2, 4.9 Hz), OH could not be detected; ${ }^{13} \mathbf{C}-\mathbf{N M R}\left(100 \mathrm{MHz}, \mathrm{CDCl}_{3}\right): 199.4,149.7,148.1$, 148.0, 147.3, 136.3, 135.6, 129.3, 128.9, 126.3, 125.1, 124.9, 121.9, 121.8, 121.1, 121.0, 118.5, 118.4, 116.8, 91.5, 46.6, 46.5; HRMS (ESI) calcd for $\mathrm{C}_{17} \mathrm{H}_{15} \mathrm{FNO}_{2}[\mathrm{M}+\mathrm{H}]^{+}$: 284.1087; Found: 284.1092. 
1-Allyl-4,7-dichloro-2-hydroxy-2-phenylindolin-3-one (3i): Using the general procedure GP-1, compound 1q (200 mg, $0.77 \mathrm{mmol})$ and phenylmagnesium bromide $\mathbf{2 a}(3.1 \mathrm{~mL}, 3.08 \mathrm{mmol})$<smiles>O=C1c2c(Cl)ccc(Cl)c2N([AlH2])C1(O)c1ccccc1</smiles>
provided compound $\mathbf{3 i}$ (65 mg, 64\%) as a brown liquid; ${ }^{1} \mathbf{H}-\mathbf{N M R}(400 \mathrm{MHz}$, $\left.\mathrm{CDCl}_{3}\right): 7.49-7.46(1 \mathrm{H}, \mathrm{m}), 7.42(1 \mathrm{H}, \mathrm{d}, J=8.8 \mathrm{~Hz}), 7.39-7.26(4 \mathrm{H}, \mathrm{m}), 7.02$ $(1 \mathrm{H}, \mathrm{d}, J=8.8 \mathrm{~Hz}), 6.00-5.91(1 \mathrm{H}, \mathrm{m}), 5.26(1 \mathrm{H}, \mathrm{s}), 5.23(1 \mathrm{H}, \mathrm{d}, J=5.4 \mathrm{~Hz})$, 4.78-4.76 (2H, m); ${ }^{13} \mathbf{C}-\mathbf{N M R}\left(100 \mathrm{MHz}, \mathrm{CDCl}_{3}\right): 196.5,157.7,140.7$, 140.3, 136.3, 131.7, 129.5, 129.0, 126.7, 126.5, 126.4, 120.5, 118.0, 91.8, 43.9; HRMS (ESI) calcd for $\mathrm{C}_{17} \mathrm{H}_{14} \mathrm{Cl}_{2} \mathrm{NO}_{2}[\mathrm{M}+\mathrm{H}]^{+}$: 334.0402; Found: 334.0399 .

1-Benzyl-5-bromo-2-hydroxy-2-phenylindolin-3-one (3j): Using the general procedure GP-1, compound $1 \mathbf{m}$ (200 mg, $0.63 \mathrm{mmol})$ and phenylmagnesium bromide $\mathbf{2 a}(3.1 \mathrm{~mL}, 2.52 \mathrm{mmol})$<smiles>O=C1c2cc(Br)ccc2N(Cc2ccccc2)C1(O)c1ccccc1</smiles>
provided compound $\mathbf{3 j}$ (172 $\mathrm{mg}, 68 \%$ ) as a brown liquid; ${ }^{1} \mathbf{H}-\mathbf{N M R}(400$ $\left.\mathrm{MHz}, \mathrm{CDCl}_{3}\right)$ : $7.54(1 \mathrm{H}, \mathrm{s}), 7.45-7.42(3 \mathrm{H}, \mathrm{m}), 7.31-7.35(3 \mathrm{H}, \mathrm{m}), 7.32-$ $7.23(5 \mathrm{H}, \mathrm{m}), 6.44(1 \mathrm{H}, \mathrm{d}, J=8.8 \mathrm{~Hz}), 4.38(2 \mathrm{H}, \mathrm{dd}, J=16.6,8.3 \mathrm{~Hz})$, $4.16\left(1 \mathrm{H}, \mathrm{sbr}_{\mathrm{r}}\right) ;{ }^{13} \mathrm{C}-\mathbf{N M R}\left(100 \mathrm{MHz}, \mathrm{CDCl}_{3}\right): 199.0,159.3,141.1,137.3$, 136.1, 129.3, 129.1, 128.9, 128.3, 127.5, 127.2, 126.2, 119.3, 110.8, 110.6, 92.1, 47.2; HRMS (ESI) calcd for $\mathrm{C}_{21} \mathrm{H}_{16} \mathrm{BrNNaO}_{2}[\mathrm{M}+\mathrm{Na}]^{+}$: 416.0262; Found: 416.0264 .

1-Allyl-2-hydroxy-5-iodo-2-phenylindolin-3-one (3k): Using the general procedure GP-1, compound 1n (200 mg, $0.63 \mathrm{mmol})$ and phenylmagnesium bromide $2 \mathrm{a}(2.5 \mathrm{~mL}, 2.52 \mathrm{mmol})$<smiles>O=C1c2cc(I)ccc2N([Al])C1(O)c1ccccc1</smiles>
provided compound 3k (174 mg, 70\%) as a deep green liquid; ${ }^{\mathbf{1}} \mathbf{H}-\mathbf{N M R}$ $\left(500 \mathrm{MHz}, \mathrm{CDCl}_{3}\right): 7.73(1 \mathrm{H}, \mathrm{d}, J=1.9 \mathrm{~Hz}), 7.70(1 \mathrm{H}, \mathrm{dd}, J=6.9,1.9$ $\mathrm{Hz}), 7.40-7.32(5 \mathrm{H}, \mathrm{m}), 6.58(1 \mathrm{H}, \mathrm{d}, J=8.8 \mathrm{~Hz}), 5.79-5.71(1 \mathrm{H}, \mathrm{m}), 5.22$ $(1 \mathrm{H}, \mathrm{dd}, J=15.8,1.3 \mathrm{~Hz}), 5.14(1 \mathrm{H}, \mathrm{dd}, J=8.8,1.3 \mathrm{~Hz}), 3.95(1 \mathrm{H}, \mathrm{sbr})$, $3.88(1 \mathrm{H}, \mathrm{dd}, J=16.4,6.4 \mathrm{~Hz}), 3.81-3.76(1 \mathrm{H}, \mathrm{m}) ;{ }^{13} \mathbf{C}-\mathbf{N M R}\left(100 \mathrm{MHz}, \mathrm{CDCl}_{3}\right): 198.3,159.7$, 146.6, 136.0, 134.4, 133.9, 129.2, 128.9, 126.2, 119.8, 117.5, 111.3, 91.4, 78.9, 45.4; HRMS (ESI) calcd for $\mathrm{C}_{17} \mathrm{H}_{14} \mathrm{INNaO}_{2}[\mathrm{M}+\mathrm{Na}]^{+}$: 413.9967; Found: 413.9966. 
2-Hydroxy-1-methyl-2-phenyl-5-(trifluoromethoxy)indolin-3-one (31): Using the general procedure GP-1, compound 1r (200 $\mathrm{mg}, 0.81 \mathrm{mmol})$ and phenylmagnesium bromide $\mathbf{2 a}(3.2 \mathrm{~mL}$,<smiles>CN1c2ccc(OC(F)(F)F)cc2C(=O)C1(O)c1ccccc1</smiles>

$3.24 \mathrm{mmol}$ ) provided compound $\mathbf{3 l}$ (178 $\mathrm{mg}, 68 \%)$ as a brown gummy solid; ${ }^{1} \mathrm{H}-\mathrm{NMR}\left(400 \mathrm{MHz}, \mathrm{CDCl}_{3}\right):$ 7.41-7.36 (7H, m), $6.75(1 \mathrm{H}, \mathrm{d}, J$ $=9.3 \mathrm{~Hz}), 3.33(1 \mathrm{H}, \mathrm{sbr}), 2.87(3 \mathrm{H}, \mathrm{s}) ;{ }^{13} \mathbf{C}-\mathbf{N M R}\left(100 \mathrm{MHz}, \mathrm{CDCl}_{3}\right)$ : 199.1, 159.6, 141.1, 135.4, 132.4, 129.3, 129.1, 126.1, 118.7, 117.6, 108.8, 91.5, 27.7; HRMS (ESI) calcd for $\mathrm{C}_{16} \mathrm{H}_{13} \mathrm{~F}_{3} \mathrm{NO}_{3}[\mathrm{M}+\mathrm{H}]^{+}$: 324.0848; Found: 324.0855.

1-Benzyl-2-hydroxy-5-methyl-2-phenylindolin-3-one (3m): Using the general procedure GP-1, compound 1t (200 mg, $0.79 \mathrm{mmol})$ and phenylmagnesium bromide $2 \mathbf{a}(3.2 \mathrm{~mL}, 3.16 \mathrm{mmol})$<smiles>Cc1ccc2c(c1)C(=O)C(O)(c1ccccc1)N2Cc1ccccc1</smiles>
provided compound $\mathbf{3 m}$ (193 mg, 74\%) as a brown gummy solid; ${ }^{1} \mathbf{H}-$ NMR $\left(400 \mathrm{MHz}, \mathrm{CDCl}_{3}\right): 7.46-7.43(2 \mathrm{H}, \mathrm{m}), 7.38-7.34(5 \mathrm{H}, \mathrm{m}), 7.31-$ $7.29(3 \mathrm{H}, \mathrm{m}), 7.27-7.22(2 \mathrm{H}, \mathrm{m}), 6.48(1 \mathrm{H}, \mathrm{d}, J=8.5 \mathrm{~Hz}), 4.37(2 \mathrm{H}, \mathrm{s})$, $3.64\left(1 \mathrm{H}, \mathrm{sbr}_{\mathrm{r}}\right), 2.22(3 \mathrm{H}, \mathrm{s}) ;{ }^{13} \mathrm{C}-\mathrm{NMR}\left(125 \mathrm{MHz}, \mathrm{CDCl}_{3}\right): 200.1,159.3$, 139.9, 138.2, 136.9, 128.9, 128.7, 128.1, 127.3, 126.3, 125.6, 117.9, 109.0, 92.1, 47.4, 20.4; HRMS (ESI) calcd for $\mathrm{C}_{22} \mathrm{H}_{19} \mathrm{NNaO}_{2}[\mathrm{M}+\mathrm{Na}]^{+}$: 352.1313; Found: 352.1314 .

1-Allyl-2-hydroxy-5,7-dimethyl-2-phenylindolin-3-one (3n): Using the general procedure GP-1, compound $1 \mathbf{u}(200 \mathrm{mg}, 0.92 \mathrm{mmol})$ and phenylmagnesium bromide $\mathbf{2 a}(3.7 \mathrm{~mL}, 3.68 \mathrm{mmol})$

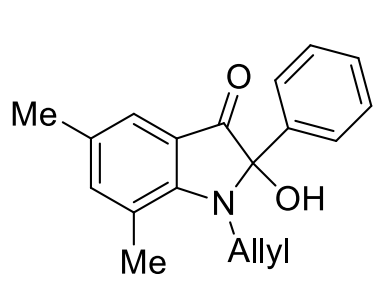
provided compound $3 \mathbf{n}$ (210 $\mathrm{mg}, 76 \%)$ as a greenish yellow liquid; ${ }^{1} \mathbf{H}-$ NMR (500 MHz, $\left.\mathrm{CDCl}_{3}\right): 7.50-7.46(2 \mathrm{H}, \mathrm{m}), 7.35-7.30(3 \mathrm{H}, \mathrm{m}), 7.21$ $(1 \mathrm{H}, \mathrm{s}), 7.11(1 \mathrm{H}, \mathrm{s}), 5.98-5.91(1 \mathrm{H}, \mathrm{m}), 5.18-5.13(1 \mathrm{H}, \mathrm{m}), 5.10-5.07$ $(1 \mathrm{H}, \mathrm{m}), 4.34-4.28(1 \mathrm{H}, \mathrm{m}), 3.90-3.85(1 \mathrm{H}, \mathrm{m}), 3.32(1 \mathrm{H}, \mathrm{sbr}), 2.48(3 \mathrm{H}$, s), $2.22(3 \mathrm{H}, \mathrm{s}) ;{ }^{13} \mathrm{C}-\mathrm{NMR}\left(100 \mathrm{MHz}, \mathrm{CDCl}_{3}\right)$ : 200.5, 157.9, 143.3, 137.8, 137.4, 128.9, 128.8, 126.4 123.4, 120.0, 118.6, 115.7, 91.7, 46.1, 20.2, 19.4; HRMS (ESI) calcd for $\mathrm{C}_{19} \mathrm{H}_{19} \mathrm{NNaO}_{2}$ $[\mathrm{M}+\mathrm{Na}]^{+}: 316.1313$; Found: 316.1314 . 
1-Benzyl-2-hydroxy-5-(4-methoxyphenyl)-2-phenylindolin-3-one (3o): Using the general procedure GP-1, compound $\mathbf{1}$ w (200 $\mathrm{mg}, 0.58 \mathrm{mmol}$ ) and allylmagnesium bromide $2 \mathbf{c}$ ( $2.3 \mathrm{~mL}$,

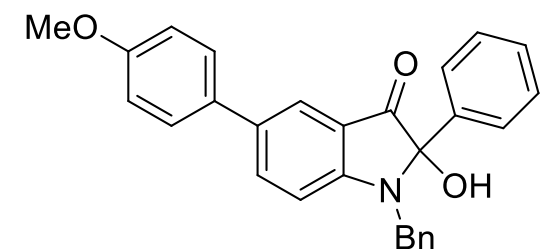
$2.32 \mathrm{mmol})$ provided compound $30(160 \mathrm{mg}, 66 \%)$ as a greenish yellow liquid; ${ }^{1} \mathrm{H}-\mathrm{NMR}\left(500 \mathrm{MHz}, \mathrm{CDCl}_{3}\right): 7.75$ $(1 \mathrm{H}, \mathrm{d}, J=1.9 \mathrm{~Hz}), 7.62(1 \mathrm{H}, \mathrm{dd}, J=6.9,1.9 \mathrm{~Hz}), 7.48(2 \mathrm{H}$, dd, $J=5.7,2.5 \mathrm{~Hz}), 7.40-7.37(5 \mathrm{H}, \mathrm{m}), 7.35-7.31(5 \mathrm{H}, \mathrm{m})$, $6.92(2 \mathrm{H}, \mathrm{d}, J=8.8 \mathrm{~Hz}), 6.62(1 \mathrm{H}, \mathrm{d}, J=8.2 \mathrm{~Hz}), 4.43(2 \mathrm{H}, \mathrm{s}), 3.83(3 \mathrm{H}, \mathrm{s}), 3.54(1 \mathrm{H}, \mathrm{sbr}) ;{ }^{13} \mathrm{C}-$ NMR (100 MHz, $\left.\mathrm{CDCl}_{3}\right):$ 200.0, 159.8, 159.1, 137.9, 137.6, 136.6, 132.6, 131.9, 129.2, 129.1, $128.8,127.5,127.4,127.3,126.3,123.4,118.3,114.5,109.5,92.2,55.5,47.4$; HRMS (ESI) calcd for $\mathrm{C}_{28} \mathrm{H}_{23} \mathrm{NNaO}_{3}[\mathrm{M}+\mathrm{Na}]^{+}:$:44.1576; Found: 444.1578.

1,2-Diallyl-2-hydroxy-5-methoxyindolin-3-one (3p): Using the general procedure GP-1, compound 1v (200 mg, $0.91 \mathrm{mmol})$ and allylmagnesium bromide $2 \mathbf{c}(2.7 \mathrm{~mL}, 2.73 \mathrm{mmol})$<smiles>C=CCC1(O)C(=O)c2cc(OC)ccc2N1[Mg]</smiles>
provided compound 3p (164 mg, 69\%) as a greenish yellow liquid; ${ }^{1} \mathbf{H}$ NMR $\left(400 \mathrm{MHz}, \mathrm{CDCl}_{3}\right): 7.10(1 \mathrm{H}, \mathrm{dd}, J=6.4,2.4 \mathrm{~Hz}), 6.96(1 \mathrm{H}, \mathrm{d}$, $J=2.4 \mathrm{~Hz}), 6.64(1 \mathrm{H}, \mathrm{d}, J=8.8 \mathrm{~Hz}), 5.95-5.86(1 \mathrm{H}, \mathrm{m}), 5.62-5.52$ $(1 \mathrm{H}, \mathrm{m}), 5.32-5.19(2 \mathrm{H}, \mathrm{m}), 5.13-5.01(2 \mathrm{H}, \mathrm{m}), 4.05-3.90(2 \mathrm{H}, \mathrm{m})$, $3.73(3 \mathrm{H}, \mathrm{s}), 3.18(1 \mathrm{H}, \mathrm{sbr}), 2.67-2.59(2 \mathrm{H}, \mathrm{m}) ;{ }^{13} \mathrm{C}-\mathrm{NMR}\left(125 \mathrm{MHz}, \mathrm{CDCl}_{3}\right): 200.1,156.5$, 152.6, 134.9, 130.6, 128.6, 120.1, 117.9, 116.9, 110.6, 105.9, 90.1, 56.0, 44.7, 40.0; HRMS (ESI) calcd for $\mathrm{C}_{15} \mathrm{H}_{18} \mathrm{NO}_{3}[\mathrm{M}+\mathrm{H}]^{+}:$260.1287; Found: 260.1289 .

2-Allyl-1-ethyl-2-hydroxyindolin-3-one (3q): Using the general procedure GP-1, compound 1b (200 $\mathrm{mg}, 1.13 \mathrm{mmol})$ and allylmagnesium bromide $2 \mathrm{c}(3.4 \mathrm{~mL}, 3.39 \mathrm{mmol})$ provided compound<smiles>C=CCC1(O)C(=O)c2ccccc2N1CC</smiles>

3q (184 mg, 75\%) as a yellow liquid; ${ }^{1} \mathbf{H}-\mathbf{N M R}\left(400 \mathrm{MHz}, \mathrm{CDCl}_{3}\right): 7.51$ $(1 \mathrm{H}, \mathrm{d}, J=7.8 \mathrm{~Hz}), 7.45(1 \mathrm{H}, \mathrm{t}, J=8.3 \mathrm{~Hz}), 6.70-6.67(2 \mathrm{H}, \mathrm{m}), 5.57-5.49$ $(1 \mathrm{H}, \mathrm{m}), 5.11(1 \mathrm{H}, \mathrm{d}, J=17.1 \mathrm{~Hz}), 5.02(1 \mathrm{H}, \mathrm{d}, J=10.2 \mathrm{~Hz}), 3.54-3.38(2 \mathrm{H}$, $\mathrm{m}), 2.71-2.62(2 \mathrm{H}, \mathrm{m}), 1.30(3 \mathrm{H}, \mathrm{t}, J=6.8 \mathrm{~Hz})$; OH could not be detected; ${ }^{13}$ C-NMR (125 MHz, $\left.\mathrm{CDCl}_{3}\right)$ : 201.2, 160.1, 138.7, 130.4, 125.5, 120.1, 117.6, 108.3, 89.5, 39.8, 36.1, 14.6; HRMS (ESI) calcd for $\mathrm{C}_{13} \mathrm{H}_{15} \mathrm{NNaO}_{2}[\mathrm{M}+\mathrm{Na}]^{+}: 240.1001$; Found: 240.1004. 
1,2-Diallyl-2-hydroxyindolin-3-one (3r): Using the general procedure GP-1, compound 1c (200 $\mathrm{mg}, 1.05 \mathrm{mmol})$ and allylmagnesium bromide $2 \mathbf{c}(3.2 \mathrm{~mL}, 3.17 \mathrm{mmol})$ provided compound $\mathbf{3 r}$

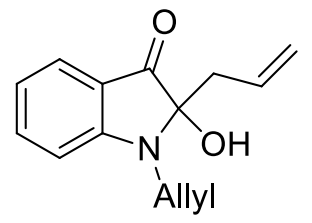

(194 mg, 80\%) as a yellow liquid; ${ }^{1} \mathbf{H}-\mathbf{N M R}\left(500 \mathrm{MHz}, \mathrm{CDCl}_{3}\right): 7.54(1 \mathrm{H}$, d, $J=7.4 \mathrm{~Hz}), 7.46-7.42(1 \mathrm{H}, \mathrm{m}), 6.73(1 \mathrm{H}, \mathrm{t}, J=7.4 \mathrm{~Hz}), 6.68(1 \mathrm{H}, \mathrm{d}, J=$ $7.9 \mathrm{~Hz}), 5.95-5.88(1 \mathrm{H}, \mathrm{m}), 5.64-5.56(1 \mathrm{H}, \mathrm{m}), 5.30(1 \mathrm{H}, \mathrm{dd}, J=15.9,1.2$ $\mathrm{Hz}), 5.22(1 \mathrm{H}, \mathrm{dd}, J=9.2,1.3 \mathrm{~Hz}), 5.14(1 \mathrm{H}, \mathrm{dd}, J=15.9,1.2 \mathrm{~Hz}), 5.05$ $(1 \mathrm{H}, \mathrm{d}, J=10.9 \mathrm{~Hz}), 4.09-3.96(2 \mathrm{H}, \mathrm{m}), 2.72(1 \mathrm{H}, \mathrm{sbr}), 2.70-2.61(2 \mathrm{H}, \mathrm{m}) ;{ }^{13} \mathbf{C}-\mathbf{N M R}(125 \mathrm{MHz}$, $\mathrm{CDCl}_{3}$ ): 200.7, 160.4, 138.5, 134.5, 130.4, 125.3, 120.3, 118.2, 117.2, 109.2, 89.3, 44.4, 39.9; HRMS (ESI) calcd for $\mathrm{C}_{14} \mathrm{H}_{15} \mathrm{NKO}_{2}[\mathrm{M}+\mathrm{K}]^{+}: 268.0740$; Found: 268.0742.

2-Allyl-2-hydroxy-1-(4-methoxybenzyl)indolin-3-one (3s): Using the general procedure GP-1, compound 1e (200 mg, $0.74 \mathrm{mmol})$ and allylmagnesium bromide $2 \mathrm{c}(2.2 \mathrm{~mL}, 2.23 \mathrm{mmol})$<smiles>C=CCC1(O)C(=O)c2ccccc2N1[18OH]</smiles>
provided compound $3 \mathbf{s}$ (180 $\mathrm{mg}, 78 \%)$ as a yellow liquid; ${ }^{1} \mathbf{H}-\mathbf{N M R}(500$ $\left.\mathrm{MHz}, \mathrm{CDCl}_{3}\right): 7.55(1 \mathrm{H}, \mathrm{d}, J=7.6 \mathrm{~Hz}), 7.35(1 \mathrm{H}, \mathrm{t}, J=7.6 \mathrm{~Hz}), 7.28(2 \mathrm{H}, \mathrm{t}$, $J=8.4 \mathrm{~Hz}), 6.86(2 \mathrm{H}, \mathrm{d}, J=8.4 \mathrm{~Hz}), 6.71(1 \mathrm{H}, \mathrm{t}, J=7.6 \mathrm{~Hz}), 6.51(1 \mathrm{H}, \mathrm{d}, J$ $=8.4 \mathrm{~Hz}), 5.59-5.52(1 \mathrm{H}, \mathrm{m}), 5.06(2 \mathrm{H}, \mathrm{dd}, J=17.6,16.8 \mathrm{~Hz}), 4.62-4.49$ $(2 \mathrm{H}, \mathrm{m}), 3.79(3 \mathrm{H}, \mathrm{s}), 3.04(1 \mathrm{H}, \mathrm{sbr}), 2.72-2.63(2 \mathrm{H}, \mathrm{m}) ;{ }^{13} \mathrm{C}-\mathrm{NMR}\left(125 \mathrm{MHz}, \mathrm{CDCl}_{3}\right): 200.9$, 160.6, 159.0, 138.6, 130.3, 129.9, 128.8, 128.4, 125.2, 120.4, 118.3, 114.3, 109.5, 89.5, 55.4, 45.4, 40.3; HRMS (ESI) calcd for $\mathrm{C}_{19} \mathrm{H}_{19} \mathrm{NNaO}_{3}[\mathrm{M}+\mathrm{Na}]^{+}: 332.1263$; Found: 332.1269.

2-Allyl-2-hydroxy-1-phenylindolin-3-one (3t): Using the general procedure GP-1, compound 1f (200 $\mathrm{mg}, 0.89 \mathrm{mmol})$ and allylmagnesium bromide $2 \mathbf{c}(2.7 \mathrm{~mL}, 2.66 \mathrm{mmol})$ provided compound<smiles>C=CCC1(O)C(=O)c2ccccc2N1c1ccccc1</smiles>
3t (182 mg, $77 \%)$ as a yellow liquid; ${ }^{1} \mathbf{H}-\mathbf{N M R}\left(500 \mathrm{MHz}, \mathrm{CDCl}_{3}\right): 7.61$ $(1 \mathrm{H}, \mathrm{d}, J=7.6 \mathrm{~Hz}), 7.52(2 \mathrm{H}, \mathrm{d}, J=8.4 \mathrm{~Hz}), 7.45-7.41(3 \mathrm{H}, \mathrm{m}), 7.29(1 \mathrm{H}, \mathrm{t}$, $J=7.6 \mathrm{~Hz}), 6.92(1 \mathrm{H}, \mathrm{d}, J=8.4 \mathrm{~Hz}), 6.82(1 \mathrm{H}, \mathrm{t}, J=6.7 \mathrm{~Hz}), 5.53-5.44(1 \mathrm{H}$, $\mathrm{m})$, 4.98-4.93 $(2 \mathrm{H}, \mathrm{m}), 3.40(1 \mathrm{H}, \mathrm{sbr}), 2.65-2.61(1 \mathrm{H}, \mathrm{m}), 2.57-2.53(1 \mathrm{H}, \mathrm{m})$; ${ }^{13}$ C-NMR (125 MHz, $\left.\mathrm{CDCl}_{3}\right)$ : 200.6, 159.0, 138.3, 138.2, 129.8, 129.6, 126.6, 126.1, 125.4, 120.6, 119.5, 118.6, 110.6, 90.1, 39.6; HRMS (ESI) calcd for $\mathrm{C}_{17} \mathrm{H}_{16} \mathrm{NO}_{2}[\mathrm{M}+\mathrm{H}]^{+}$: 266.1181; Found: 266.1194. 
2-Allyl-1-benzyl-5-chloro-2-hydroxyindolin-3-one (3u): Using the general procedure GP-1, compound $1 \mathbf{j}$ (200 mg, $0.73 \mathrm{mmol}$ ) and allylmagnesium bromide $2 \mathbf{c}(2.2 \mathrm{~mL}, 2.19 \mathrm{mmol})$<smiles>C=CCC1(O)C(=O)c2cc(Cl)ccc2N1Cc1ccccc1</smiles>
provided compound 3u (147 mg, 64\%) as a brown liquid; ${ }^{1} \mathbf{H}-\mathbf{N M R}$ $\left(400 \mathrm{MHz}, \mathrm{CDCl}_{3}\right): 7.50(1 \mathrm{H}, \mathrm{d}, J=2.0 \mathrm{~Hz}), 7.35-7.27(6 \mathrm{H}, \mathrm{m}), 6.42$ $(1 \mathrm{H}, \mathrm{d}, J=8.8 \mathrm{~Hz}), 5.62-5.51(1 \mathrm{H}, \mathrm{m}), 5.13-5.05(2 \mathrm{H}, \mathrm{m}), 4.60(2 \mathrm{H}, \mathrm{q}, J$ $=16.6 \mathrm{~Hz}), 2.69-2.66(2 \mathrm{H}, \mathrm{m}) ;{ }^{13} \mathrm{C}-\mathrm{NMR}\left(100 \mathrm{MHz}, \mathrm{CDCl}_{3}\right): 199.8$, 158.8, 138.2, 137.5, 129.9, 129.0, 127.6, 127.1, 124.5, 123.7, 120.9, 119.4, 110.7, 90.0, 46.0, 40.3; HRMS (ESI) calcd for $\mathrm{C}_{18} \mathrm{H}_{16} \mathrm{ClNNaO}_{2}[\mathrm{M}+\mathrm{Na}]^{+}: 336.0767$; Found: 336.0778 .

2-Hydroxy-2-(4-methoxyphenyl)-1-methylindolin-3-one (3v): Using the general procedure GP1, compound 1a (200 mg, $1.23 \mathrm{mmol}$ ) and $p$-methoxyphenylmagnesium bromide $\mathbf{2 b}(4.9 \mathrm{~mL}$,

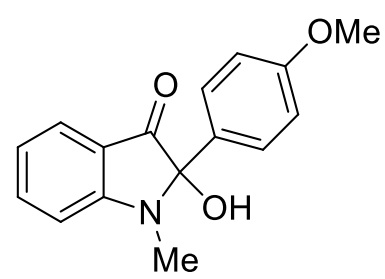
$4.92 \mathrm{mmol}$ ) provided compound $\mathbf{3 v}(217 \mathrm{mg}, 65 \%)$ as a deep green liquid; ${ }^{1} \mathbf{H}-\mathbf{N M R}\left(500 \mathrm{MHz}, \mathrm{CDCl}_{3}\right):$ 7.52-7.49 $(2 \mathrm{H}, \mathrm{m}), 7.32-7.30(2 \mathrm{H}$, m), 6.87-6.85 (2H, m), 6.73-6.71 (2H, m), $3.77(3 \mathrm{H}, \mathrm{d}, J=1.7 \mathrm{~Hz})$, $3.63(1 \mathrm{H}, \mathrm{sbr}), 2.82(3 \mathrm{H}, \mathrm{d}, J=1.7 \mathrm{~Hz}) ;{ }^{13} \mathbf{C}-\mathbf{N M R}\left(100 \mathrm{MHz}, \mathrm{CDCl}_{3}\right)$ : $200.2,161.1,160.1,138.8,127.9,127.6,126.1,118.0,117.4,114.3$, 108.0, 90.8, 55.4, 27.5; HRMS (ESI) calcd for $\mathrm{C}_{16} \mathrm{H}_{16} \mathrm{NO}_{3}[\mathrm{M}+\mathrm{H}]^{+}$: 270.1130; Found: 270.1135 .

1-Benzyl-2-hydroxy-2-(4-methoxyphenyl)indolin-3-one (3w): Using the general procedure GP1, compound 1d (200 mg, $0.84 \mathrm{mmol})$ and $p$-methoxyphenylmagnesium bromide $\mathbf{2 b}(3.3 \mathrm{~mL}$,

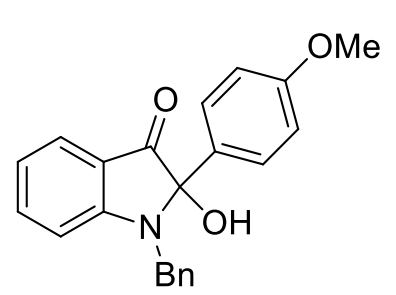

$3.36 \mathrm{mmol})$ provided compound $\mathbf{3 w}(182 \mathrm{mg}, 62 \%)$ as a yellow liquid; ${ }^{1}$ H-NMR $\left(500 \mathrm{MHz}, \mathrm{CDCl}_{3}\right): 7.89(1 \mathrm{H}, \mathrm{d}, J=8.4 \mathrm{~Hz}), 7.52(1 \mathrm{H}, \mathrm{d}, J=$ 7.6 Hz), 7.38-7.31 (4H, m), 7.24-7.19 (2H, m), $6.92(1 \mathrm{H}, \mathrm{d}, J=9.2 \mathrm{~Hz})$, $6.83(2 \mathrm{H}, \mathrm{d}, J=8.4 \mathrm{~Hz}), 6.71(1 \mathrm{H}, \mathrm{dd}, J=7.6,7.6 \mathrm{~Hz}), 6.51(1 \mathrm{H}, \mathrm{d}, J=$ $8.4 \mathrm{~Hz}), 4.36(2 \mathrm{H}, \mathrm{s}), 3.75(3 \mathrm{H}, \mathrm{s}), 3.38(1 \mathrm{H}, \mathrm{sbr}) ;{ }^{13} \mathrm{C}-\mathbf{N M R}(100 \mathrm{MHz}$,

$\left.\mathrm{CDCl}_{3}\right): 200.0,160.7,160.3,138.7,138.0,132.6,128.9,128.8,127.6,127.2,126.1,111.6$, 114.4, 109.1, 91.6, 55.4, 47.2; HRMS (ESI) calcd for $\mathrm{C}_{22} \mathrm{H}_{20} \mathrm{NO}_{3}[\mathrm{M}+\mathrm{H}]^{+}$: 346.1443; Found: 346.1447. 
1-Allyl-2-hydroxy-5-methoxy-2-(4-methoxyphenyl)indolin-3-one (3x): Using the general procedure GP-1, compound $\mathbf{1 v}(200 \mathrm{mg}, 0.91 \mathrm{mmol})$ and $p$-methoxyphenylmagnesium bromide

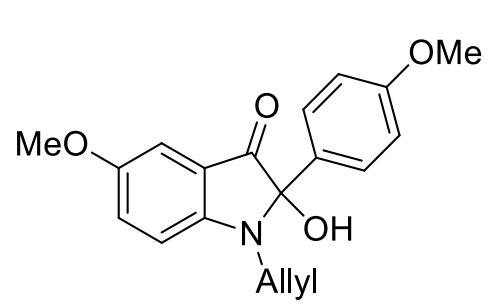

2b (3.6 mL, $3.64 \mathrm{mmol})$ provided compound 3x (184 mg, 62\%) as a yellowish green liquid; ${ }^{1} \mathbf{H}-\mathbf{N M R}\left(500 \mathrm{MHz}, \mathrm{CDCl}_{3}\right): 7.33$ $(2 \mathrm{H}, \mathrm{d}, J=8.4 \mathrm{~Hz}), 7.16(1 \mathrm{H}, \mathrm{dd}, J=5.9,2.5 \mathrm{~Hz}), 7.0(1 \mathrm{H}, \mathrm{d}, J=$ $2.5 \mathrm{~Hz}), 6.85(2 \mathrm{H}, \mathrm{d}, J=8.4 \mathrm{~Hz}), 6.73(1 \mathrm{H}, \mathrm{d}, J=8.4 \mathrm{~Hz}), 5.84-$ $5.77(1 \mathrm{H}, \mathrm{m}), 5.23(1 \mathrm{H}, \mathrm{d}, J=16.8 \mathrm{~Hz}), 5.13(1 \mathrm{H}, \mathrm{d}, J=10.1$ $\mathrm{Hz}), 3.87-3.81(2 \mathrm{H}, \mathrm{m}), 3.78(3 \mathrm{H}, \mathrm{s}), 3.74(3 \mathrm{H}, \mathrm{s}) ;{ }^{13} \mathbf{C}-\mathbf{N M R}\left(125 \mathrm{MHz}, \mathrm{CDCl}_{3}\right): 200.2,160.2$, 156.7, 152.9, 134.9, 128.6, 128.5, 127.7, 117.4, 116.9, 114.3, 110.4, 106.8, 91.8, 56.1, 55.4, 45.7; HRMS (ESI) calcd for $\mathrm{C}_{19} \mathrm{H}_{20} \mathrm{NO}_{4}[\mathrm{M}+\mathrm{H}]^{+}: 326.1392$; Found: 326.1395.

2-Benzyl-2-hydroxy-1-methylindolin-3-one (3y): Using the general procedure GP-1, compound 1a (200 mg, $1.23 \mathrm{mmol})$ and benzylmagnesium bromide $2 \mathrm{~d}(4.9 \mathrm{~mL}, 4.92 \mathrm{mmol})$ provided<smiles>CN1c2ccccc2C(=O)C1(O)Cc1ccccc1</smiles>
compound 3y (232 $\mathrm{mg}, 74 \%)$ as a deep brown liquid; ${ }^{1} \mathbf{H}-\mathbf{N M R}(500$ $\left.\mathrm{MHz}, \mathrm{CDCl}_{3}\right): 7.41(1 \mathrm{H}, \mathrm{d}, J=7.5 \mathrm{~Hz}), 7.36(1 \mathrm{H}, \mathrm{t}, J=8.4 \mathrm{~Hz}), 7.17-$ $7.09(5 \mathrm{H}, \mathrm{m}), 6.60(1 \mathrm{H}, \mathrm{t}, J=7.5 \mathrm{~Hz}), 6.55(1 \mathrm{H}, \mathrm{d}, J=8.4 \mathrm{~Hz}), 3.21$ $(1 \mathrm{H}, \mathrm{d}, J=13.4 \mathrm{~Hz}), 3.12(1 \mathrm{H}, \mathrm{d}, J=17.6 \mathrm{~Hz}), 2.97(3 \mathrm{H}, \mathrm{s}) ;{ }^{13} \mathbf{C}-\mathbf{N M R}$ (125 MHz, $\left.\mathrm{CDCl}_{3}\right): 201.3,161.0,138.6,134.0,130.2,128.3,127.0,125.2,118.1,117.7,108.2$, 89.9, 40.9, 27.5, ; HRMS (ESI) calcd for $\mathrm{C}_{16} \mathrm{H}_{16} \mathrm{NO}_{2}[\mathrm{M}+\mathrm{H}]^{+}$: 254.1181; Found: 254.1184.

2-Benzyl-2-hydroxy-1-(4-methoxybenzyl)indolin-3-one (3z): Using the general procedure GP-1, compound 1e (200 mg, $0.74 \mathrm{mmol})$ and benzylmagnesium bromide $2 \mathbf{d}(3.0 \mathrm{~mL}, 2.96 \mathrm{mmol})$<smiles>O=C1c2ccccc2N([18OH])C1(O)Cc1ccccc1</smiles>
provided compound $\mathbf{3 z}$ (202 $\mathrm{mg}, 76 \%$ ) as a deep brown liquid; ${ }^{1} \mathbf{H}-\mathbf{N M R}$ $\left(500 \mathrm{MHz}, \mathrm{CDCl}_{3}\right): 7.46(1 \mathrm{H}, \mathrm{d}, J=7.6 \mathrm{~Hz}), 7.30(1 \mathrm{H}, \mathrm{t}, J=7.5 \mathrm{~Hz})$, $7.22(2 \mathrm{H}, \mathrm{d}, J=8.4 \mathrm{~Hz}), 7.15-7.08(4 \mathrm{H}, \mathrm{m}), 6.90-6.82(3 \mathrm{H}, \mathrm{m}), 6.60$ $(1 \mathrm{H}, \mathrm{t}, J=7.6 \mathrm{~Hz}), 6.34(1 \mathrm{H}, \mathrm{d}, J=8.4 \mathrm{~Hz}), 4.62(1 \mathrm{H}, \mathrm{d}, J=7.6 \mathrm{~Hz})$, $4.47(1 \mathrm{H}, \mathrm{d}, J=15.9 \mathrm{~Hz}), 3.81(1 \mathrm{H}, \mathrm{s}), 3.78(3 \mathrm{H}, \mathrm{s}), 3.29(1 \mathrm{H}, \mathrm{d}, J=14.2 \mathrm{~Hz}), 3.17(1 \mathrm{H}, \mathrm{d}, J=$ $14.2 \mathrm{~Hz}) ;{ }^{13} \mathrm{C}-\mathrm{NMR}\left(125 \mathrm{MHz}, \mathrm{CDCl}_{3}\right): 201.2,160.3,158.9,138.3,133.8,130.3,129.7,128.8$, 128.5, 128.2, 127.1, 125.1, 118.4, 118.0, 114.1, 109.5, 90.4, 55.4, 45.7, 41.9; HRMS (ESI) calcd for $\mathrm{C}_{23} \mathrm{H}_{22} \mathrm{NO}_{3}[\mathrm{M}+\mathrm{H}]^{+}:$360.1600; Found: 360.1614 . 
1-Allyl-2-hydroxy-2-methylindolin-3-one (3a'): Using the general procedure GP-1, compound 1c $(200 \mathrm{mg}, 1.05 \mathrm{mmol})$ and methylmagnesium bromide $2 \mathbf{e}(4.2 \mathrm{~mL}, 4.20 \mathrm{mmol})$ provided<smiles>CC1(O)C(=O)c2ccccc2N1[AlH2]</smiles>
compound 3a' (155 mg, 72\%) as a yellow liquid; ${ }^{\mathbf{1}} \mathbf{H}-\mathbf{N M R}\left(400 \mathrm{MHz}, \mathrm{CDCl}_{3}\right)$ : $7.56(1 \mathrm{H}, \mathrm{d}, J=7.3 \mathrm{~Hz}), 7.47-7.43(1 \mathrm{H}, \mathrm{m}), 6.74(1 \mathrm{H}, \mathrm{t}, J=7.3 \mathrm{~Hz}), 6.67(1 \mathrm{H}$, $\mathrm{d}, J=8.6 \mathrm{~Hz}), 5.94-5.86(1 \mathrm{H}, \mathrm{m}), 5.29-5.20(2 \mathrm{H}, \mathrm{m}), 4.10-3.94(2 \mathrm{H}, \mathrm{m}), 1.46$ $(3 \mathrm{H}, \mathrm{s}) ;{ }^{13} \mathrm{C}-\mathrm{NMR}\left(125 \mathrm{MHz}, \mathrm{CDCl}_{3}\right): 200.9,159.5,138.6,134.3,125.6$, 118.1, 117.4, 117.0, 109.3, 88.2, 44.0, 21.2; HRMS (ESI) calcd for $\mathrm{C}_{12} \mathrm{H}_{14} \mathrm{NO}_{2}[\mathrm{M}+\mathrm{H}]^{+}$: 204.1025; Found: 204.1026.

1-Benzyl-2-hydroxy-2,5-dimethylindolin-3-one (3b'): Using the general procedure GP-1, compound 1t (200 mg, $0.79 \mathrm{mmol})$ and methylmagnesium bromide 2e $(3.2 \mathrm{~mL}, 3.16 \mathrm{mmol})$<smiles>Cc1ccc2c(c1)C(=O)C(C)(O)N2Cc1ccccc1</smiles>
provided compound 3b' (148 mg, 70\%) as a yellow liquid; ${ }^{1} \mathbf{H}-\mathbf{N M R}(500$ $\left.\mathrm{MHz}, \mathrm{CDCl}_{3}\right): 7.38(1 \mathrm{H}, \mathrm{s}), 7.34-7.24(5 \mathrm{H}, \mathrm{m}), 7.19(1 \mathrm{H}, \mathrm{dd}, J=7.4,1.0$ $\mathrm{Hz}), 6.41(1 \mathrm{H}, \mathrm{d}, J=8.3 \mathrm{~Hz}), 4.57(2 \mathrm{H}, \mathrm{s}), 3.01(1 \mathrm{H}, \mathrm{sbr}), 2.23(3 \mathrm{H}, \mathrm{s})$, $1.46(3 \mathrm{H}, \mathrm{s}) ;{ }^{13} \mathrm{C}-\mathbf{N M R}\left(100 \mathrm{MHz}, \mathrm{CDCl}_{3}\right): 201.1,158.3,139.9,138.4$, $128.9,127.9,127.4,126.9,126.6,126.3,125.1,123.2,117.6,109.4,88.6,45.6,21.5,20.5$; HRMS (ESI) calcd for $\mathrm{C}_{17} \mathrm{H}_{18} \mathrm{NO}_{2}[\mathrm{M}+\mathrm{H}]^{+}:$268.1338; Found: 268.1331.

\subsubsection{General procedure for the synthesis of 2 -hydroxyindoxyls $3 c^{\prime}-3 g^{\prime}$ (GP-2):}

To a stirring solution of $N$-substituted 3-hydroxy-2-oxindoles 1 (1 equiv.) in dry THF, Grignard reagent solution (4 equiv.; $1 \mathrm{M}$ in THF) was added dropwise at room temperature. The reaction mixture was then refluxed at $70{ }^{\circ} \mathrm{C}$ for $6 \mathrm{~h}$. After completion of reaction as monitored by the TLC, the reaction mixture was cooled to room temperature and then quenched with saturated aqueous $\mathrm{NH}_{4} \mathrm{Cl}$ solution followed by extraction with EtOAc $(3 \times 20 \mathrm{~mL})$. The combined organic phases were washed with brine, dried over anhydrous $\mathrm{Na}_{2} \mathrm{SO}_{4}$, filtered and concentrated in vacuo. The crude residue was then purified by column chromatography on silica gel with ethylacetate-hexane (10/90 to 20/80) to give compounds $\mathbf{3} \mathbf{c}^{\prime} \mathbf{- 3} \mathbf{g}^{\prime}$. 
2-Ethyl-2-hydroxy-1-methylindolin-3-one (3c'): Using the general procedure GP-2, compound $1 \mathbf{a}(200 \mathrm{mg}, 1.23 \mathrm{mmol})$ and ethylmagnesium bromide $2 \mathrm{ff}(4.9 \mathrm{~mL}, 4.92 \mathrm{mmol})$ provided<smiles>CCC1(O)C(=O)c2ccccc2N1C</smiles>
compound 3c' (138 mg, 58\%) as a yellow liquid; ${ }^{\mathbf{1}} \mathbf{H}-\mathbf{N M R}\left(400 \mathrm{MHz}, \mathrm{CDCl}_{3}\right)$ : $7.53(1 \mathrm{H}, \mathrm{d}, J=7.8 \mathrm{~Hz}), 7.47(1 \mathrm{H}, \mathrm{t}, J=7.8 \mathrm{~Hz}), 6.72-6.68(2 \mathrm{H}, \mathrm{m}), 2.96(3 \mathrm{H}$, s), 2.05-1.96 $(1 \mathrm{H}, \mathrm{m}), 1.93-1.84(1 \mathrm{H}, \mathrm{m}), 1.67(1 \mathrm{H}, \mathrm{sbr}), 0.68(3 \mathrm{H}, \mathrm{t}, J=7.4$ $\mathrm{Hz}) ;{ }^{13} \mathbf{C}-\mathbf{N M R}\left(125 \mathrm{MHz}, \mathrm{CDCl}_{3}\right): 201.5,161.0,138.7,135.7,125.2,117.7$, 108.1, 90.5, 27.8, 26.6, 7.7; HRMS (ESI) calcd for $\mathrm{C}_{11} \mathrm{H}_{13} \mathrm{NKO}_{2}[\mathrm{M}+\mathrm{K}]^{+}$: 230.0583; Found: 230.0597.

1,2-Diethyl-2-hydroxyindolin-3-one (3d'): Using the general procedure GP-2, compound $\mathbf{1 b}$ (200 mg, $1.13 \mathrm{mmol}$ ) and ethylmagnesium bromide $\mathbf{2 f}(4.5 \mathrm{~mL}, 4.52 \mathrm{mmol}$ ) provided compound<smiles>CCN1c2ccccc2C(=O)C1(O)CC</smiles>
3d' (130 mg, 56\%) as a yellow liquid; ${ }^{1} \mathbf{H}-\mathbf{N M R}\left(400 \mathrm{MHz}, \mathrm{CDCl}_{3}\right): 7.52(1 \mathrm{H}$, $\mathrm{d}, J=7.8 \mathrm{~Hz}), 7.46(1 \mathrm{H}, \mathrm{t}, J=7.8 \mathrm{~Hz}), 6.71-6.67(2 \mathrm{H}, \mathrm{m}), 3.55-3.46(1 \mathrm{H}, \mathrm{m})$, 3.42-3.33 (1H, m), 2.63 (1H, sbr), 2.05-1.96 (1H, m), 1.93-1.85 (1H, m), 1.29 $(3 \mathrm{H}, \mathrm{t}, J=7.3 \mathrm{~Hz}), 0.69(3 \mathrm{H}, \mathrm{t}, J=7.8 \mathrm{~Hz}) ;{ }^{13} \mathbf{C}-\mathbf{N M R}\left(125 \mathrm{MHz}, \mathrm{CDCl}_{3}\right)$ : 201.8, 160.5, 138.7, 125.4, 118.2, 117.4,108.2, 90.9, 35.8, 28.3, 14.6, 7.7; HRMS (ESI) calcd for $\mathrm{C}_{12} \mathrm{H}_{15} \mathrm{NNaO}_{2}[\mathrm{M}+\mathrm{Na}]^{+}:$228.1000; Found: 228.1017.

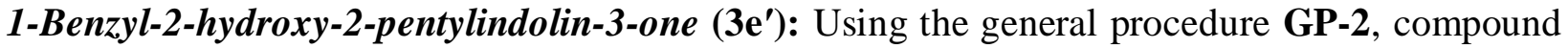
1d (200 mg, $0.84 \mathrm{mmol})$ and pentylmagnesium bromide $\mathbf{2 g}(3.4 \mathrm{~mL}, 3.36 \mathrm{mmol})$ provided<smiles>CCCCCC1(O)C(=O)c2ccccc2N1Cc1ccccc1</smiles>
compound 3e' (152 mg, 58\%) as a yellow liquid; ${ }^{1} \mathbf{H}-\mathbf{N M R}(400 \mathrm{MHz}$, $\left.\mathrm{CDCl}_{3}\right): 7.56(1 \mathrm{H}, \mathrm{d}, J=7.8 \mathrm{~Hz}), 7.38-7.29(6 \mathrm{H}, \mathrm{m}), 6.73(1 \mathrm{H}, \mathrm{t}, J=$ $7.3 \mathrm{~Hz}), 6.55(1 \mathrm{H}, \mathrm{d}, J=8.3 \mathrm{~Hz}), 4.58(2 \mathrm{H}, \mathrm{q}, J=16.6 \mathrm{~Hz}), 2.79(1 \mathrm{H}$, Sbr), 1.98-1.92 (1H, m), 1.87-1.80 (1H, m), 1.11-0.99 (4H, m), 0.90$0.83(2 \mathrm{H}, \mathrm{m}), 0.78(3 \mathrm{H}, \mathrm{t}, J=6.4 \mathrm{~Hz}) ;{ }^{13} \mathbf{C}-\mathbf{N M R}\left(100 \mathrm{MHz}, \mathrm{CDCl}_{3}\right): 201.4,160.8,138.5,128.9$, $128.0,127.5,127.4,127.2$, 125.2, 118.2, 109.2, 90.5, 45.8, 35.7, 31.8, 22.8, 22.4, 14.0; HRMS (ESI) calcd for $\mathrm{C}_{20} \mathrm{H}_{23} \mathrm{NNaO}_{2}[\mathrm{M}+\mathrm{Na}]^{+}$: 332.1627; Found: 332.1625. 
2-Hydroxy-1-(4-methoxybenzyl)-2-(octan-3-yl)indolin-3-one (3f'): Using the general procedure GP-2, compound 1e (200 mg, $0.74 \mathrm{mmol})$ and 2-(ethyl)hexylmagnesium bromide $2 \mathbf{h}(3.0 \mathrm{~mL}$,

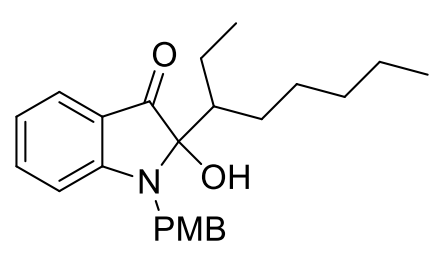

$2.96 \mathrm{mmol}$ ) provided compound $\mathbf{3 f ^ { \prime }}(172 \mathrm{mg}, 60 \%$ ) as a yellow liquid; ${ }^{1} \mathbf{H}$-NMR (400 MHz, $\left.\mathrm{CDCl}_{3}\right): 7.50(1 \mathrm{H}, \mathrm{d}, J=7.8 \mathrm{~Hz}), 7.35$ $(1 \mathrm{H}, \mathrm{t}, J=8.3 \mathrm{~Hz}), 7.28(2 \mathrm{H}, \mathrm{d}, J=8.3 \mathrm{~Hz}), 6.85(2 \mathrm{H}, \mathrm{d}, J=8.8$ $\mathrm{Hz}), 6.71(1 \mathrm{H}, \mathrm{t}, J=6.4 \mathrm{~Hz}), 6.50(1 \mathrm{H}, \mathrm{dd}, J=5.4,3.0 \mathrm{~Hz}), 4.57$

$(1 \mathrm{H}, \mathrm{d}, J=17.0 \mathrm{~Hz}), 4.42(1 \mathrm{H}, \mathrm{d}, J=16.1 \mathrm{~Hz}), 3.79(3 \mathrm{H}, \mathrm{s}), 2.07-1.82(2 \mathrm{H}, \mathrm{m}), 1.75-1.58(3 \mathrm{H}$, m), 1.13-1.04 (3H, m), 0.89-0.81 (5H, m), 0.78-0.74 (3H, m), $0.70(1 \mathrm{H}, \mathrm{t}, J=7.3 \mathrm{~Hz}) ;{ }^{13} \mathbf{C}-\mathbf{N M R}$ (100 MHz, $\left.\mathrm{CDCl}_{3}\right): 201.3,160.3,159.0,138.4,129.9,128.6,127.6,125.3,118.1,114.2,109.4$, 90.6, 55.4, 45.5, 40.1, 31.8, 28.2, 26.2, 23.1, 22.8, 10.8, 9.9; HRMS (ESI) calcd for $\mathrm{C}_{24} \mathrm{H}_{31} \mathrm{NNaO}_{3}[\mathrm{M}+\mathrm{Na}]^{+}: 404.2202$; Found: 404.2203.

1-Benzyl-2-cyclohexyl-2-hydroxyindolin-3-one $\left(\mathbf{3 g}^{\prime}\right)$ : Using the general procedure GP-2, compound 1d (200 mg, $0.84 \mathrm{mmol})$ and cyclohexylmagnesium bromide $2 \mathbf{i}(3.4 \mathrm{~mL}, 3.36 \mathrm{mmol})$<smiles>O=C1c2ccccc2N(Cc2ccccc2)C1(O)C1CCCCC1</smiles>
provided compound $\mathbf{3 g}$ ' (174 $\mathrm{mg}, 64 \%)$ as a yellow liquid; ${ }^{\mathbf{1}} \mathbf{H}$-NMR (400 $\left.\mathrm{MHz}, \mathrm{CDCl}_{3}\right): 7.61(1 \mathrm{H}, \mathrm{d}, J=7.3 \mathrm{~Hz}), 7.46-7.32(6 \mathrm{H}, \mathrm{m}), 6.84(1 \mathrm{H}, \mathrm{dd}, J$ = 9.7, $7.3 \mathrm{~Hz}), 6.57(1 \mathrm{H}, \mathrm{d}, J=8.3 \mathrm{~Hz}), 4.67(2 \mathrm{H}, \mathrm{s}), 3.71(1 \mathrm{H}, \mathrm{sbr}), 2.04-$ $1.97(2 \mathrm{H}, \mathrm{m}), 1.87-1.59(6 \mathrm{H}, \mathrm{m}), 1.17-1.09(1 \mathrm{H}, \mathrm{m}), 1.00-0.92(2 \mathrm{H}, \mathrm{m})$; ${ }^{13}$ C-NMR (100 MHz, $\left.\mathrm{CDCl}_{3}\right): 202.2,161.2,138.3,128.8,127.3,129.0,127.5,127.2,124.8$, 118.2, 109.5, 91.9, 46.4, 35.7, 27.0, 26.5, 26.2, 25.3, 24.3; HRMS (ESI) calcd for $\mathrm{C}_{21} \mathrm{H}_{23} \mathrm{NNaO}_{2}$ $[\mathrm{M}+\mathrm{Na}]^{+}:$344.1626; Found: 344.1628 . 


\subsection{Gram scale experiment:}

\section{Preparation of 5-fluoro-2-hydroxy-1-methyl-2-phenylindolin-3-one 3g:}

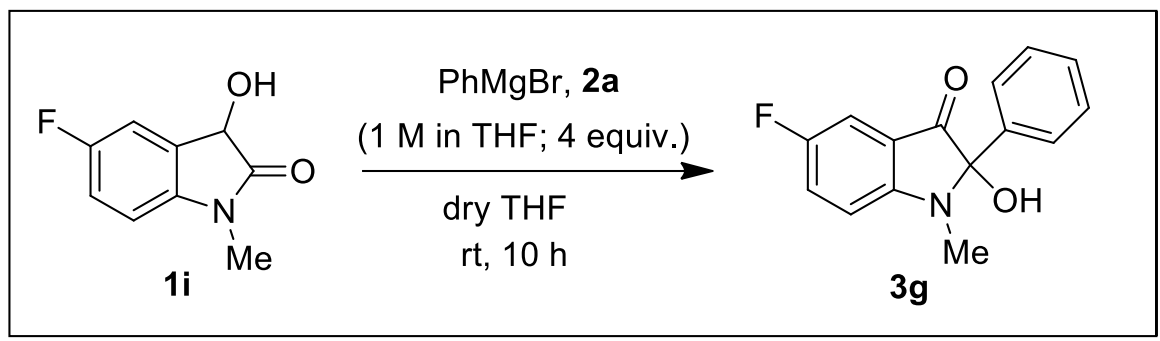

Scheme S2. Gram scale synthesis of $\mathbf{3 g}$.

To a stirring solution of $\mathbf{1 i}(1.0 \mathrm{~g}, 5.52 \mathrm{mmol}, 1$ equiv. $)$ in dry THF $(20 \mathrm{~mL})$, phenylmagnesium bromide 2a (22 mL, $22.08 \mathrm{mmol}, 4$ equiv.) was added dropwise at room temperature. The resulting reaction mixture was then stirred at room temperature for $10 \mathrm{~h}$. After completion of the reaction as monitored by TLC, the mixture was quenched with saturated aqueous $\mathrm{NH}_{4} \mathrm{Cl}$ solution and extracted with EtOAc $(3 \times 20 \mathrm{~mL})$. The combined organic phases were washed with brine, dried over anhydrous $\mathrm{Na}_{2} \mathrm{SO}_{4}$, filtered and concentrated in vacuo. The crude residue was then purified by column chromatography on silica gel with EtOAc-hexane (10/90 to 20/80) to give compound $\mathbf{3 g}(1.10 \mathrm{~g}, 70 \%)$ as a red solid.

\section{Preparation of 1,2-Diallyl-2-hydroxyindolin-3-one 3r:}

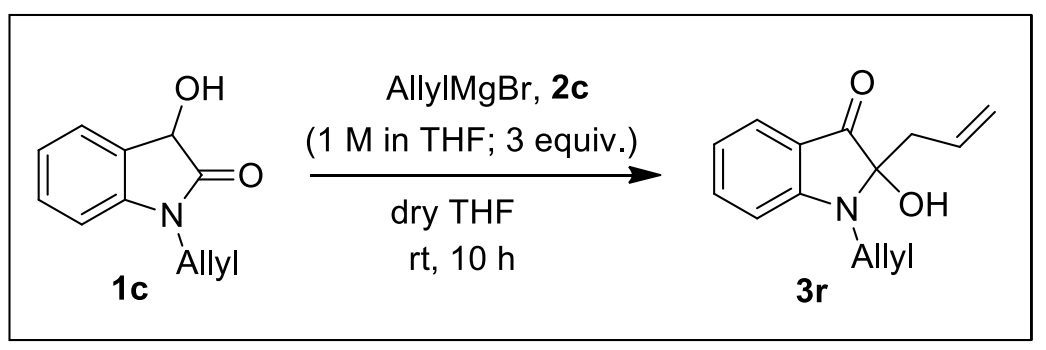

Scheme S3. Gram scale synthesis of 3r.

To a stirring solution of $1 \mathrm{c}(1.0 \mathrm{~g}, 5.28 \mathrm{mmol}, 1$ equiv.) in dry THF $(20 \mathrm{~mL})$, allyllmagnesium bromide $2 \mathrm{c}$ (17 mL, $16.84 \mathrm{mmol}, 3$ equiv) was added dropwise at room temperature. The resulting reaction mixture was then stirred at room temperature for $10 \mathrm{~h}$. After completion of reaction as monitored by TLC, the mixture was quenched with saturated aqueous 
$\mathrm{NH}_{4} \mathrm{Cl}$ solution and extracted with EtOAc $(3 \times 20 \mathrm{~mL})$. The combined organic phases were washed with brine, dried over anhydrous $\mathrm{Na}_{2} \mathrm{SO}_{4}$, filtered and concentrated in vacuo. The crude residue was then purified by column chromatography on silica gel with EtOAc-hexane (10/90 to 20/80) to give compound $\mathbf{3 r}(870 \mathrm{mg}, 72 \%)$ as a yellow liquid.

\subsection{Deuterium labelling experiment:}

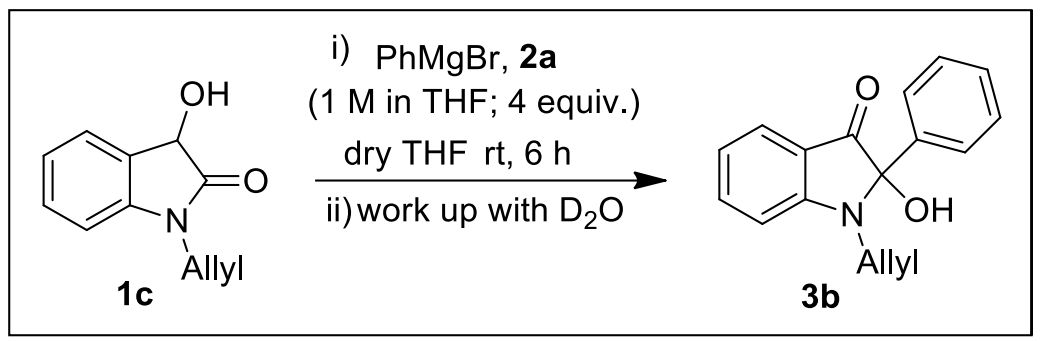

Scheme S4. Deuterium labelling experiment for the synthesis of $\mathbf{3 b}$.

To a stirring solution of $1 \mathrm{c}(50 \mathrm{mg}, 0.26 \mathrm{mmol}, 1$ equiv. $)$ in dry THF $(20 \mathrm{~mL})$, phenylmagnesium bromide $\mathbf{2 a}(1.1 \mathrm{~mL}, 1.1 \mathrm{mmol}, 4$ equiv) was added dropwise at room temperature. The resulting reaction mixture was then stirred at room temperature for $6 \mathrm{~h}$. After completion of reaction as monitored by TLC, the mixture was quenched with $\mathrm{D}_{2} \mathrm{O}$ and extracted with EtOAc $(3 \times 5 \mathrm{~mL})$. The combined organic phases were washed with brine, dried over anhydrous $\mathrm{Na}_{2} \mathrm{SO}_{4}$, filtered and concentrated in vacuo. The crude residue was then purified by column chromatography on silica gel with EtOAc-hexane (10/90 to 20/80) to give exclusively compound $\mathbf{3 b}$ as a brown gummy liquid .

\subsection{Radical quenching experiment:}

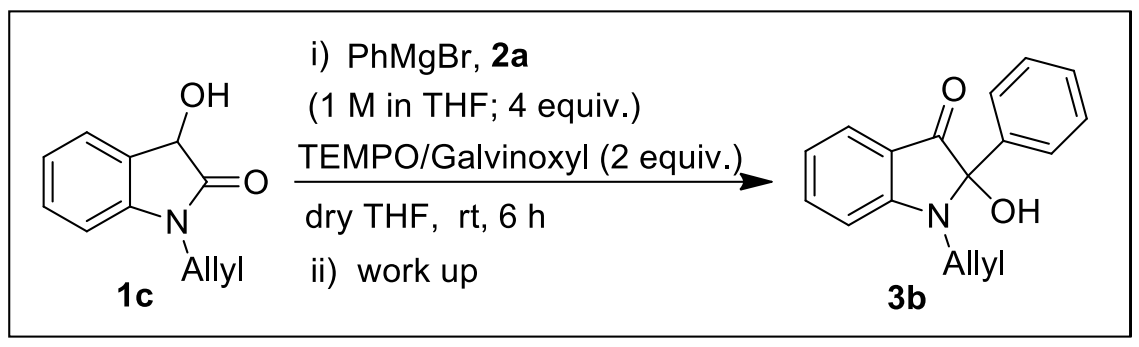

Scheme S5. Deuterium labelling experiment for the synthesis of $\mathbf{3 b}$.

To a stirring solution of $1 \mathrm{c}(50 \mathrm{mg}, 0.26 \mathrm{mmol}, 1$ equiv.) in dry THF (20 mL), phenylmagnesium bromide $\mathbf{2 a}(1.1 \mathrm{~mL}, 1.1 \mathrm{mmol}, 4$ equiv) and radical inhibitor $(0.52 \mathrm{mmol}, 2$ equiv.) were added at room temperature. The resulting reaction mixture was then stirred at room 
temperature for $6 \mathrm{~h}$. After completion of reaction as monitored by TLC, the mixture was quenched with saturated aqueous $\mathrm{NH}_{4} \mathrm{Cl}$ solution and extracted with EtOAc $(3 \times 5 \mathrm{~mL})$. The combined organic phases were washed with brine, dried over anhydrous $\mathrm{Na}_{2} \mathrm{SO}_{4}$, filtered and concentrated in vacuo. The crude residue was then purified by column chromatography on silica gel with EtOAc-hexane (10/90 to 20/80) to give exclusively compound $\mathbf{3 b}$ as a brown gummy liquid (Yield: $76 \%$ in presence of TEMPO and $70 \%$ in presence of Galvinoxyl) .

7.0 General procedure for the synthesis of C2-allyl-2,2-disubstituted oxindoles 6 (GP-3): To a solution of 2-hydroxyindoxyls 3 (1 equiv.) in dry THF ( $5 \mathrm{~mL}$ ) was added allylmagnesium bromide solution (2 equiv., $1 \mathrm{M}$ in THF) dropwise and the resulting mixture was stirred at room temperature for $3 \mathrm{~h}$. After completion of reaction as monitored by the TLC, the reaction mixture was quenched with saturated aqueous $\mathrm{NH}_{4} \mathrm{Cl}$ solution followed by extraction with EtOAc $(3$ x 5 $\mathrm{mL}$ ). The combined organic phases were washed with brine, dried over anhydrous $\mathrm{Na}_{2} \mathrm{SO}_{4}$, filtered and concentrated in vacuo. The crude residue was then purified by column chromatography on silica gel with ethylacetate-hexane (5/95 to 10/90) to give compounds 6a-b (Scheme S6).

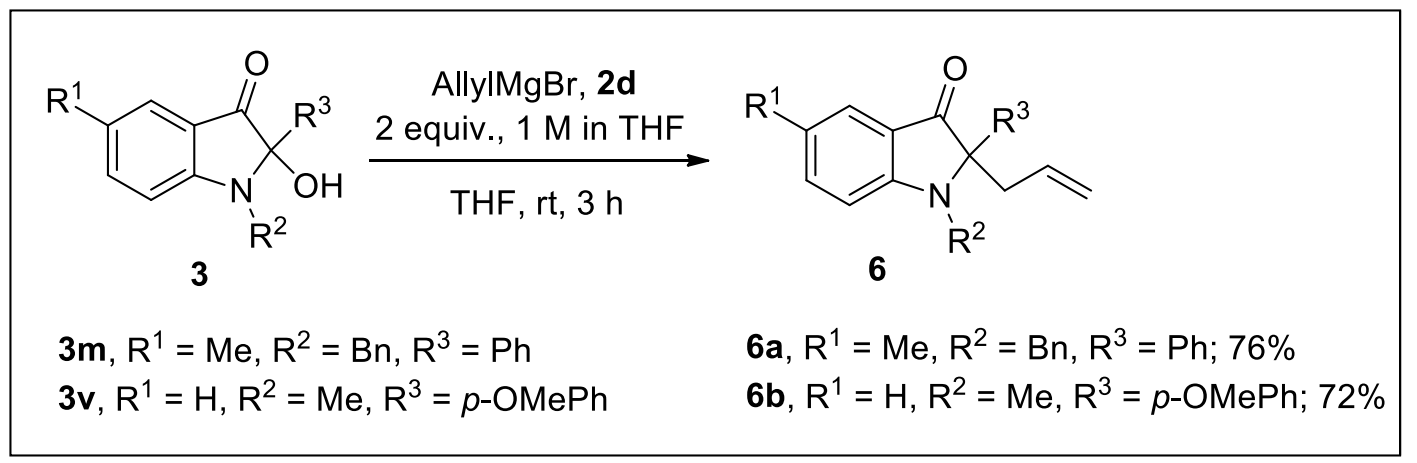

Scheme S6. Synthesis of C2-allyl-2,2-disubstituted oxindoles 6.

2-Allyl-1-benzyl-5-methyl-2-phenylindolin-3-one (6a): Using the general procedure GP-3, compound 3m (100 mg, $0.30 \mathrm{mmol})$ and allylmagnesium bromide $(0.6 \mathrm{~mL}, 0.60 \mathrm{mmol})$ provided<smiles>C=CCC1(c2ccccc2)C(=O)c2cc(C)ccc2N1Cc1ccccc1</smiles>
compound 6a (82 mg, 76\%) as a yellow liquid; ${ }^{1} \mathbf{H}-\mathbf{N M R}(500 \mathrm{MHz}$, $\left.\mathrm{CDCl}_{3}\right): 7.44(1 \mathrm{H}, \mathrm{s}), 7.35-7.20(11 \mathrm{H}, \mathrm{m}), 6.62(1 \mathrm{H}, \mathrm{d}, J=8.2 \mathrm{~Hz})$, 5.59-5.50 (1H, m), $5.00(1 \mathrm{H}, \mathrm{dd}, J=15.2,1.9 \mathrm{~Hz}), 4.93(1 \mathrm{H}, \mathrm{dd}, J=$ 8.8, 1.3 Hz), $4.55(1 \mathrm{H}, \mathrm{d}, J=17.0 \mathrm{~Hz}), 4.30(1 \mathrm{H}, \mathrm{d}, J=16.4 \mathrm{~Hz}), 3.19$ 
$(1 \mathrm{H}, \mathrm{dd}, J=7.6,6.9 \mathrm{~Hz}), 2.96(1 \mathrm{H}, \mathrm{dd}, J=6.9,6.9 \mathrm{~Hz}), 2.31(3 \mathrm{H}, \mathrm{s}) ;{ }^{13} \mathbf{C}-\mathbf{N M R}(75 \mathrm{MHz}$; $\left.\mathrm{CDCl}_{3}\right): 201.5,160.5,139.2,138.0,137.8,131.9,129.1,128.6,128.2,127.4,127.3,126.8$, 124.9, 120.2, 119.6, 109.3, 76.8, 48.6, 38.1, 20.5; HRMS (ESI) calcd for $\mathrm{C}_{25} \mathrm{H}_{24} \mathrm{NO}[\mathrm{M}+\mathrm{H}]^{+}$: 354.1858; Found: 354.1860.

2-Allyl-2-(4-methoxyphenyl)-1-methylindolin-3-one (6b): Using the general procedure GP-3, compound $\mathbf{3 v}$ (100 mg, $0.37 \mathrm{mmol})$ and allylmagnesium bromide $2 \mathbf{2 d}(0.7 \mathrm{~mL}, 0.74 \mathrm{mmol})$

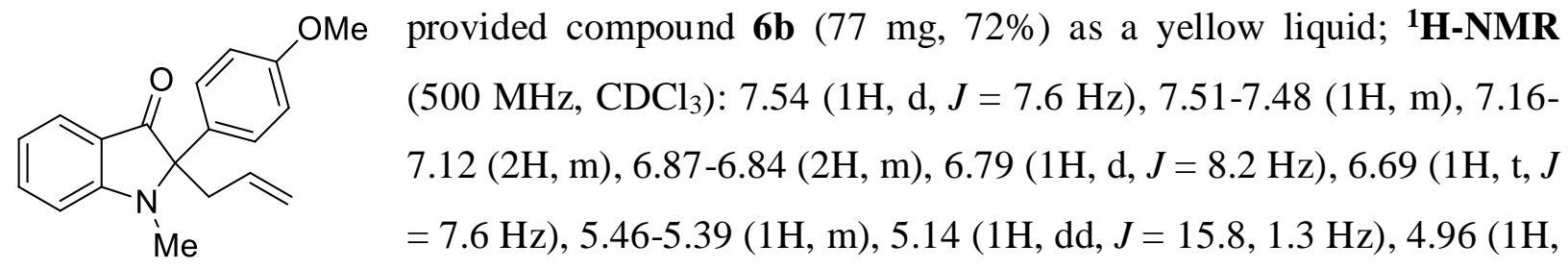
dd, $J=8.2,1.9 \mathrm{~Hz}), 3.77(3 \mathrm{H}, \mathrm{s}), 3.29-3.25(1 \mathrm{H}, \mathrm{m}), 2.93(3 \mathrm{H}, \mathrm{s}), 2.82(1 \mathrm{H}, \mathrm{dd}, J=7.6,6.3 \mathrm{~Hz})$,; ${ }^{13}$ C-NMR (125 MHz; $\left.\mathrm{CDCl}_{3}\right)$ : 201.3, 161.4, 159.4, 137.9, 132.0, 129.2, 127.4, 125.5, 119.1, 119.0, 116.9, 114.5, 107.4, 75.1, 55.4, 38.8, 28.4; HRMS (ESI) calcd for $\mathrm{C}_{19} \mathrm{H}_{20} \mathrm{NO}_{2}[\mathrm{M}+\mathrm{H}]^{+}$: 294.1494; Found: 294.1499.

\subsection{Synthesis of 3-allyl or aryl-3-hydroxy oxindoles $7:^{2}$ \\ 8.0.1 General procedure for the synthesis of 3-allyl-3-hydroxy oxindoles 7a-b from 2- hydroxyindoxyls 3 (GP-4):}

To a solution of 2-allyl-2-hydroxyindoxyls 3 (1 equiv.) in dry DMF (5 mL) was added sodium hydride (2 equiv.) portionwise at $0{ }^{\circ} \mathrm{C}$ and the resulting mixture was stirred at room temperature for $6 \mathrm{~h}$. After completion of reaction as monitored by the TLC, the reaction mixture was quenched with water followed by extraction with EtOAc $(3 \times 5 \mathrm{~mL})$. The combined organic phases were washed with brine, dried over anhydrous $\mathrm{Na}_{2} \mathrm{SO}_{4}$, filtered and concentrated in vacuo. The crude residue was then purified by column chromatography on silica gel with ethylacetate-hexane (10/90 to 20/80) to give compounds 7a-b (Scheme S7). 


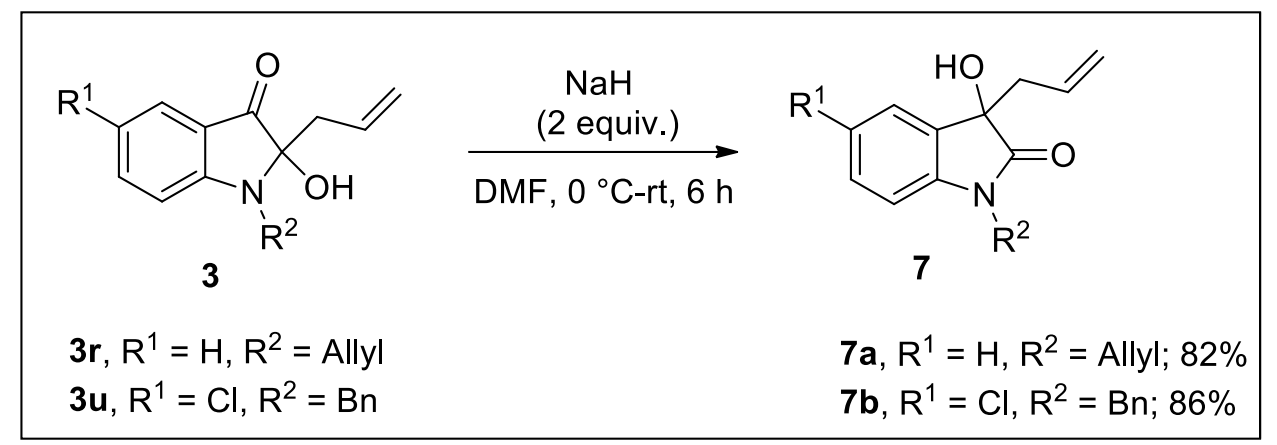

Scheme S7. Synthesis of 3-allyl-3-hydroxy oxindoles 7 from 2-hydroxyindoxyls 3.

1,3-Diallyl-3-hydroxyindolin-2-one (7a): Using the general procedure GP-4, compound 3r (100 $\mathrm{mg}, 0.44 \mathrm{mmol})$ and sodium hydride $(21 \mathrm{mg}, 0.88 \mathrm{mmol})$ provided compound 7a $(82 \mathrm{mg}, 82 \%)$<smiles>C=CCC1(O)C(=O)N([Al])c2ccccc21</smiles>
as a straw yellow solid; ${ }^{1} \mathbf{H}-\mathbf{N M R}\left(400 \mathrm{MHz} \mathrm{CDCl}_{3}\right): 7.39(1 \mathrm{H}, \mathrm{d}, J=7.3$ $\mathrm{Hz}), 7.28-7.24(1 \mathrm{H}, \mathrm{m}), 7.07(1 \mathrm{H}, \mathrm{t}, J=7.9 \mathrm{~Hz}), 6.79(1 \mathrm{H}, \mathrm{d}, J=7.9 \mathrm{~Hz})$, 5.81-5.73 $(1 \mathrm{H}, \mathrm{m}), 5.61-5.50(1 \mathrm{H}, \mathrm{m}), 5.23-5.17(2 \mathrm{H}, \mathrm{m}), 5.04(2 \mathrm{H}, \mathrm{m}), 4.42-$ $4.37(1 \mathrm{H}, \mathrm{m}), 4.18-4.12(1 \mathrm{H}, \mathrm{m}), 4.03(1 \mathrm{H}, \mathrm{s}), 2.78(1 \mathrm{H}, \mathrm{dd}, J=7.3,6.1 \mathrm{~Hz})$, $2.65(1 \mathrm{H}, \mathrm{dd}, J=8.0,4.9 \mathrm{~Hz}) ;{ }^{13} \mathbf{C}-\mathbf{N M R}\left(100 \mathrm{MHz} ; \mathrm{CDCl}_{3}\right): 177.9,142.5,131.2,130.6,129.9$, $129.5,124.2$, 123.1, 120.3, 117.7, 109.3, 76.1, 43.0, 42.4; HRMS (ESI) calcd for $\mathrm{C}_{14} \mathrm{H}_{15} \mathrm{NKO}_{2}$ $[\mathrm{M}+\mathrm{K}]^{+}:$268.0740; Found: 268.0742.

3-Allyl-1-benzyl-5-chloro-3-hydroxyindolin-2-one (7b): Using the general procedure GP-4, compound 3u (100 mg, $0.32 \mathrm{mmol})$ and allylmagnesium bromide 2d (15 mg, $0.64 \mathrm{mmol})$<smiles>C=CCC1(O)C(=O)N(Cc2ccccc2)c2ccc(Cl)cc21</smiles>
provided compound $\mathbf{7 b}(86 \mathrm{mg}, 86 \%)$ as a brown solid; ${ }^{\mathbf{1}} \mathbf{H}-\mathbf{N M R}(500$ $\left.\mathrm{MHz}, \mathrm{CDCl}_{3}\right): 7.37(1 \mathrm{H}, \mathrm{d}, J=1.7 \mathrm{~Hz}), 7.30-7.22(5 \mathrm{H}, \mathrm{m}), 7.13(1 \mathrm{H}$, $\mathrm{dd}, J=5.9,2.6 \mathrm{~Hz}), 6.57(1 \mathrm{H}, \mathrm{d}, J=8.4 \mathrm{~Hz}), 5.60-5.51(1 \mathrm{H}, \mathrm{m}), 5.09$ $(2 \mathrm{H}, \mathrm{dd}, J=15.9,9.3 \mathrm{~Hz}), 4.96(1 \mathrm{H}, \mathrm{d}, J=15.9 \mathrm{~Hz}), 4.67(1 \mathrm{H}, \mathrm{d}, J=$ $15.9 \mathrm{~Hz}), 4.29(1 \mathrm{H}, \mathrm{s}), 2.81(1 \mathrm{H}, \mathrm{dd}, J=6.8,6.7 \mathrm{~Hz}), 2.70(1 \mathrm{H}, \mathrm{dd}, J=8.4,8.4 \mathrm{~Hz})$; ${ }^{13} \mathbf{C}-\mathbf{N M R}$ (125 MHz; $\left.\mathrm{CDCl}_{3}\right):$ 178.0, 140.9, 135.0, 131.6, 130.1, 129.5, 128.9, 128.7, 127.9, 127.3, 124.9, 121.0, 110.6, 76.3, 44.0, 42.9; HRMS (ESI) calcd for $\mathrm{C}_{18} \mathrm{H}_{16} \mathrm{ClNNaO}_{2}[\mathrm{M}+\mathrm{Na}]^{+}$: 336.0767 ; Found: 336.0773. 


\subsubsection{General procedure for the synthesis of 3-aryl or allyl-3-hydroxy oxindoles 7c-e from}

\section{3-hydroxyoxindoles 1 (GP-5):}

To a solution of 3-hydroxyoxindoles 1 (1 equiv.) in dry THF (10 mL) was added aryl or allyl Grignard solution (4 equiv. for aryl and 3 equiv. for allyl Grignard solution; $1 \mathrm{M}$ in THF) portionwise at $0{ }^{\circ} \mathrm{C}$ and the resulting mixture was stirred at room temperature for $6 \mathrm{~h}$. After completion of reaction as monitored by the TLC, the reaction mixture was quenched with aqs. $\mathrm{NH}_{4} \mathrm{Cl}$ followed by extraction with EtOAc $(3 \times 5 \mathrm{~mL})$. The combined organic phases were washed with brine, dried over anhydrous $\mathrm{Na}_{2} \mathrm{SO}_{4}$, filtered and concentrated in vacuo.

The crude residue was then dissolved in $10 \mathrm{~mL}$ of DMF and then sodium hydride (2 equiv.) was added to it portionwise at $0{ }^{\circ} \mathrm{C}$ and the reaction mixture was stirred at room temperature for $6 \mathrm{~h}$. After completion of reaction as monitored by the TLC, the reaction mixture was quenched with water followed by extraction with EtOAc $(3 \times 5 \mathrm{~mL})$. The combined organic phases were washed with brine, dried over anhydrous $\mathrm{Na}_{2} \mathrm{SO}_{4}$, filtered and concentrated in vacuo. The crude residue was then purified by column chromatography on silica gel with ethylacetate-hexane (10/90 to 20/80) to give compounds 7c-e (Scheme S8).

\begin{tabular}{|c|c|c|}
\hline$\sum_{\mathrm{R}^{2}}^{\mathrm{OH}}=\mathrm{ii}$ & $\begin{array}{l}\mathrm{R}^{3} \mathrm{MgBr}(1 \mathrm{M} \text { in } \mathrm{THF}) \\
\mathrm{THF}, \mathrm{rt}, 6 \mathrm{~h} \\
\mathrm{NaH}(2 \text { equiv. }) \\
\mathrm{DMF}, 0{ }^{\circ} \mathrm{C}-\mathrm{rt}, 6 \mathrm{~h}\end{array}$ & $\underbrace{\mathrm{R}_{\mathrm{R}^{2}}}_{7}$ \\
\hline $\begin{array}{l}\text { 1c, } R^{1}=H, R^{2}=\text { Allyl } \\
1 \mathbf{k}, R^{1}=B r, R^{2}=M e \\
1 s, R^{1}=O_{C F}, R^{2}=B n\end{array}$ & & $\begin{array}{l}\text { 7c, } \mathrm{R}^{1}=\mathrm{H}, \mathrm{R}^{2}=\text { Allyl, } \mathrm{R}^{3}=p-\mathrm{OMePh} ; 70 \% \\
7 \mathrm{~d}, \mathrm{R}^{1}=\mathrm{Cl}, \mathrm{R}^{2}=\mathrm{Bn}, \mathrm{R}^{3}=\text { Allyl; } 76 \% \\
7 \mathrm{e}, \mathrm{R}^{1}=\mathrm{OCF}_{3}, \mathrm{R}^{2}=\mathrm{Bn}, \mathrm{R}^{3}=\text { Allyl; } 72 \%\end{array}$ \\
\hline
\end{tabular}

Scheme S8. Synthesis of 3-aryl or allyl-3-hydroxy oxindoles 7 from 3-hydroxyoxindoles 1.

1-Allyl-3-hydroxy-3-(4-methoxyphenyl)indolin-2-one (7c): Using the general procedure GP-5,

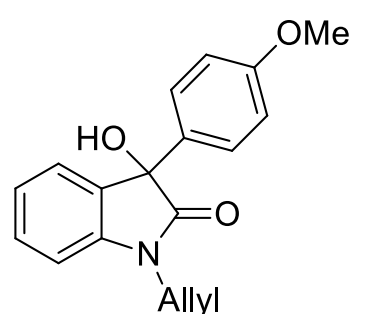
compound 1c (200 mg, $1.05 \mathrm{mmol})$, p-methoxyphenylmagnesium bromide $\mathbf{2 b}(4.2 \mathrm{~mL}, 4.20 \mathrm{mmol})$ and sodium hydride $(48.3 \mathrm{mg}, 2.10$ mmol) provided compound 7c $(217 \mathrm{mg}, 70 \%)$ as a pale yellow solid; ${ }^{1} \mathbf{H}-$ NMR (400 MHz, $\left.\mathrm{CDCl}_{3}\right): 7.31-7.25(4 \mathrm{H}, \mathrm{m}), 7.05(1 \mathrm{H}, \mathrm{t}, J=7.4 \mathrm{~Hz})$, 6.87-6.80 (3H, m), 5.87-5.77 (1H, m), 5.26-5.19 (2H, m), 4.43-4.37 (1H, 
m), $4.20(1 \mathrm{H}, \mathrm{dd}, J=10.8,5.4 \mathrm{~Hz}), 4.03(1 \mathrm{H}, \mathrm{sbr}), 3.75(3 \mathrm{H}, \mathrm{s}) ;{ }^{13} \mathbf{C}-\mathbf{N M R}\left(100 \mathrm{MHz} ; \mathrm{CDCl}_{3}\right)$ : 177.6, 159.6, 142.6, 132.4, 131.9, 131.2, 129.7, 126.9, 125.0, 123.5, 117.9, 114.1, 109.6, 77.7, 55.3, 42.6; HRMS (ESI) calcd for $\mathrm{C}_{18} \mathrm{H}_{18} \mathrm{NO}_{3}[\mathrm{M}+\mathrm{H}]^{+}:$: 296.1287; Found: 296.1279.

3-Allyl-5-bromo-3-hydroxy-1-methylindolin-2-one (7d): Using the general procedure GP-5, compound $1 \mathbf{k}$ (200 mg, $0.83 \mathrm{mmol})$, allylmagnesium bromide $2 \mathbf{c}(2.5 \mathrm{~mL}, 2.49 \mathrm{mmol})$ and

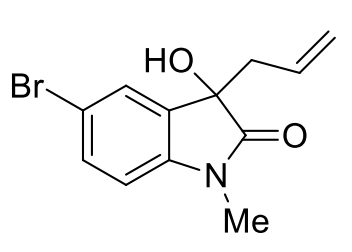
sodium hydride (40 mg, $1.66 \mathrm{mmol})$ provided compound 7d (178 mg, $76 \%)$ as a brown solid; ${ }^{1} \mathbf{H}-\mathbf{N M R}\left(500 \mathrm{MHz}, \mathrm{CDCl}_{3}\right): 7.49(1 \mathrm{H}, \mathrm{d}, J=$ $1.7 \mathrm{~Hz}), 7.45(1 \mathrm{H}, \mathrm{dd}, J=5.9,1.7 \mathrm{~Hz}), 6.70(1 \mathrm{H}, \mathrm{d}, J=8.3 \mathrm{~Hz}), 5.65-$ $5.57(1 \mathrm{H}, \mathrm{m}), 5.13(1 \mathrm{H}, \mathrm{d}, J=5.0 \mathrm{~Hz}), 5.10(1 \mathrm{H}, \mathrm{s}), 3.15(3 \mathrm{H}, \mathrm{s}), 2.71$

$(1 \mathrm{H}, \mathrm{dd}, J=7.5,5.9 \mathrm{~Hz}), 2.59(1 \mathrm{H}, \mathrm{dd}, J=8.4,5.1 \mathrm{~Hz}) ;{ }^{13} \mathbf{C}-\mathbf{N M R}\left(100 \mathrm{MHz} ; \mathrm{CDCl}_{3}\right): 177.5$, 142.5, 132.6, 131.9, 130.1, 127.6, 121.0, 115.9, 110.0, 76.0, 43.0, 26.4; HRMS (ESI) calcd for $\mathrm{C}_{12} \mathrm{H}_{12} \mathrm{BrNNaO}_{2}[\mathrm{M}+\mathrm{Na}]^{+}:$303.9949; Found: 303.9947.

3-Allyl-1-benzyl-3-hydroxy-5-(trifluoromethoxy)indolin-2-one (7e): Using the general procedure GP-5, compound 1s (200 mg, $0.62 \mathrm{mmol}$ ), allylmagnesium bromide $2 \mathbf{c}(2.0 \mathrm{~mL}, 1.86$<smiles>C=CCC1(O)C(=O)Nc2ccc(OC(F)(F)F)cc21</smiles>
$\mathrm{mmol}$ ) and sodium hydride (30 $\mathrm{mg}, 1.24 \mathrm{mmol}$ ) provided compound $7 \mathrm{e}$ (162 mg, $72 \%$ ) as a brown solid; ${ }^{1} \mathbf{H}-\mathbf{N M R}\left(500 \mathrm{MHz}, \mathrm{CDCl}_{3}\right)$ : $7.32-$ $7.23(6 \mathrm{H}, \mathrm{m}), 7.04(1 \mathrm{H}, \mathrm{dd}, J=6.7,1.7 \mathrm{~Hz}), 6.65(1 \mathrm{H}, \mathrm{d}, J=8.4 \mathrm{~Hz})$, 5.59-5.54 (1H, m), $5.07(2 \mathrm{H}, \mathrm{t}, J=8.4 \mathrm{~Hz}), 5.00(1 \mathrm{H}, \mathrm{d}, J=15.9 \mathrm{~Hz})$, $4.84(1 \mathrm{H}, \mathrm{s}), 4.66(1 \mathrm{H}, \mathrm{d}, J=15.9 \mathrm{~Hz}), 2.84(1 \mathrm{H}, \mathrm{dd}, J=6.8,6.7 \mathrm{~Hz}), 2.71(1 \mathrm{H}, \mathrm{dd}, J=8.4,4.2$ $\mathrm{Hz}) ;{ }^{13} \mathrm{C}-\mathrm{NMR}\left(100 \mathrm{MHz} ; \mathrm{CDCl}_{3}\right): 178.5,145.2,140.9,135.0,131.7,130.1,128.9,127.9$, $127.4,124.4,122.5,121.9,120.9,119.3,118.4,116.8\left(\mathrm{q}, J_{\mathrm{C}-\mathrm{F}}=255.2 \mathrm{~Hz}\right), 110.1,76.4,44.1$, 42.9; HRMS (ESI) calcd for $\mathrm{C}_{19} \mathrm{H}_{17} \mathrm{~F}_{3} \mathrm{NO}_{3}[\mathrm{M}+\mathrm{H}]^{+}: 364.1161$; Found: 364.1172.

9.0 General procedure for the synthesis of 3-aryl or allyl-3-methoxy oxindoles 8a-b from 2hydroxyindoxyls 3 (GP-6):

To a solution of 2-aryl or allyl-2-hydroxyindoxyls 3 (1 equiv.) in dry DMF (5 mL) was added sodium hydride ( 2 equiv.) portionwise at $0{ }^{\circ} \mathrm{C}$ and the resulting mixture was stirred at room temperature for $10 \mathrm{~min}$. Then methyl iodide (1.5 equiv.) was added to the reaction mixture and it 
was stirred at room temperature for $6 \mathrm{~h}$. After completion of reaction as monitored by the TLC, the reaction mixture was quenched with water followed by extraction with EtOAc $(3 \times 5 \mathrm{~mL})$. The combined organic phases were washed with brine, dried over anhydrous $\mathrm{Na}_{2} \mathrm{SO}_{4}$, filtered and concentrated in vacuo. The crude residue was then purified by column chromatography on silica gel with ethylacetate-hexane (5/95 to $15 / 85)$ to give compounds 8a-b (Scheme S9).

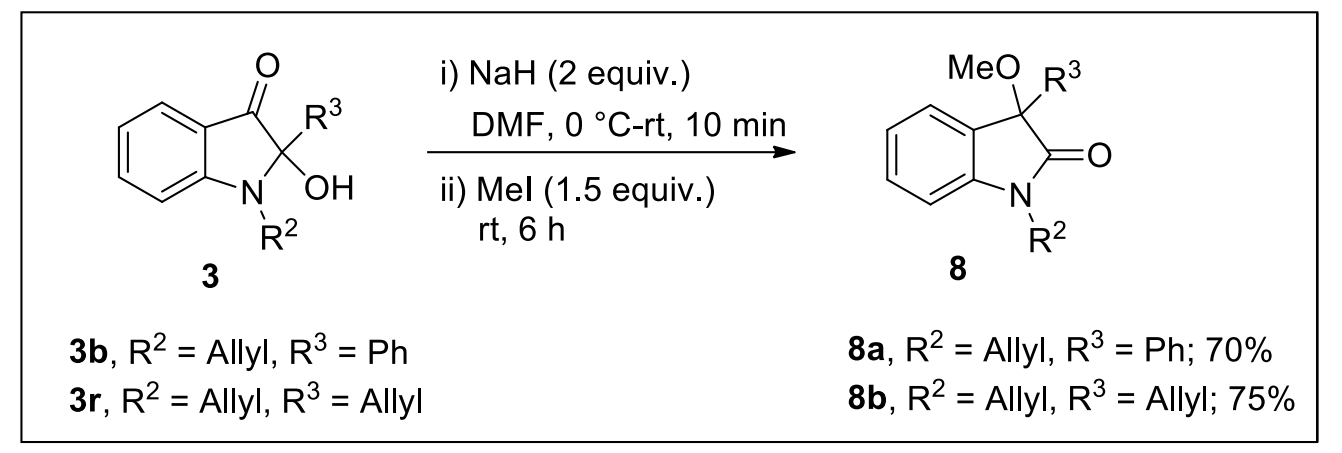

Scheme S9. Synthesis of 3-aryl or allyl-3-methoxy oxindoles 8 from 2-hydroxyindoxyls 3.

1-Allyl-3-methoxy-3-phenylindolin-2-one (8a): Using the general procedure GP-6, compound 3b (100 mg, $0.38 \mathrm{mmol})$, sodium hydride $(18 \mathrm{mg}, 0.76 \mathrm{mmol})$ and methyl iodide $(0.8 \mathrm{~mL}, 0.57$ mmol) provided compound 8a (74 $\mathrm{mg}, 70 \%)$ as a pale yellow solid; ${ }^{1} \mathbf{H}-\mathbf{N M R}$ (400 MHz,<smiles>CCCCCCN1C(=O)C(OC)(c2ccccc2)c2ccccc21</smiles>
$\left.\mathrm{CDCl}_{3}\right): 7.40-7.26(7 \mathrm{H}, \mathrm{m}), 7.13(1 \mathrm{H}, \mathrm{t}, J=7.8 \mathrm{~Hz}), 6.91(1 \mathrm{H}, \mathrm{d}, J=7.8 \mathrm{~Hz})$, 5.89-5.79 (1H, m), 5.26-5.21 (2H, m), 4.42-4.31 (2H, m), $3.25(3 \mathrm{H}, \mathrm{s}) ;{ }^{13} \mathrm{C}-$ NMR (100 MHz; $\left.\mathrm{CDCl}_{3}\right): 175.1,143.9,138.9,131.4,130.2,128.6,128.5$, 128.2, 126.4, 126.0, 123.4, 118.0, 109.7, 84.1, 53.3, 42.6; HRMS (ESI) calcd for $\mathrm{C}_{18} \mathrm{H}_{17} \mathrm{NNaO}_{2}[\mathrm{M}+\mathrm{Na}]^{+}:$302.1157; Found: 302.1156 .

1,3-Diallyl-3-methoxyindolin-2-one (8b): Using the general procedure GP-6, compound 3r (100 $\mathrm{mg}, 0.44 \mathrm{mmol})$, sodium hydride $(21 \mathrm{mg}, 0.88 \mathrm{mmol})$ provided compound $\mathbf{8 b}(80 \mathrm{mg}, 75 \%)$ as a

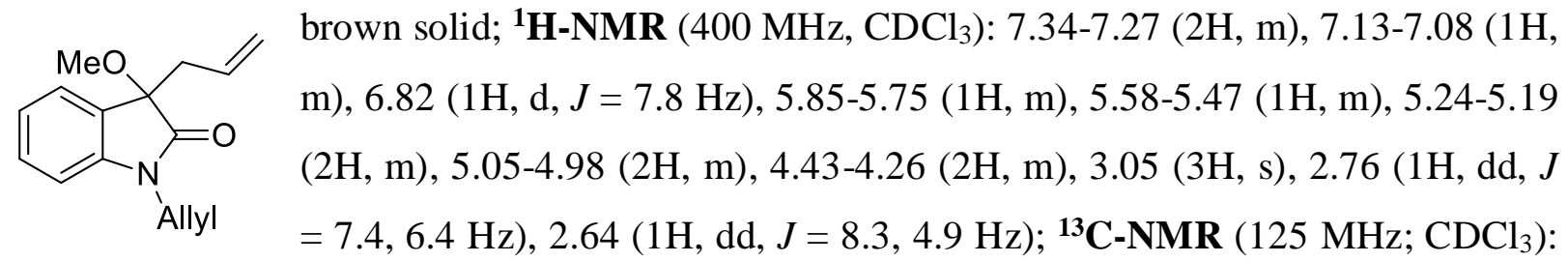


175.5, 143.3, 131.4, 130.8, 129.8, 128.3, 127.8, 126.8, 124.7, 123.0, 120.0, 117.8, 109.4, 82.6, 53.1, 42.4, 42.2; HRMS (ESI) calcd for $\mathrm{C}_{15} \mathrm{H}_{18} \mathrm{NO}_{2}[\mathrm{M}+\mathrm{H}]^{+}:$244.1338; Found: 244.1342.

\subsection{Synthesis of bis-indoxyl spirofuran derivative 9: ${ }^{3}$}

10.0.1 Synthesis of bis-indoxyl spirofuran 9a-b from 3-hydroxy-2-oxindoles 1:

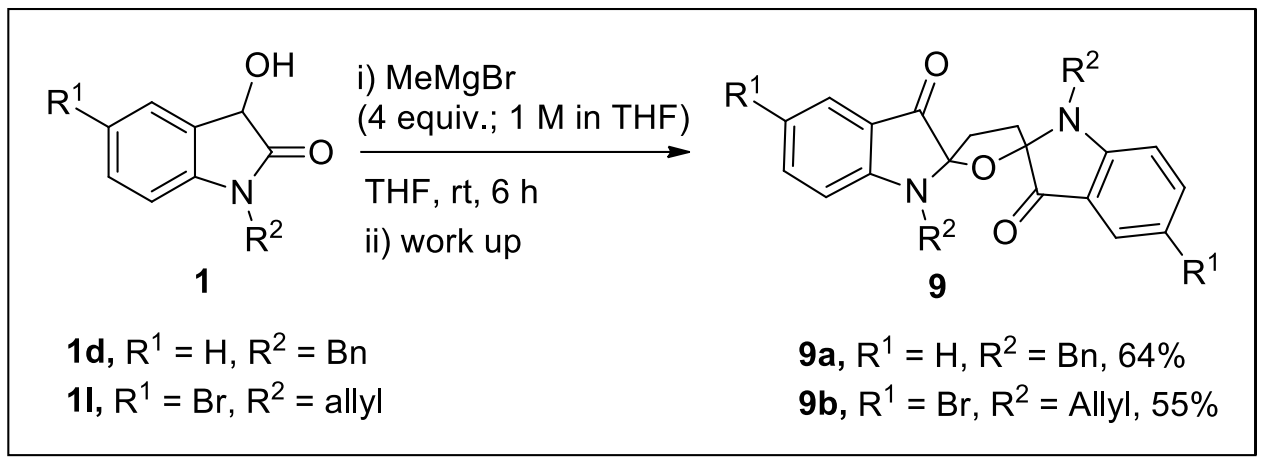

Scheme S10. Synthesis of spiroindoxyl dimer 9 from 3-hydroxyoxindoles 1.

Bis-indoxyl spirofuran (9a): Using the general procedure GP-1, compound 1c (200 mg, 0.84 $\mathrm{mmol})$ and methylmagnesium bromide $2 \mathbf{e}(3.4 \mathrm{~mL}, 3.36 \mathrm{mmol})$ provided compound $\mathbf{9 a}(262 \mathrm{mg}$,<smiles>O=C1c2ccccc2C1OCC1(N(Br)c2ccccc2)c2ccccc2N1Cc1ccccc1</smiles>
$64 \%)$ as a deep green liquid; ${ }^{1} \mathbf{H}-\mathbf{N M R}\left(500 \mathrm{MHz}, \mathrm{CDCl}_{3}\right): 7.55(2 \mathrm{H}$, d, $J=7.6 \mathrm{~Hz}), 7.36-7.23(12 \mathrm{H}, \mathrm{m}), 6.72(2 \mathrm{H}, \mathrm{t}, J=7.6 \mathrm{~Hz}), 6.47(2 \mathrm{H}$, $\mathrm{d}, J=8.4 \mathrm{~Hz}), 5.05(2 \mathrm{H}, \mathrm{d}, J=16.8 \mathrm{~Hz}), 4.68(2 \mathrm{H}, \mathrm{d}, J=16.8 \mathrm{~Hz})$, 2.71-2.64 (2H, m), 2.18-2.11 (2H, m); ${ }^{13} \mathbf{C}-\mathbf{N M R}\left(125 \mathrm{MHz}, \mathrm{CDCl}_{3}\right)$ : 201.0, 160.7, 138.6, 138.5, 128.8, 127.1, 126.8, 125.3, 118.4, 117.6, 109.4, 97.3, 44.7, 29.6; HRMS (ESI) calcd for $\mathrm{C}_{32} \mathrm{H}_{27} \mathrm{~N}_{2} \mathrm{O}_{3}[\mathrm{M}+\mathrm{H}]^{+}:$487.2022; Found: 487.2025 .

Bis-indoxyl spirofuran (9b): Using the general procedure GP-1, compound 1i (200 mg, 1.10<smiles>CN1c2ccc(F)cc2C(=O)C12CC1(O)C(=O)c3cc(F)ccc3N12</smiles>
mmol) and methylmagnesium bromide $2 \mathrm{e}(4.4 \mathrm{~mL}, 4.40 \mathrm{mmol})$ provided compound $\mathbf{9 b}$ (225 $\mathrm{mg}, 55 \%$ ) as a deep green liquid; ${ }^{1} \mathbf{H}$ NMR $\left(400 \mathrm{MHz}, \mathrm{CDCl}_{3}\right): 7.24-7.19(4 \mathrm{H}, \mathrm{m}), 6.62(2 \mathrm{H}, \mathrm{dd}, J=4.4$, $3.9 \mathrm{~Hz}), 3.03(6 \mathrm{H}, \mathrm{s}), 2.75-2.72(2 \mathrm{H}, \mathrm{m}), 2.17-2.13(2 \mathrm{H}, \mathrm{m}) ;{ }^{13} \mathrm{C}-$

NMR $\left(100 \mathrm{MHz}, \mathrm{CDCl}_{3}\right): 200.7,158.0,157.0,126.2,125.9,117.8,110.6,109.5,109.4,97.8$, 28.5, 27.2; HRMS (ESI) calcd for $\mathrm{C}_{20} \mathrm{H}_{17} \mathrm{~F}_{2} \mathrm{~N}_{2} \mathrm{O}_{3}[\mathrm{M}+\mathrm{H}]^{+}$: 371.1207; Found: 371.1219. 


\subsubsection{Transformation of 2-hydroxyindoxyl 3a' to bis-indoxyl spirofuran 9c:}

The 2-hydroxyindoxyl derivative $\mathbf{3} \mathbf{a}^{\prime}$, obtained by methylmagnesium bromide $\mathbf{2 e}$ addition to $\mathbf{1 c}$ converted to the corresponding spiroindoxyl dimer 9c via spontaneous aerial oxidation on standing (Scheme S11).

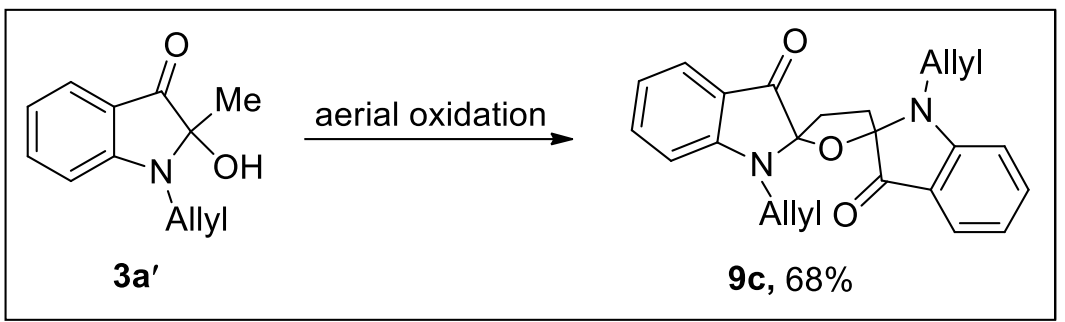

Scheme S11. Transformation of 2-hydroxyindoxyl 3a' to spiroindoxyl dimer 9c.

Bis-indoxyl spirofuran (9c): The 2-hydroxyindoxyl 3a' (155 mg, $0.76 \mathrm{mmol})$ provided compound 9c (197 mg, 68\%) as a deep green liquid; ${ }^{1} \mathbf{H}-\mathbf{N M R}\left(400 \mathrm{MHz}, \mathrm{CDCl}_{3}\right)$ : 7.53 (2H, d, $J$<smiles></smiles>
$=7.8 \mathrm{~Hz}), 7.44-7.40(2 \mathrm{H}, \mathrm{m}), 6.72(2 \mathrm{H}, \mathrm{t}, J=7.3 \mathrm{~Hz}), 6.62(2 \mathrm{H}, \mathrm{d}, J=$ $8.3 \mathrm{~Hz}), 5.95-5.85(2 \mathrm{H}, \mathrm{m}), 5.26-5.15(4 \mathrm{H}, \mathrm{m}), 4.46-4.40(2 \mathrm{H}, \mathrm{m})$, 4.05-4.00 (2H, m), 2.80-2.70 (2H, m), 2.18-2.08 (2H, m); ${ }^{13}$ C-NMR (125 MHz, $\left.\mathrm{CDCl}_{3}\right): 201.3,160.0,138.5,134.3,125.2,118.1,117.5$, 116.3, 109.3, 97.1, 43.3, 29.3; HRMS (ESI) calcd for $\mathrm{C}_{24} \mathrm{H}_{23} \mathrm{~N}_{2} \mathrm{O}_{3}[\mathrm{M}+\mathrm{H}]^{+}$: 387.1709; Found: 387.1723.

11.0 X-Ray Crystallography of compound 3g and 9c: Intensity data was collected on a Bruker's Kappa Apex II CCD Duo diffractometer with graphite monochromated $\mathrm{Mo}_{\mathrm{K \alpha}}$ radiation $(0.71073 \AA)$ at the temperature of $296 \mathrm{~K}$. Scaling and multi-scan absorption correction were employed using SADABS. The structure was solved by direct methods and all the non-hydrogen atoms were refined anisotropically while the hydrogen atoms fixed in the predetermined positions by Shelxs-97 and Shelxl-97 packages respectively. 


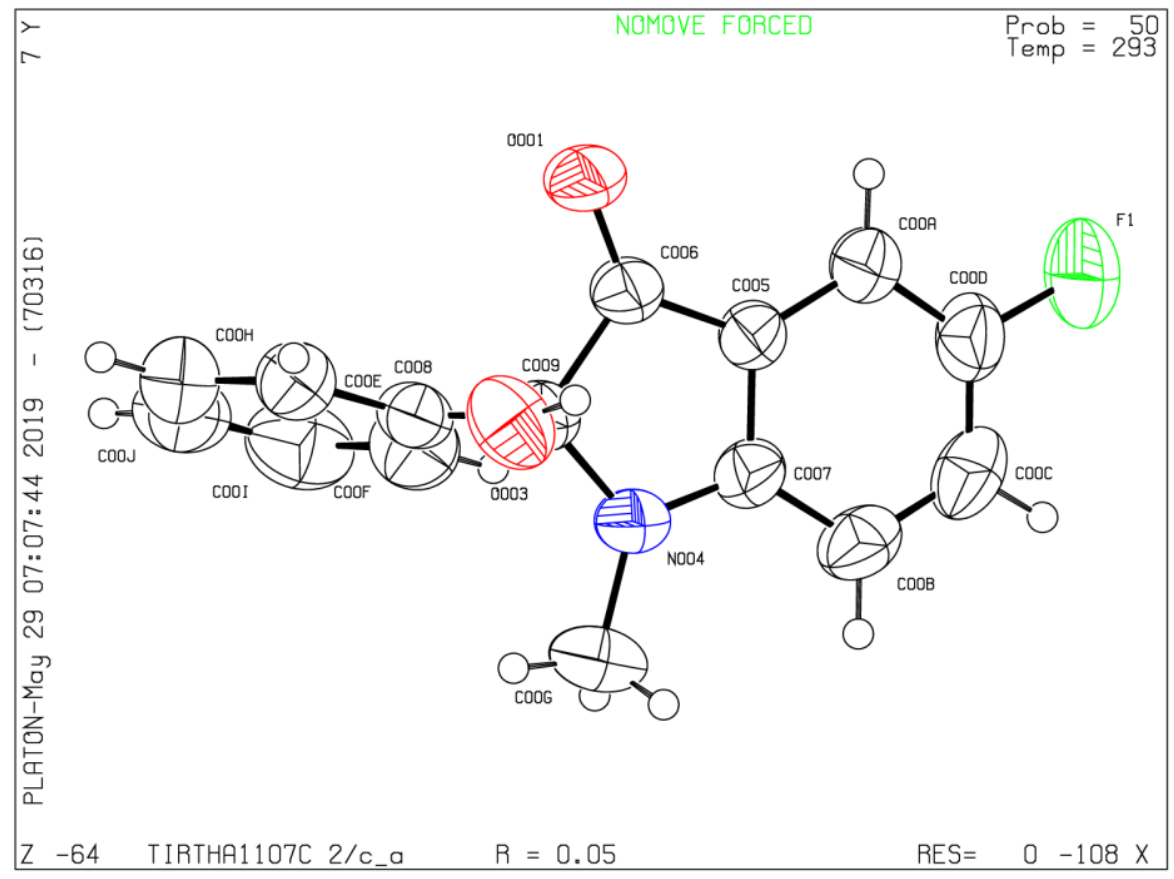

Figure S1. The ORTEP diagram of $\mathbf{3 g}$ showing $50 \%$ probability thermal ellipsoid.

\section{Crystal Data and Details of the Structure Determination for 3g:}

\begin{tabular}{|c|c|}
\hline Empirical formula & $\mathrm{C}_{15} \mathrm{H}_{12} \mathrm{FNO}_{2}$ \\
\hline Formula weight & 257.26 \\
\hline Temperature/K & 293(2) \\
\hline Crystal system & Monoclinic \\
\hline Space group & $\mathrm{C} 2 / \mathrm{c}$ \\
\hline $\mathrm{a} / \AA$ & $21.666(20)$ \\
\hline $\mathrm{b} / \AA$ & $7.148(7)$ \\
\hline $\mathrm{c} / \AA$ & $17.069(16)$ \\
\hline$\alpha /^{\circ}$ & 90 \\
\hline$\beta /{ }^{\circ}$ & $106.773(14)$ \\
\hline$\gamma /{ }^{\circ}$ & 90 \\
\hline Volume $/ \AA^{3}$ & 2531(4) \\
\hline $\mathrm{Z}$ & 8 \\
\hline$\rho_{\text {calc }} g / \mathrm{cm}^{3}$ & 1.350 \\
\hline$\mu / \mathrm{mm}^{-1}$ & 0.100 \\
\hline $\mathrm{F}(000)$ & 1072.0 \\
\hline Crystal size $/ \mathrm{mm}^{3}$ & $.14 \times .12 \times .10$ \\
\hline Radiation & $\operatorname{MoK} \alpha(\lambda=0.71073)$ \\
\hline \multicolumn{2}{|c|}{$2 \Theta$ range for data collection $/{ }^{\circ} 3.926$ to 50} \\
\hline Index ranges & $-25 \leq \mathrm{h} \leq 18,-5 \leq \mathrm{k} \leq 8,-20 \leq 1 \leq 20$ \\
\hline
\end{tabular}




\begin{tabular}{|l|l|}
\hline Reflections collected & 6095 \\
\hline Independent reflections & $2221\left[\mathrm{R}_{\text {int }}=0.0441, \mathrm{R}_{\text {sigma }}=0.0553\right]$ \\
\hline Data/restraints/parameters & $2221 / 0 / 175$ \\
\hline Goodness-of-fit on $\mathrm{F}^{2}$ & 0.942 \\
\hline Final $\mathrm{R}$ indexes [I $>=2 \sigma(\mathrm{I})]$ & $\mathrm{R}_{1}=0.0502, \mathrm{wR}_{2}=0.1361$ \\
\hline Final $\mathrm{R}$ indexes [all data] & $\mathrm{R}_{1}=0.1049, \mathrm{wR}_{2}=0.1747$ \\
\hline Largest diff. peak/hole $/ \mathrm{e} \AA^{-3}$ & $0.17 /-0.15$ \\
\hline
\end{tabular}

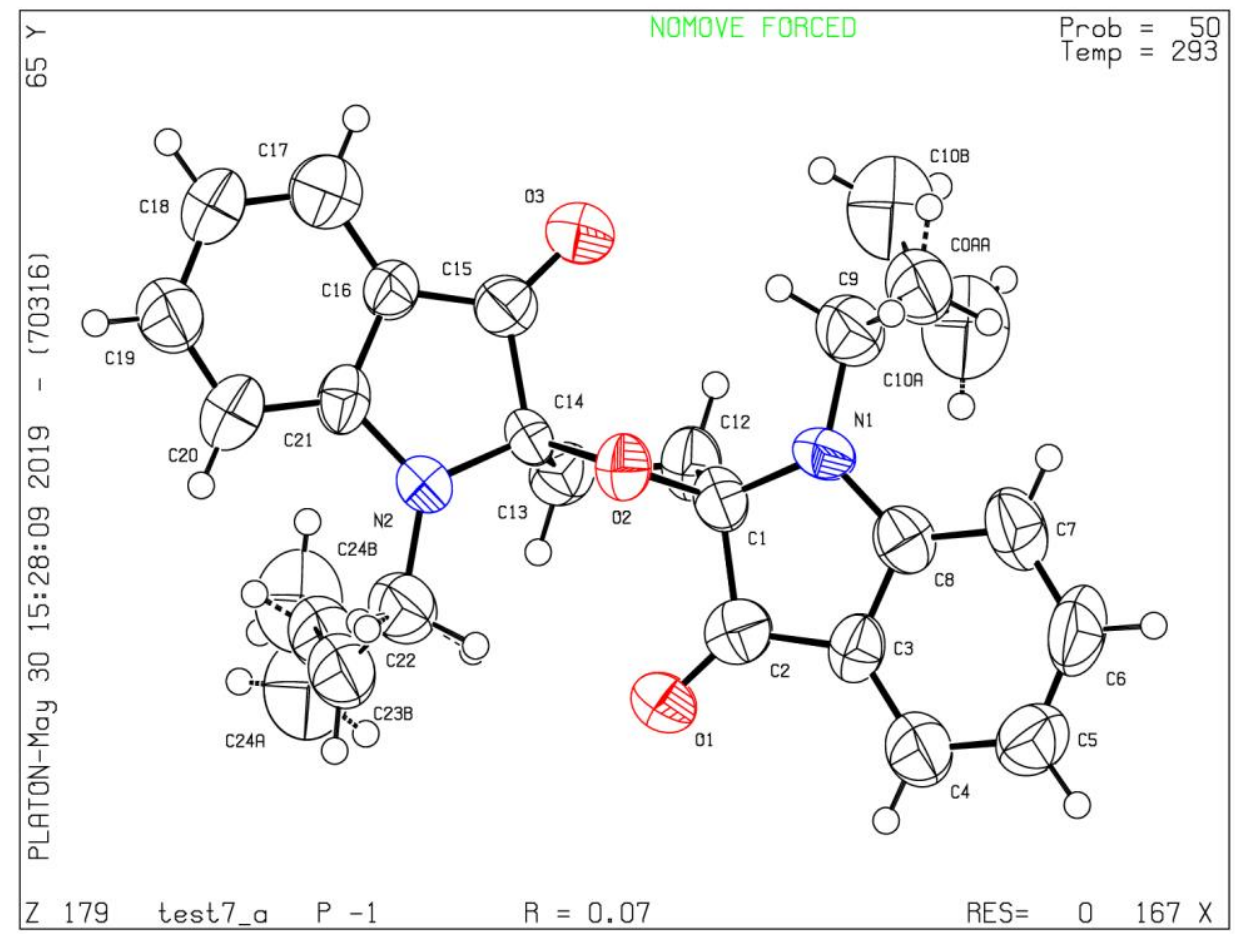

Figure S2. The ORTEP diagram of 9c showing 50\% probability thermal ellipsoid.

\section{Crystal Data and Details of the Structure Determination for 9c:}

\begin{tabular}{|l|l|}
\hline Empirical formula & $\mathrm{C}_{24} \mathrm{H}_{22} \mathrm{~N}_{2} \mathrm{O}_{3}$ \\
\hline Formula weight & 386.43 \\
\hline Temperature/K & 293.15 \\
\hline Crystal system & Triclinic \\
\hline Space group & $\mathrm{P}-1$ \\
\hline $\mathrm{a} / \AA$ & $9.468(8)$ \\
\hline $\mathrm{b} / \AA$ & $9.485(8)$ \\
\hline $\mathrm{c} / \AA$ & $11.807(11)$ \\
\hline$\alpha /{ }^{\circ}$ & $89.78(3)$ \\
\hline$\beta /{ }^{\circ}$ & $71.85(3)$ \\
\hline
\end{tabular}




\begin{tabular}{|l|l|}
\hline$\gamma /{ }^{\circ}$ & $81.09(2)$ \\
\hline Volume/ $\AA^{3}$ & $994.3(16)$ \\
\hline$Z$ & 2 \\
\hline$\rho_{\text {calcg }} / \mathrm{cm}^{3}$ & 1.291 \\
\hline$\mu / \mathrm{mm}^{-1}$ & 0.086 \\
\hline $\mathrm{F}(000)$ & 408.0 \\
\hline Crystal size/mm & $0.14 \times 0.12 \times 0.1$ \\
\hline Radiation & MoK $\alpha(\lambda=0.71073)$ \\
\hline $2 \Theta$ range for data collection/ & 3.634 to 49.992 \\
\hline Index ranges & $-11 \leq \mathrm{h} \leq 11,-11 \leq \mathrm{k} \leq 11,-11 \leq 1 \leq 14$ \\
\hline Reflections collected & 12124 \\
\hline Independent reflections & $3494\left[\mathrm{R}_{\text {int }}=0.3360, \mathrm{R}_{\text {sigma }}=0.4578\right]$ \\
\hline Data/restraints/parameters & $3494 / 1 / 254$ \\
\hline Goodness-of-fit on $\mathrm{F}^{2}$ & 0.718 \\
\hline Final R indexes [I>=2 $\sigma(\mathrm{I})]$ & $\mathrm{R}_{1}=0.0695, \mathrm{wR}_{2}=0.1092$ \\
\hline Final R indexes [all data] & $\mathrm{R}_{1}=0.3220, \mathrm{wR}_{2}=0.1717$ \\
\hline Largest diff. peak/hole $/ \mathrm{e} \AA^{-3}$ & $0.32 /-0.34$ \\
\hline
\end{tabular}

\subsection{References.}

1) Bergonzini, G.; Melchiorre, P. Angew. Chem. Int. Ed., 2012, 51, 971.

2) Kafka, S.; Klásek, A.; Košmrlj, J. J. Org. Chem. 2001, 66, 6394.

3) Aristeo-Dominguez, A.; Meléndez-Rodríguez, M.; Castillo, O. R. S.; Contreras-Martínez, Y. M. A.; Suárez-Ramírez, L.; Trejo-Carbajal, N.; Morales-Ríos, M. S.; Joseph-Nathan, P. Heterocycles, 2013, 87, 1249 
13.0 ${ }^{1} \mathrm{H}$ and ${ }^{13} \mathrm{C}$ NMR spectra of synthesized compounds:

${ }^{1} \mathrm{H}$ and ${ }^{13} \mathrm{C}$ NMR spectra of $\mathbf{3 a}$ :
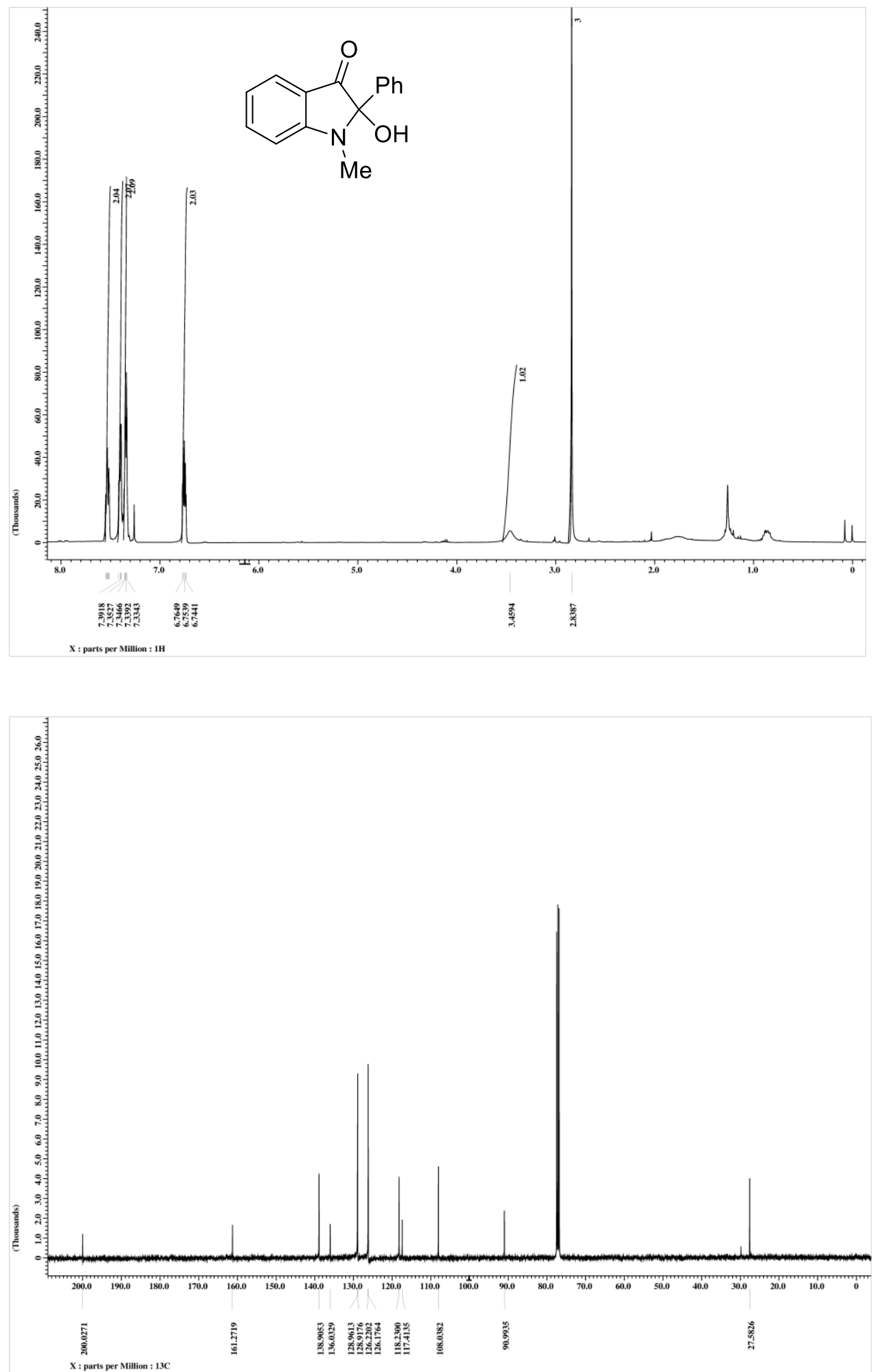
${ }^{1} \mathrm{H}$ and ${ }^{13} \mathrm{C}$ NMR spectra of $\mathbf{3 b}$ :

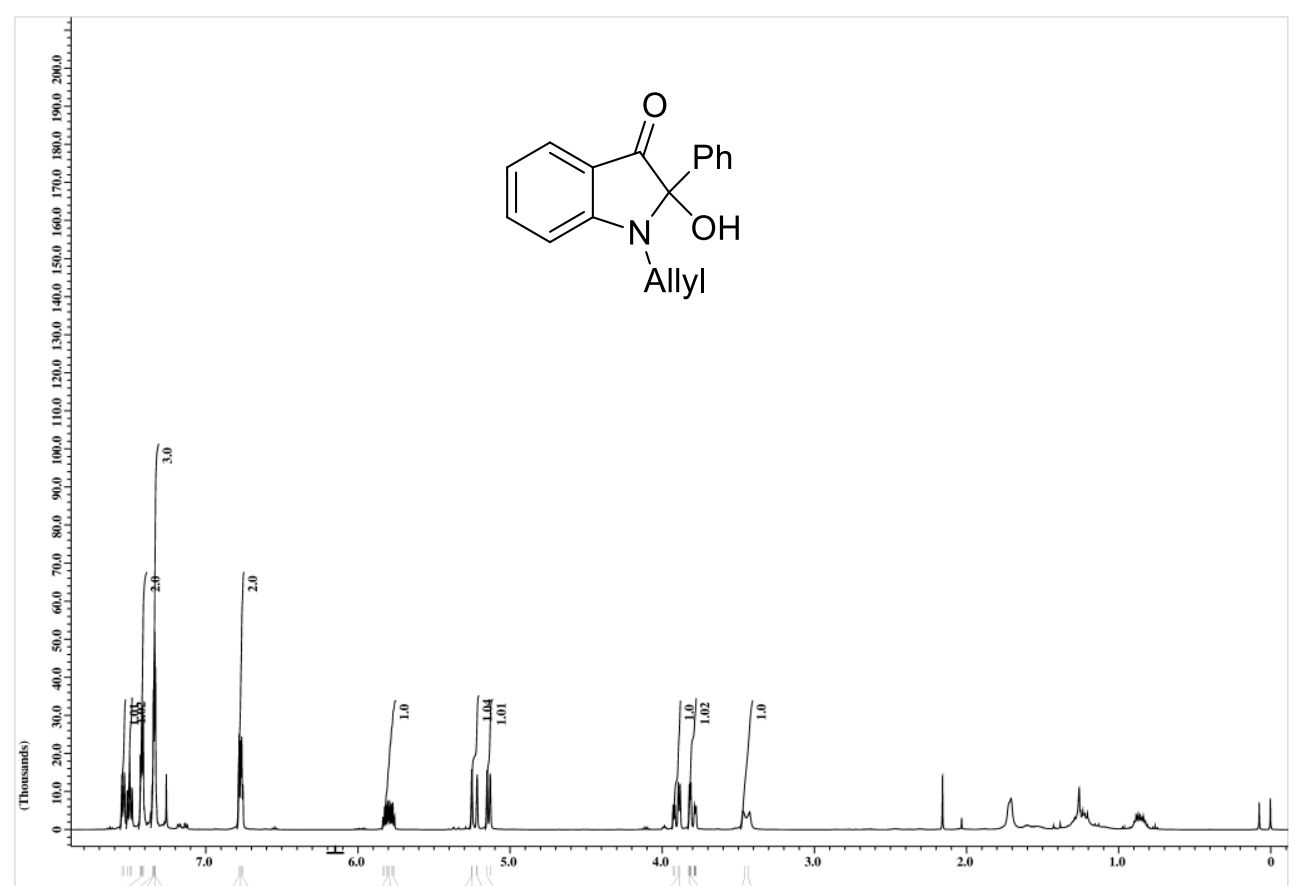

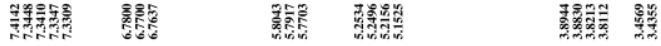

$X$ : parts per Million : $1 \mathrm{H}$

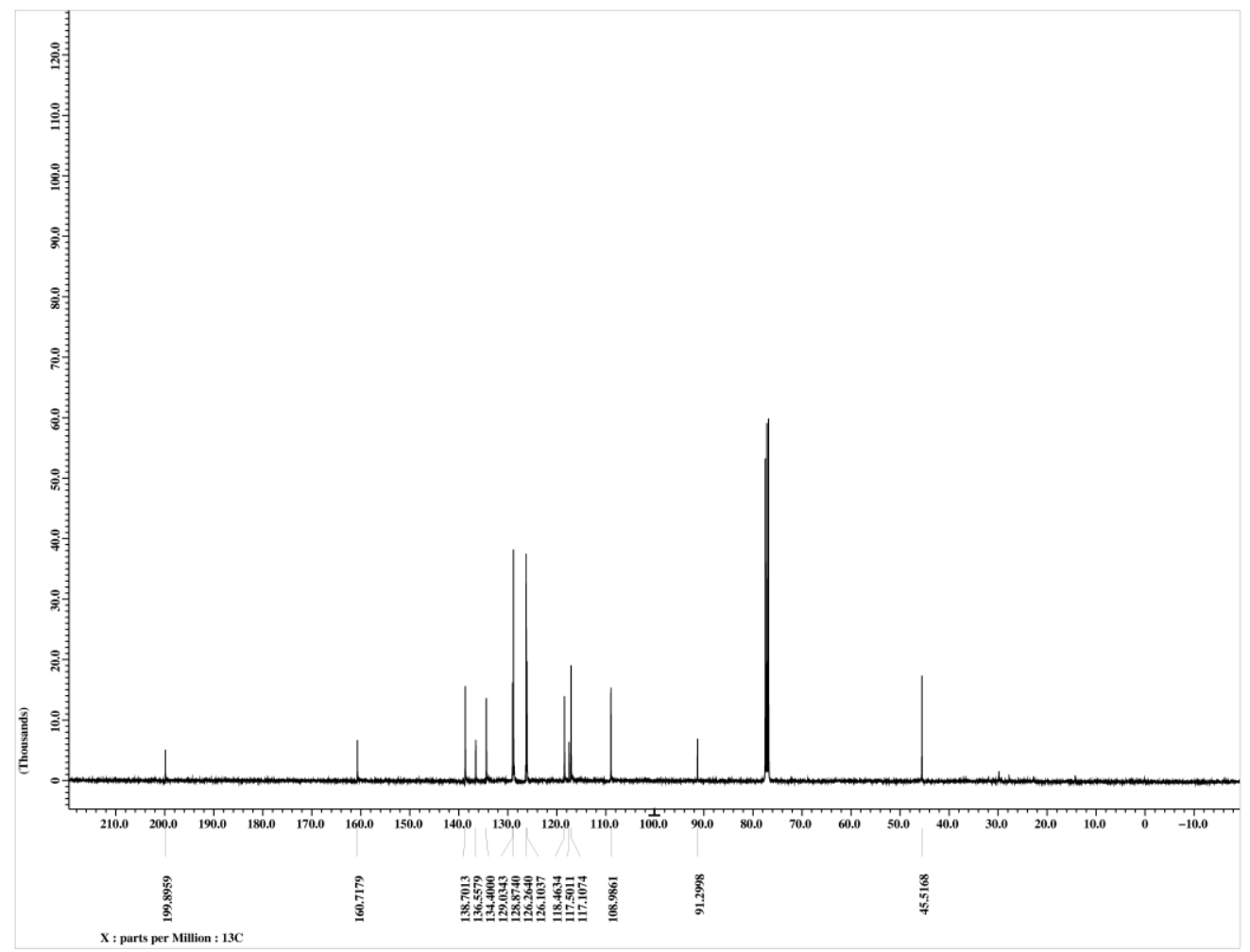


${ }^{1} \mathrm{H}$ and ${ }^{13} \mathrm{C}$ NMR spectra of $\mathbf{3 c}$ :
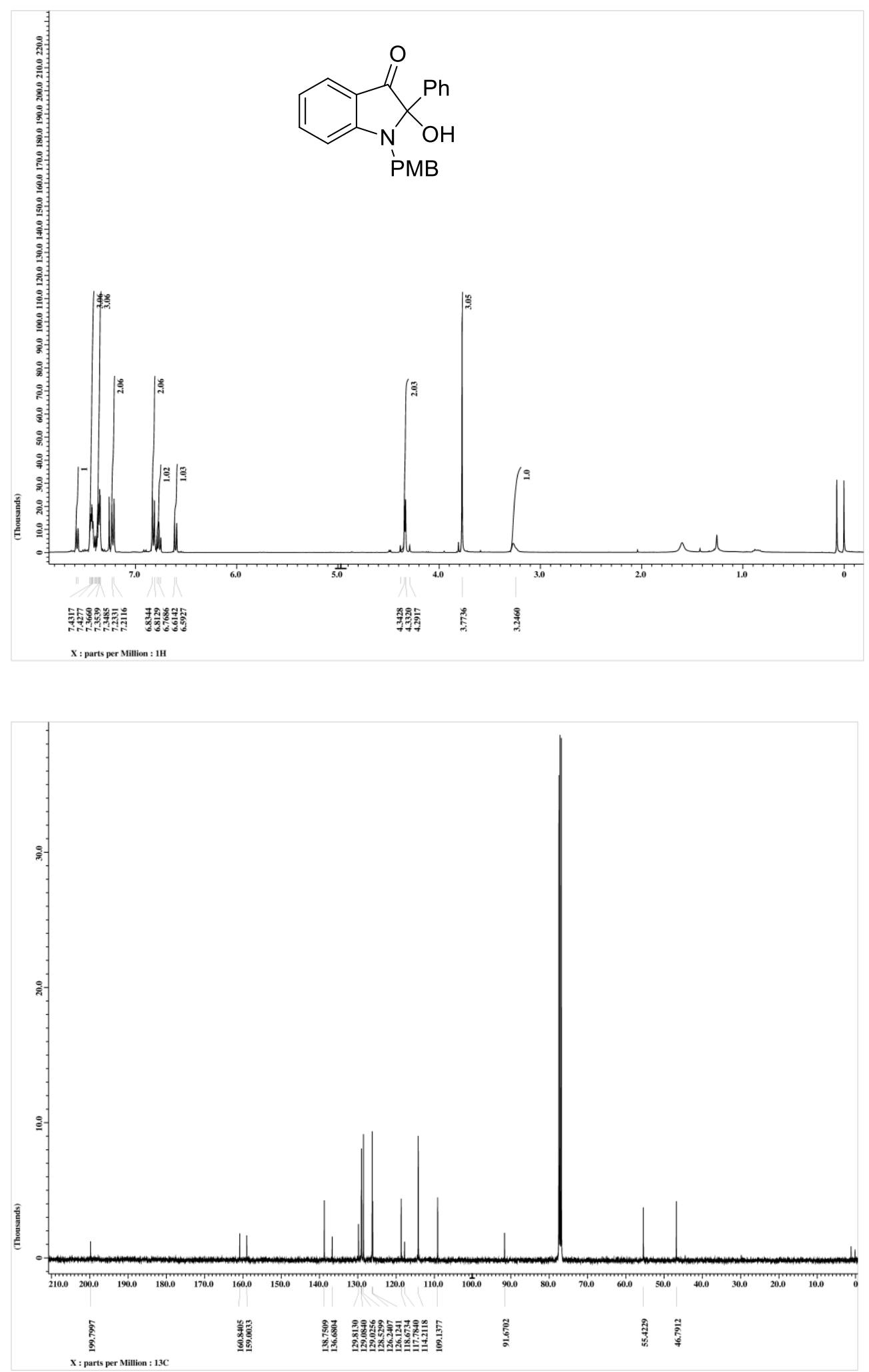
${ }^{1} \mathrm{H}$ and ${ }^{13} \mathrm{C}$ NMR spectra of $\mathbf{3 d}$ :
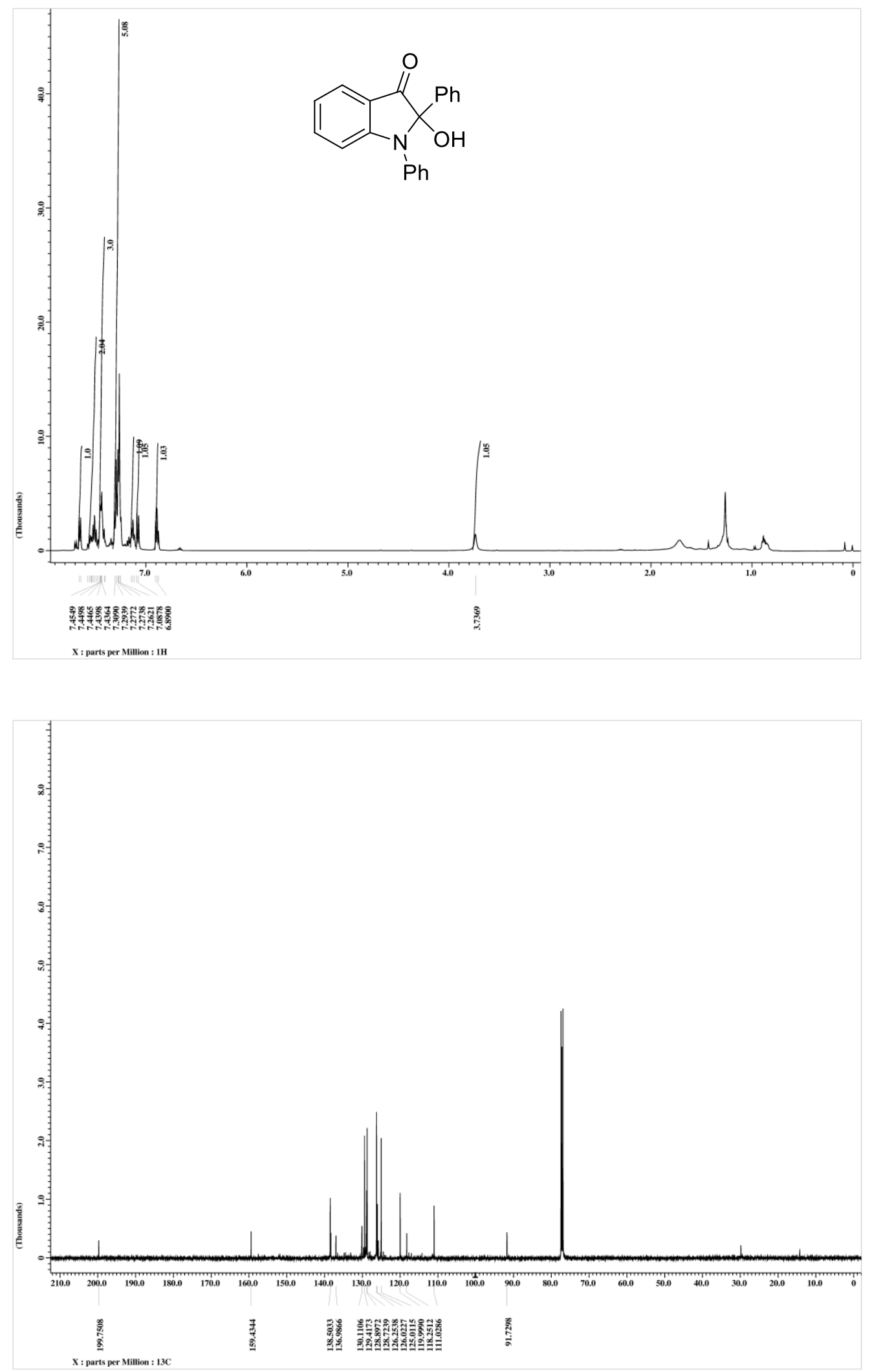
${ }^{1} \mathrm{H}$ and ${ }^{13} \mathrm{C}$ NMR spectra of $\mathbf{3 e}$ :
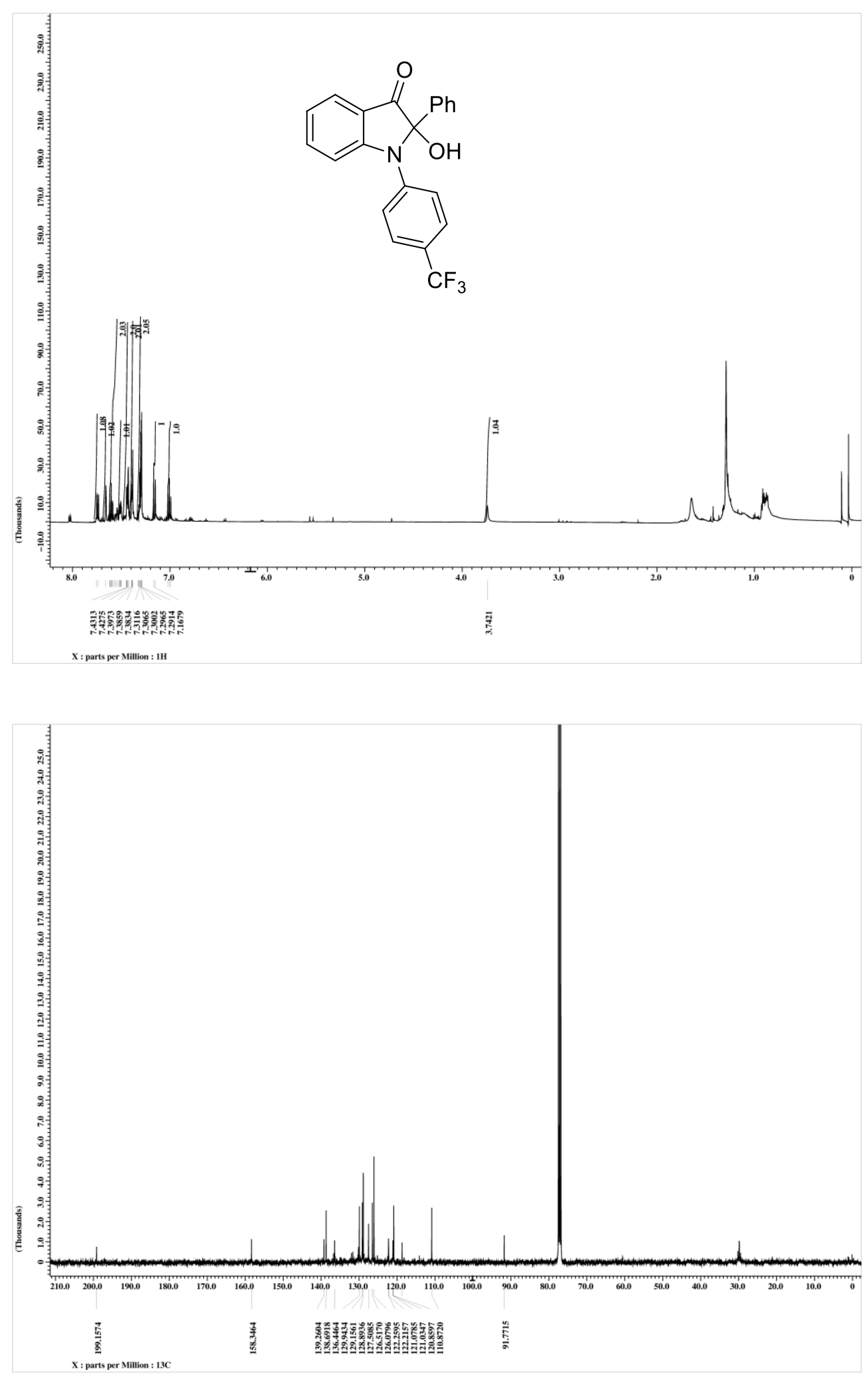
${ }^{1} \mathrm{H}$ and ${ }^{13} \mathrm{C}$ NMR spectra of $\mathbf{3 f}$ :
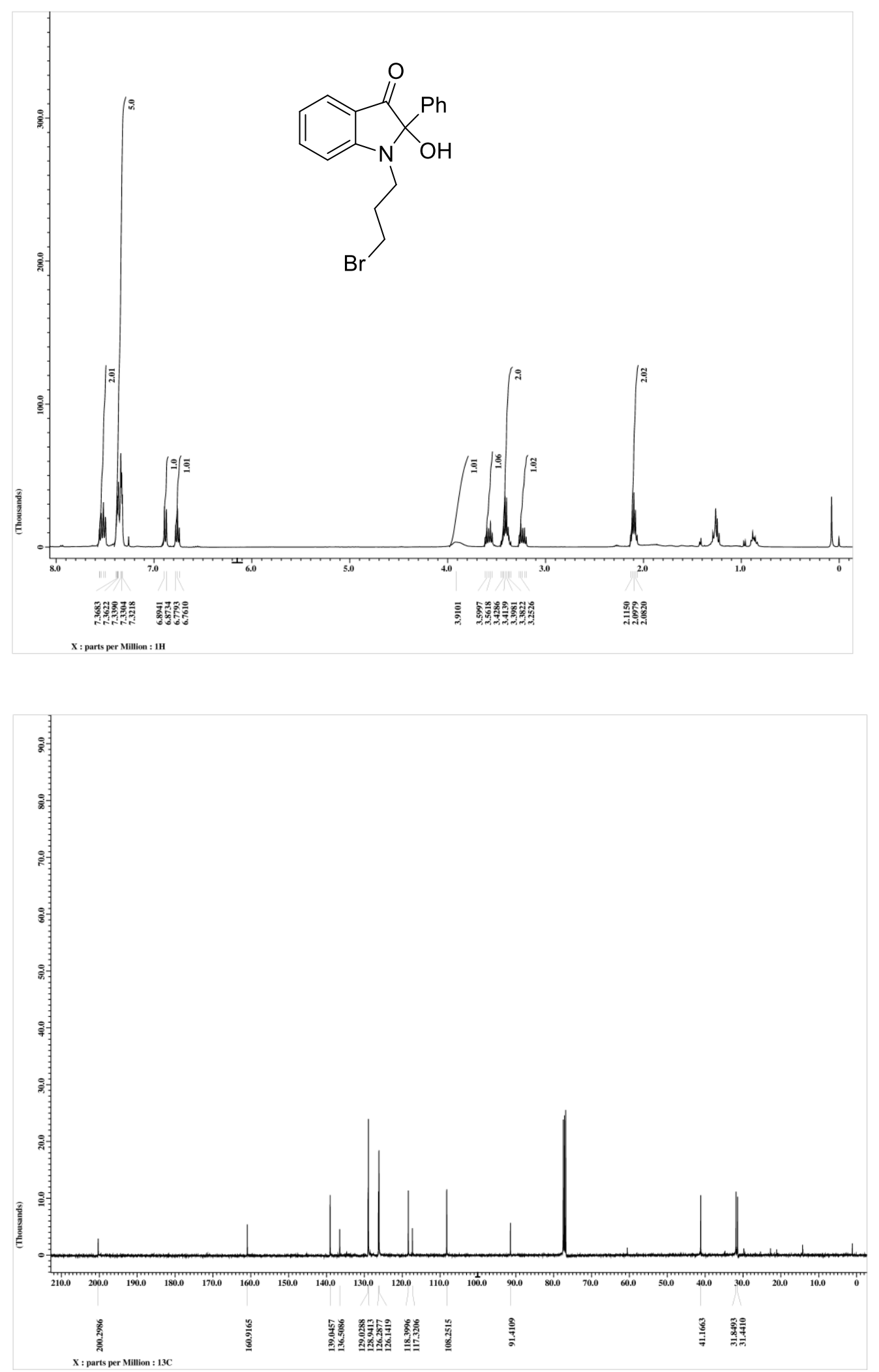
${ }^{1} \mathrm{H}$ and ${ }^{13} \mathrm{C}$ NMR spectra of $\mathbf{3 g}$ :
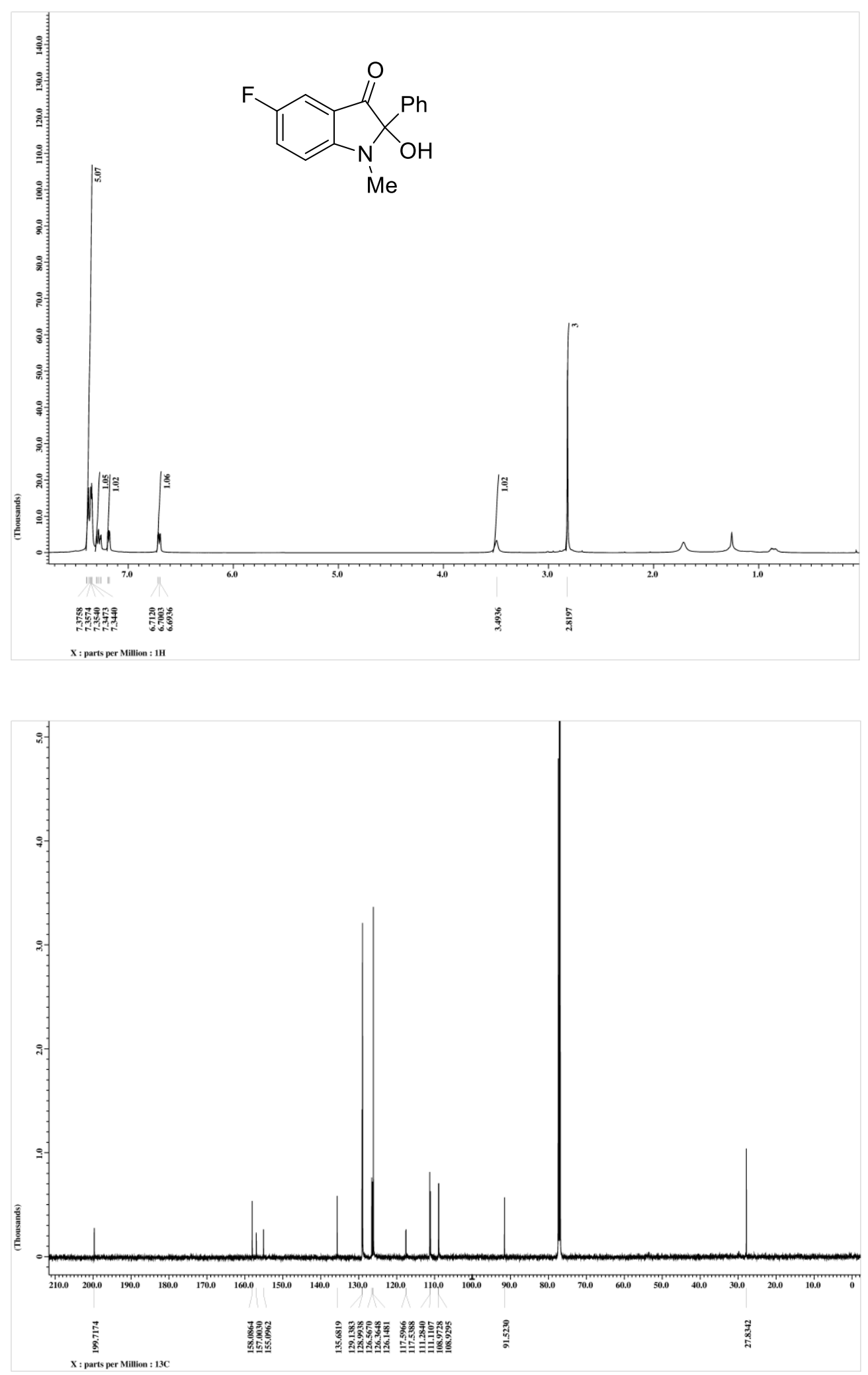
${ }^{1} \mathrm{H}$ and ${ }^{13} \mathrm{C}$ NMR spectra of $\mathbf{3 h}$ :
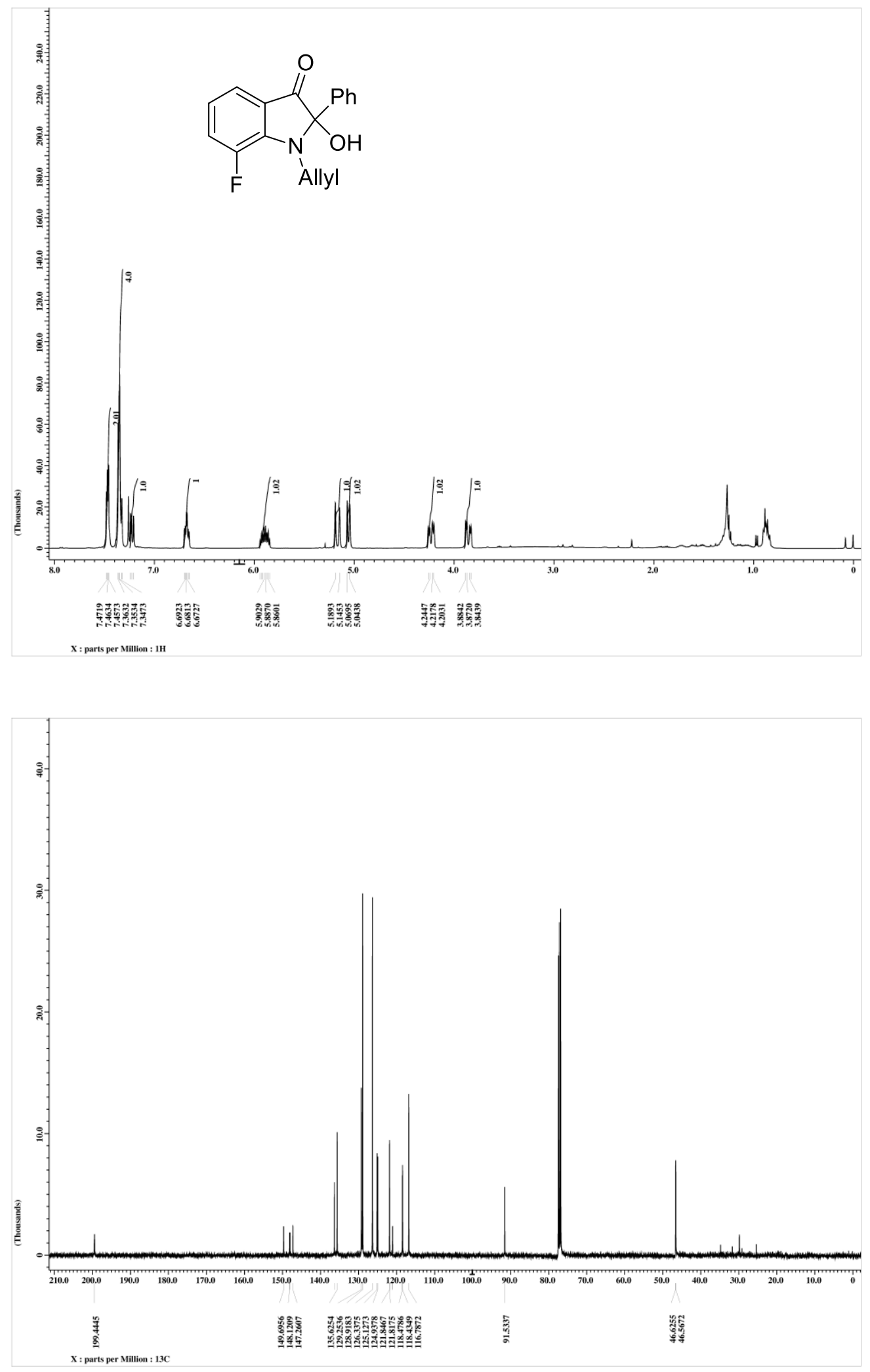
${ }^{1} \mathrm{H}$ and ${ }^{13} \mathrm{C}$ NMR spectra of $3 i$ :
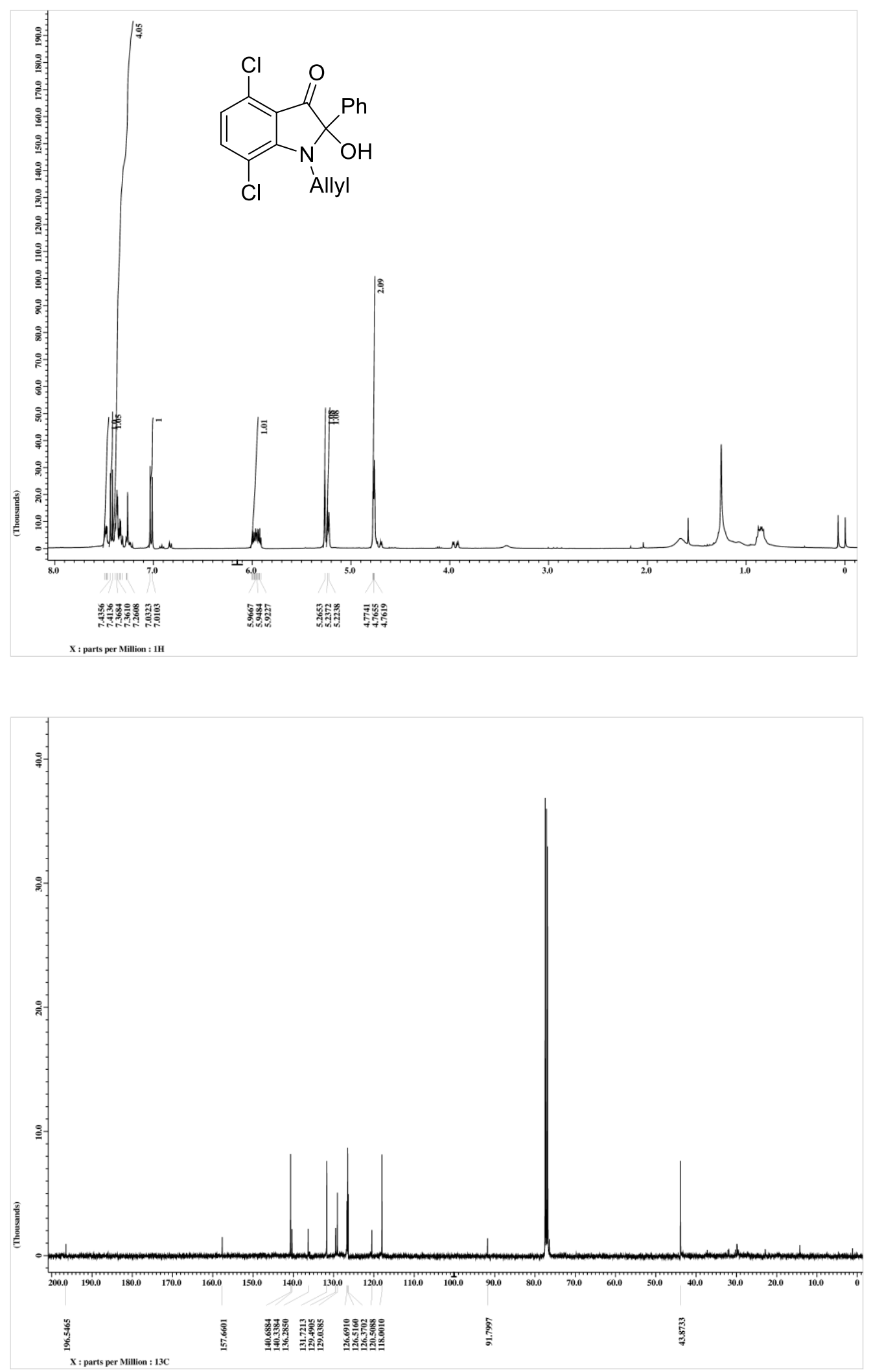
${ }^{1} \mathrm{H}$ and ${ }^{13} \mathrm{C}$ NMR spectra of $\mathbf{3 j}$ :
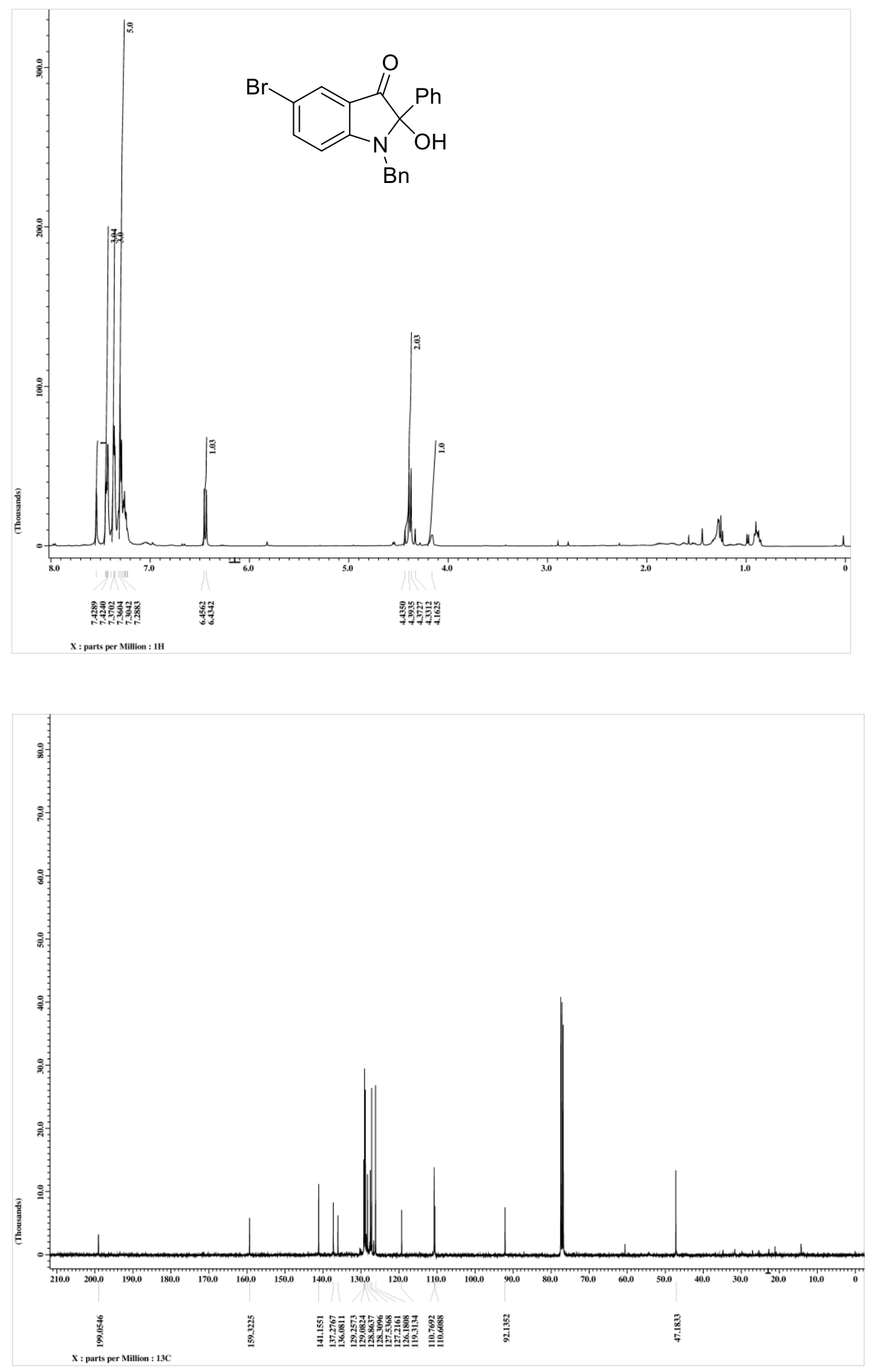
${ }^{1} \mathrm{H}$ and ${ }^{13} \mathrm{C}$ NMR spectra of $\mathbf{3 k}$ :
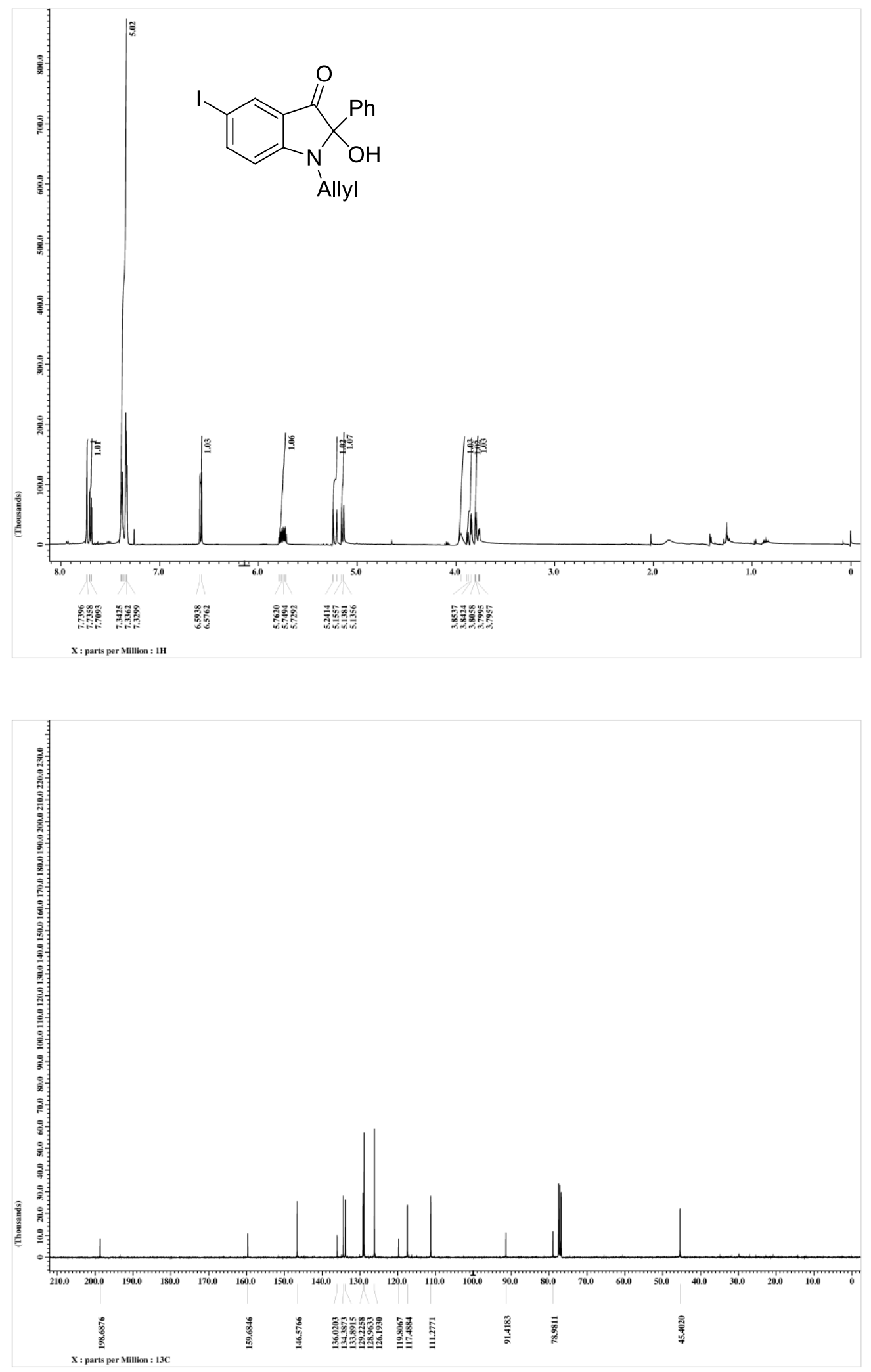
${ }^{1} \mathrm{H}$ and ${ }^{13} \mathrm{C}$ NMR spectra of $\mathbf{3 1}$ :
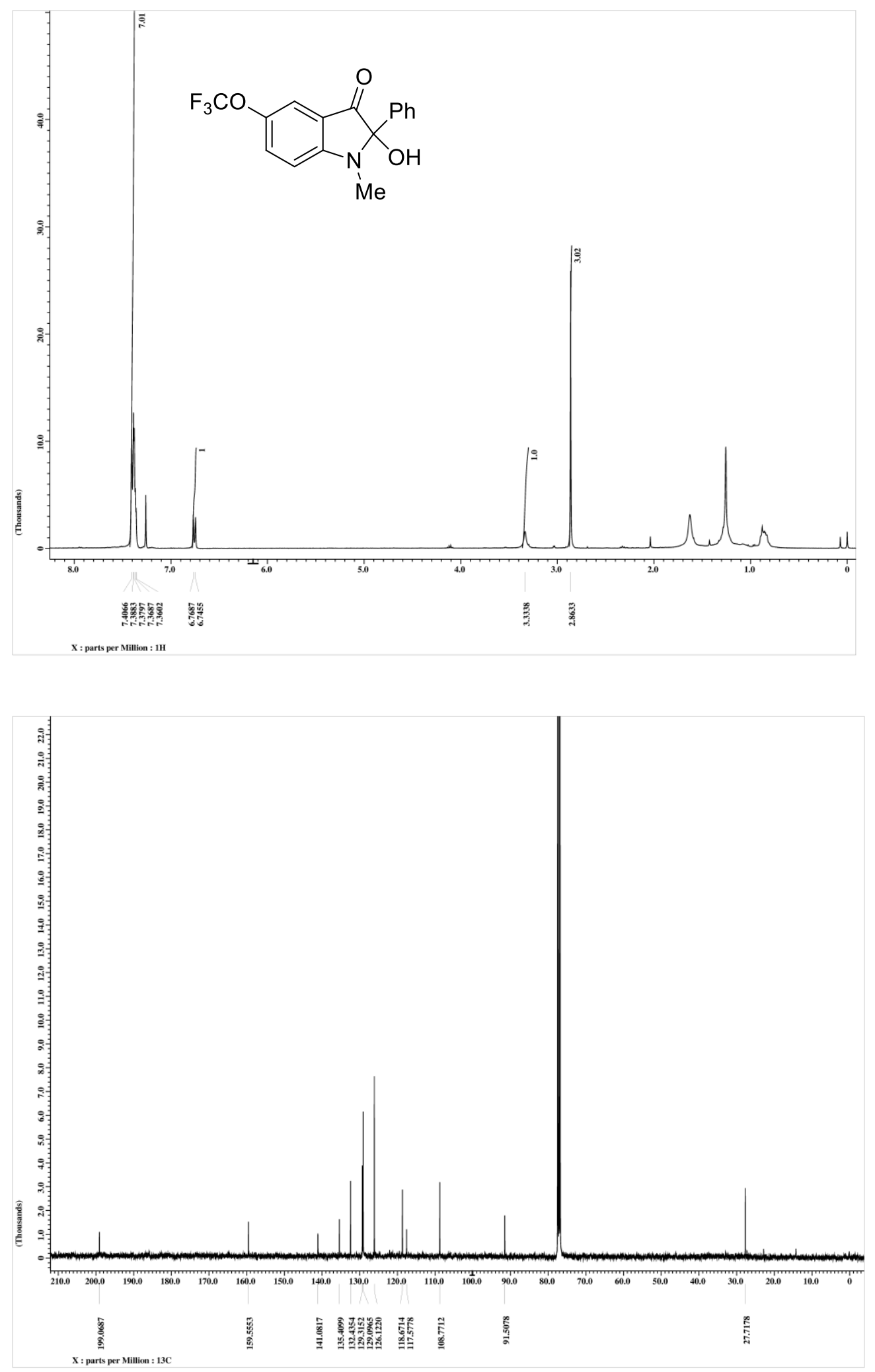
${ }^{1} \mathrm{H}$ and ${ }^{13} \mathrm{C}$ NMR spectra of $\mathbf{3 m}$ :
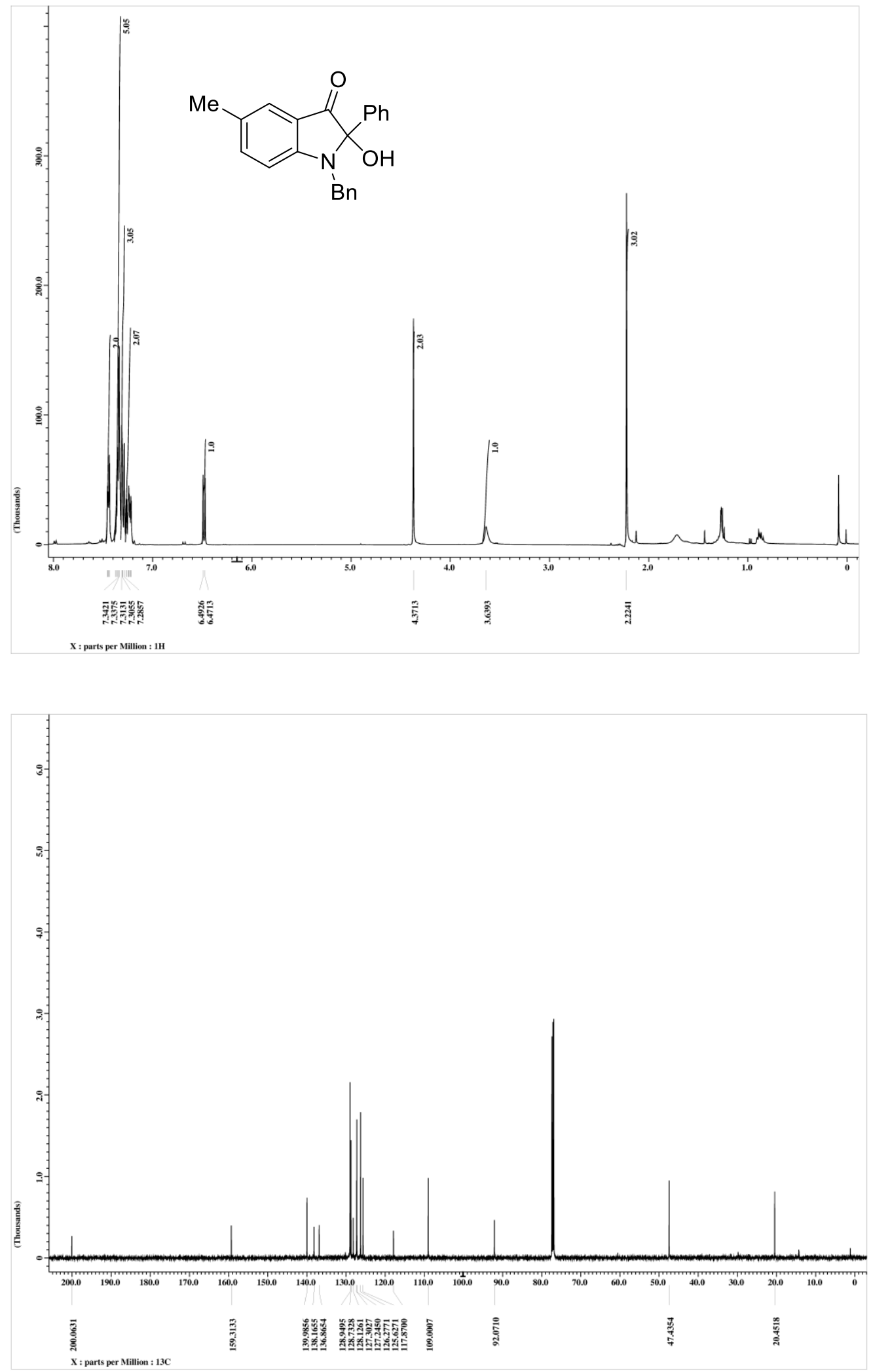
${ }^{1} \mathrm{H}$ and ${ }^{13} \mathrm{C}$ NMR spectra of $\mathbf{3 n}$ :
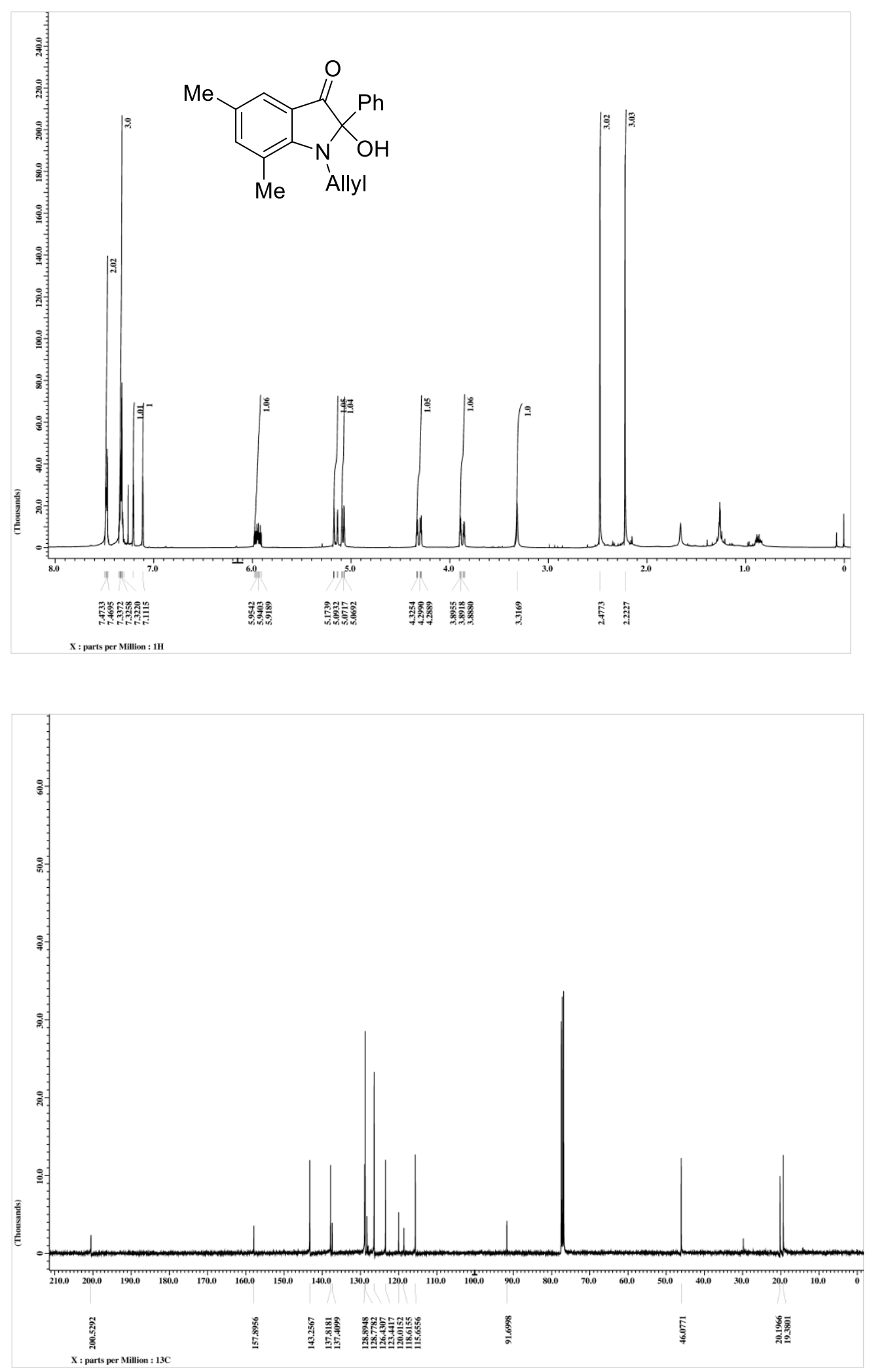
${ }^{1} \mathrm{H}$ and ${ }^{13} \mathrm{C}$ NMR spectra of $\mathbf{3 o}$ :
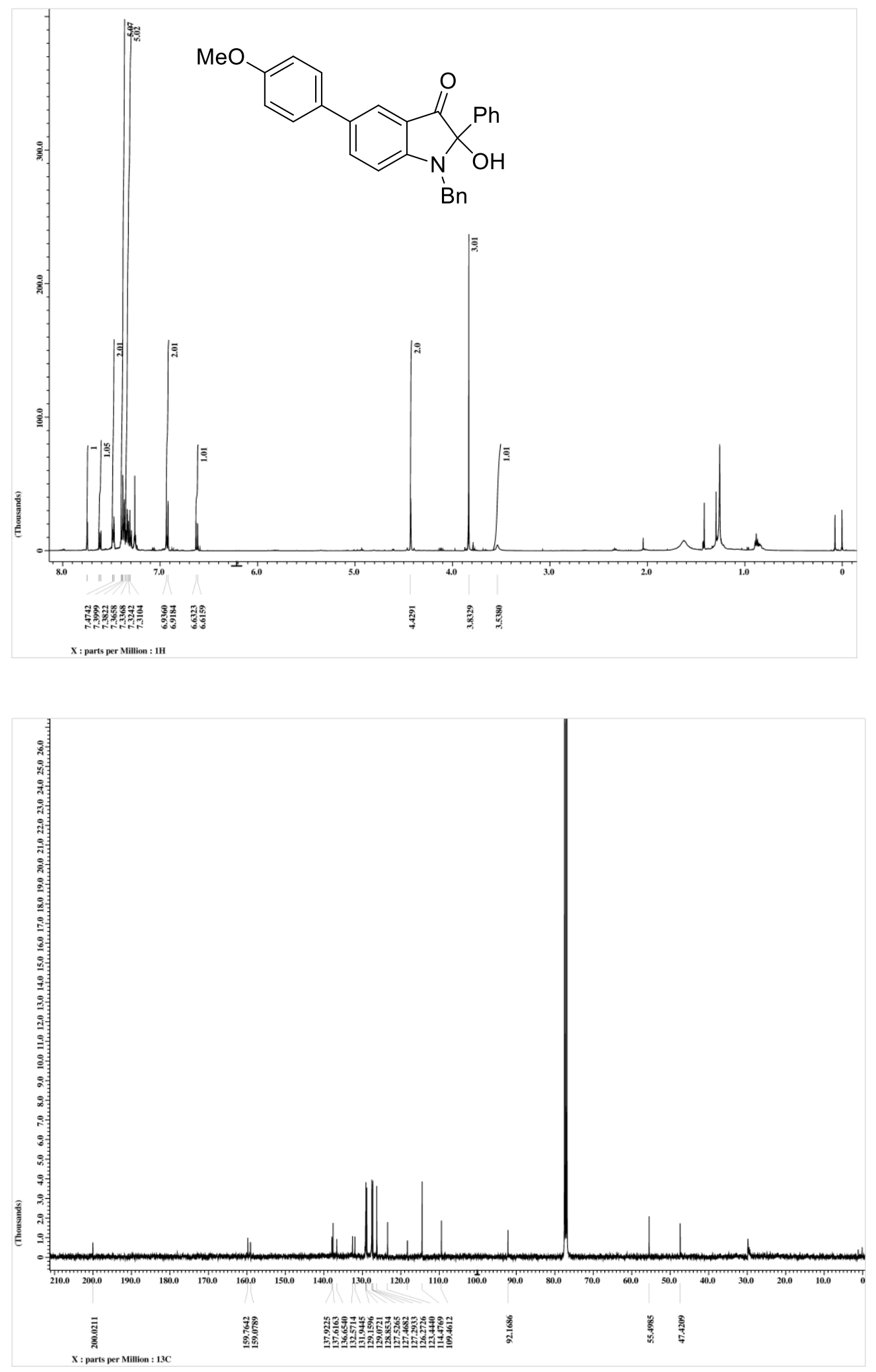
${ }^{1} \mathrm{H}$ and ${ }^{13} \mathrm{C}$ NMR spectra of $\mathbf{3 p}$ :
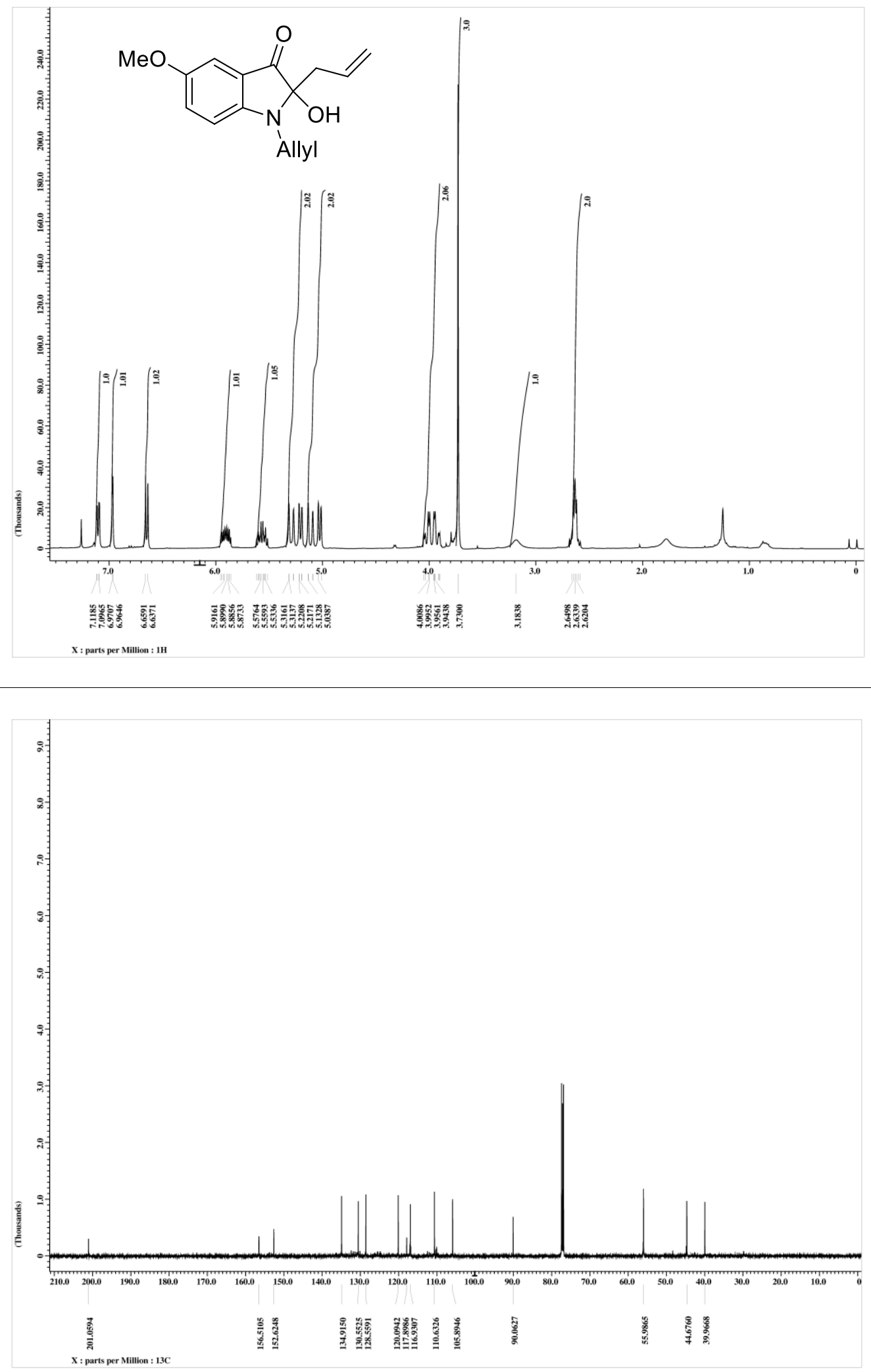
${ }^{1} \mathrm{H}$ and ${ }^{13} \mathrm{C}$ NMR spectra of 3q:
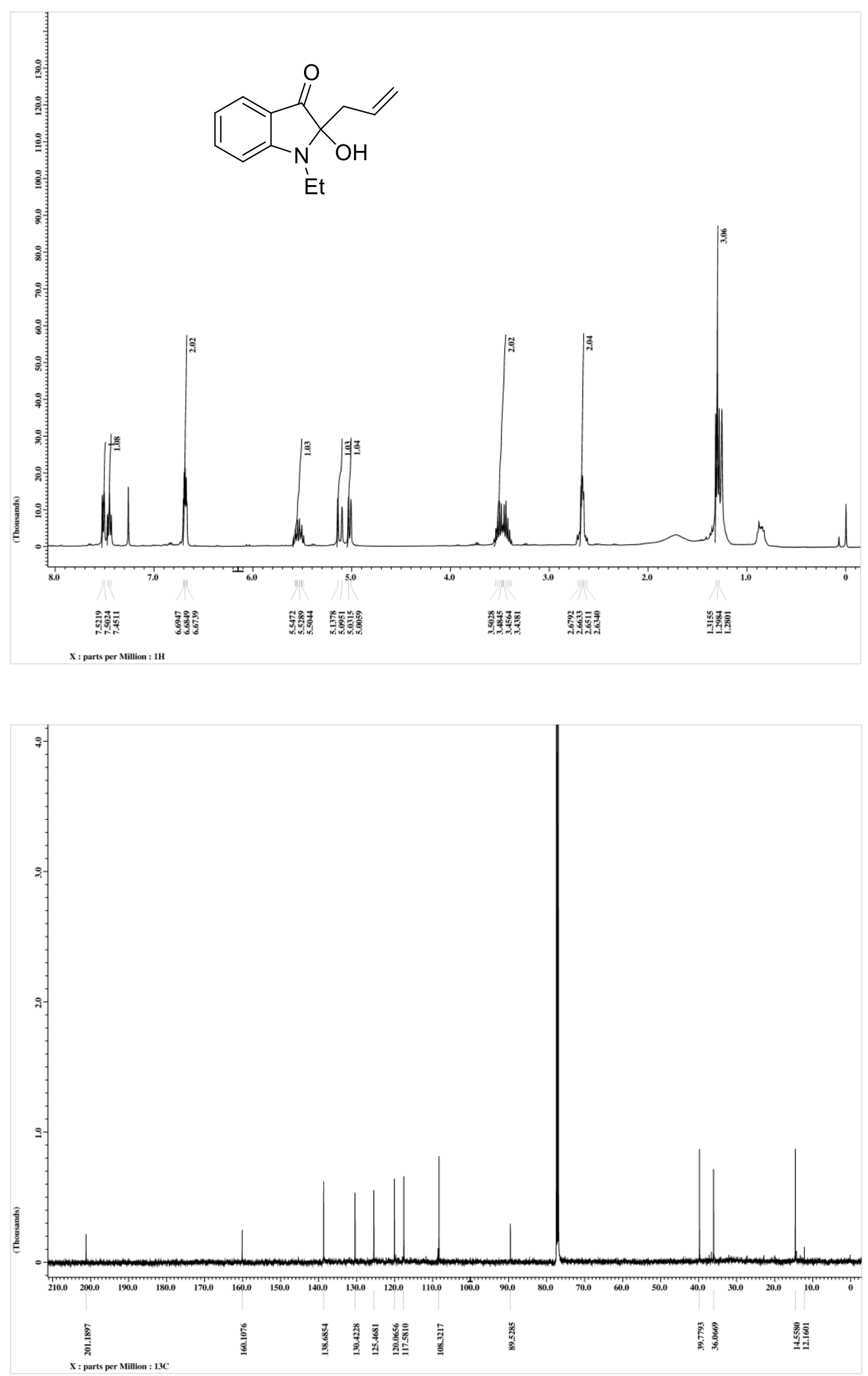
${ }^{1} \mathrm{H}$ and ${ }^{13} \mathrm{C}$ NMR spectra of $\mathbf{3 r}$ :
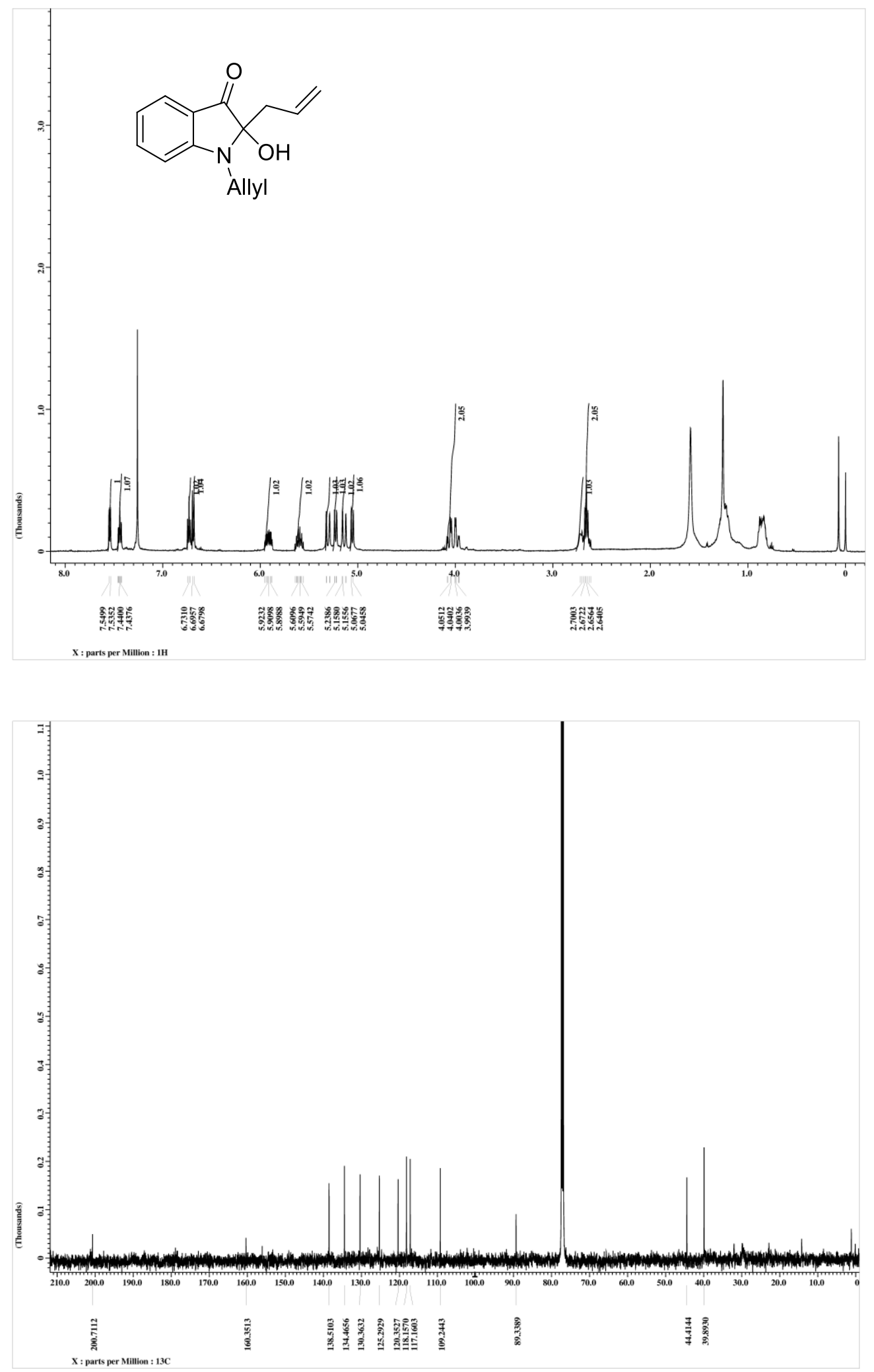
${ }^{1} \mathrm{H}$ and ${ }^{13} \mathrm{C}$ NMR spectra of $\mathbf{3 s}$ :
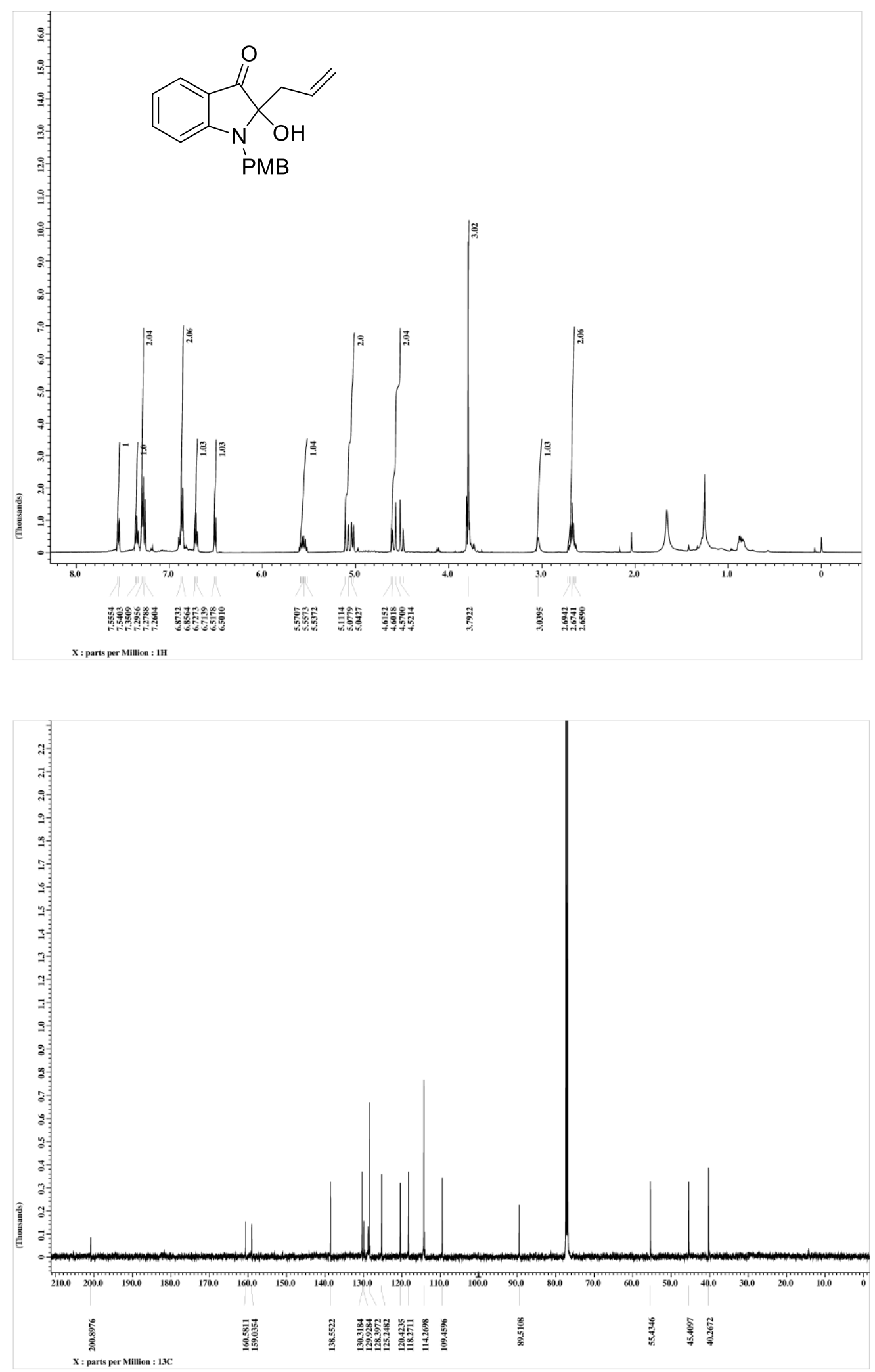
${ }^{1} \mathrm{H}$ and ${ }^{13} \mathrm{C}$ NMR spectra of $\mathbf{3 t}$ :
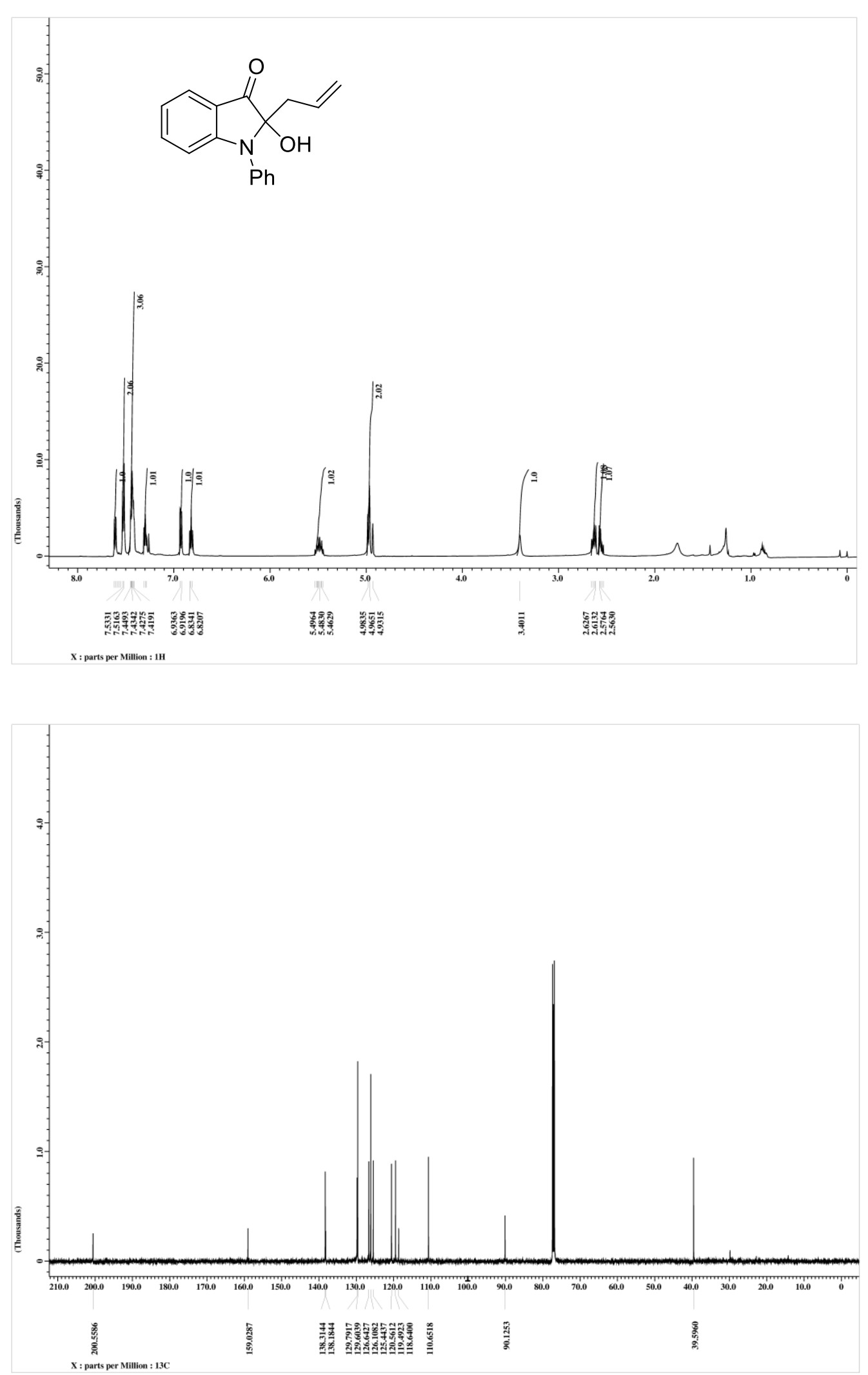
${ }^{1} \mathrm{H}$ and ${ }^{13} \mathrm{C}$ NMR spectra of $\mathbf{3 u}$ :
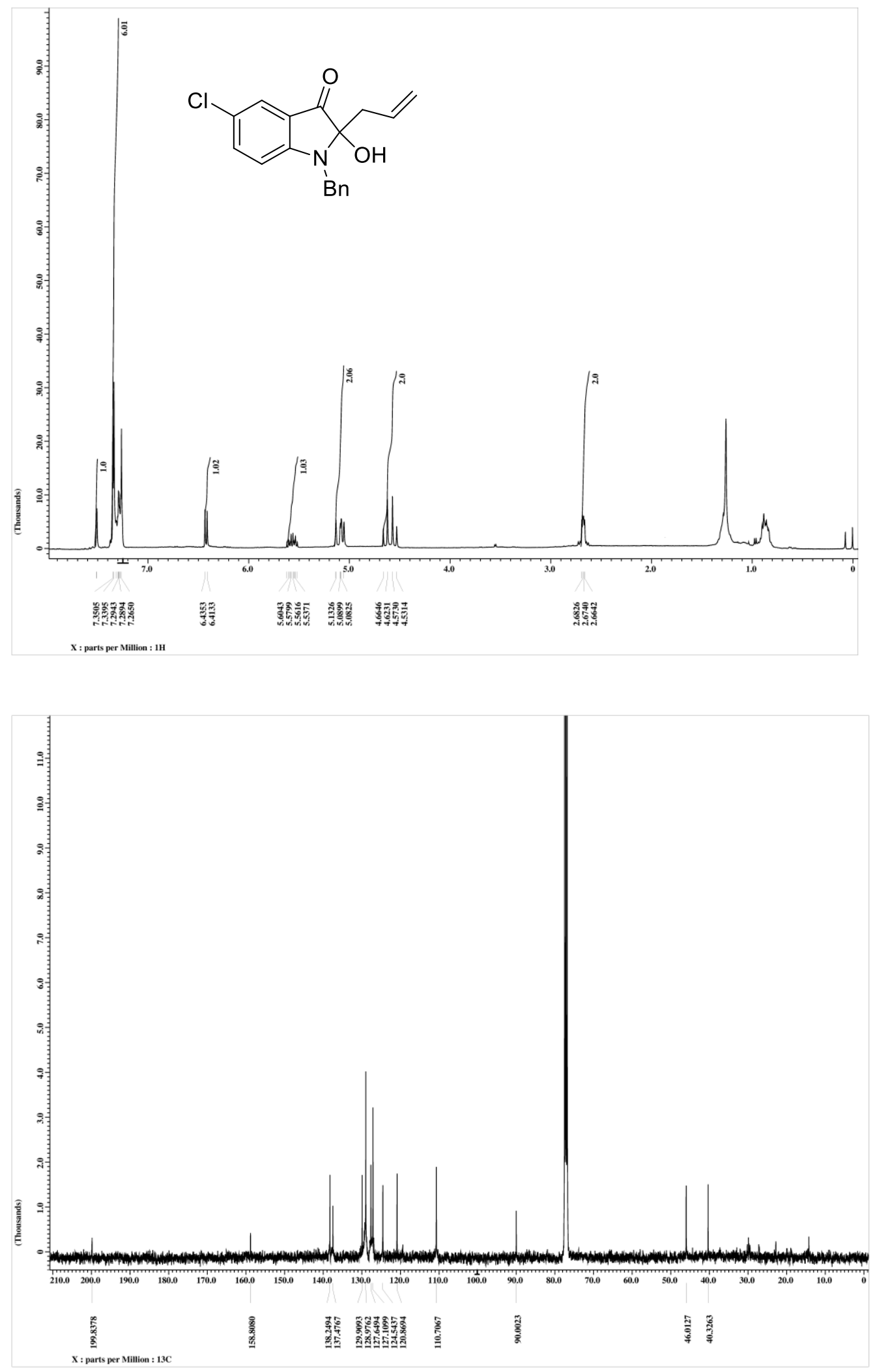
${ }^{1} \mathrm{H}$ and ${ }^{13} \mathrm{C}$ NMR spectra of $\mathbf{3 v}$ :
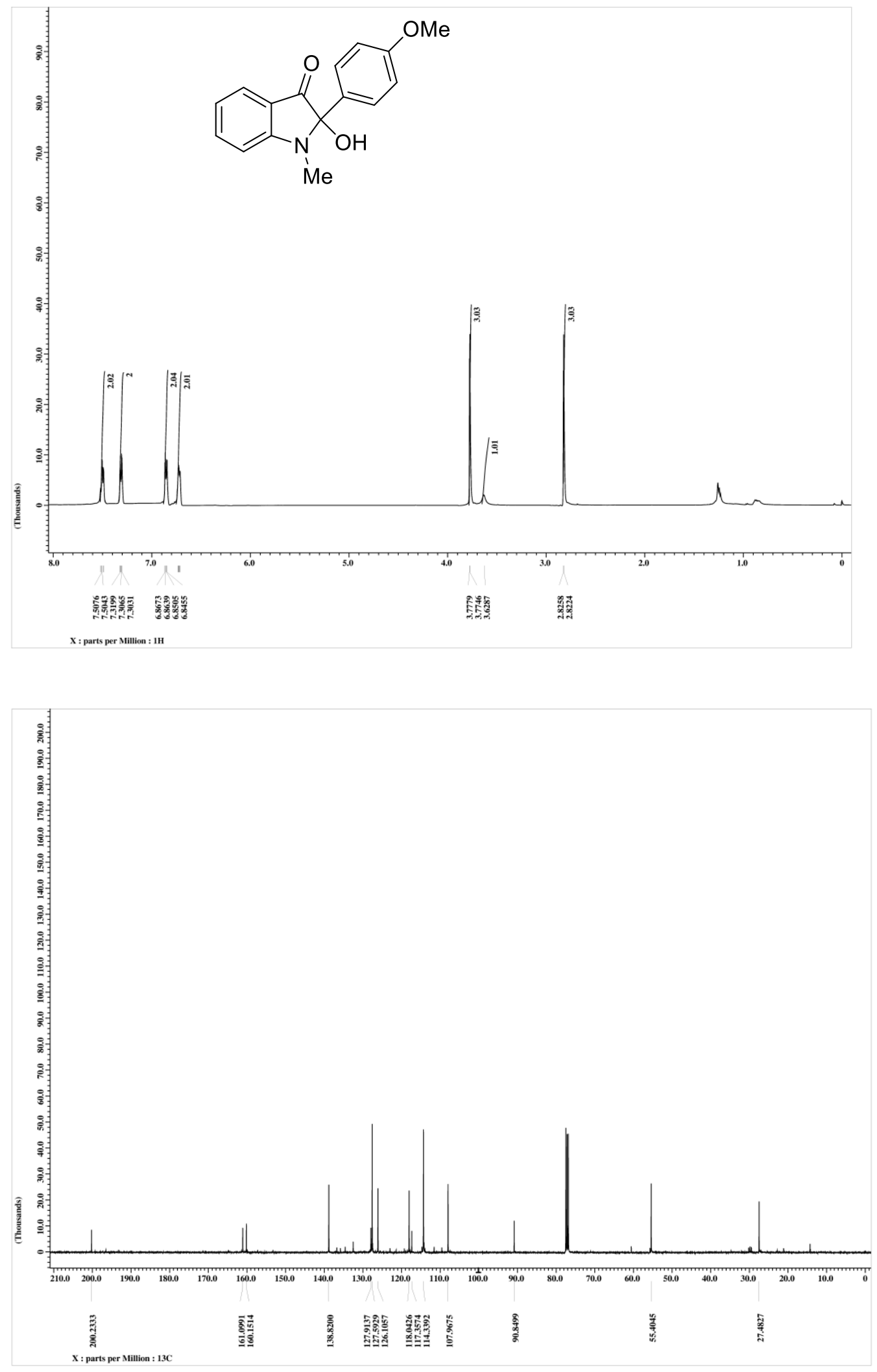
${ }^{1} \mathrm{H}$ and ${ }^{13} \mathrm{C}$ NMR spectra of $\mathbf{3 w}$ :
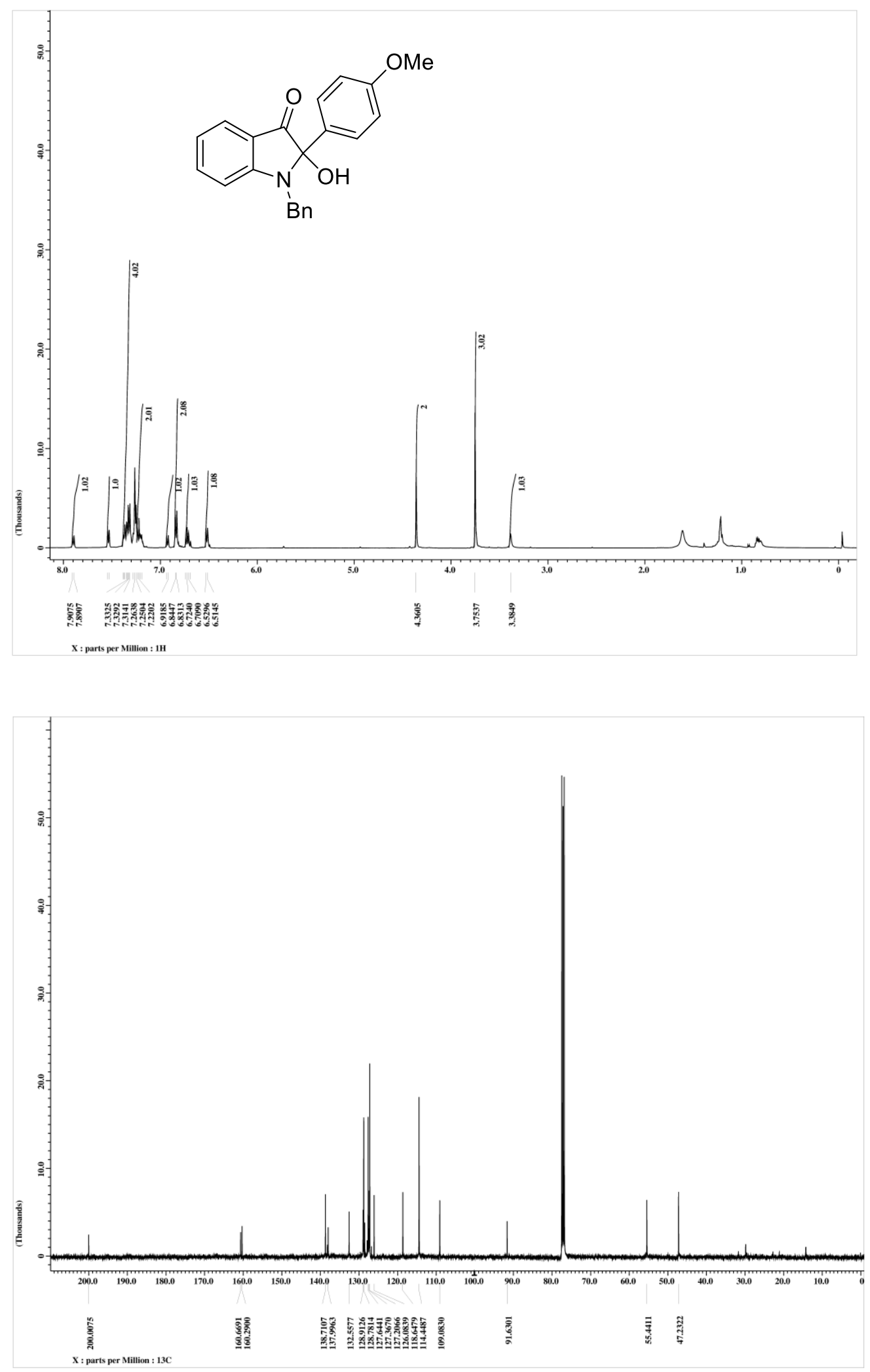
${ }^{1} \mathrm{H}$ and ${ }^{13} \mathrm{C}$ NMR spectra of $\mathbf{3 x}$ :
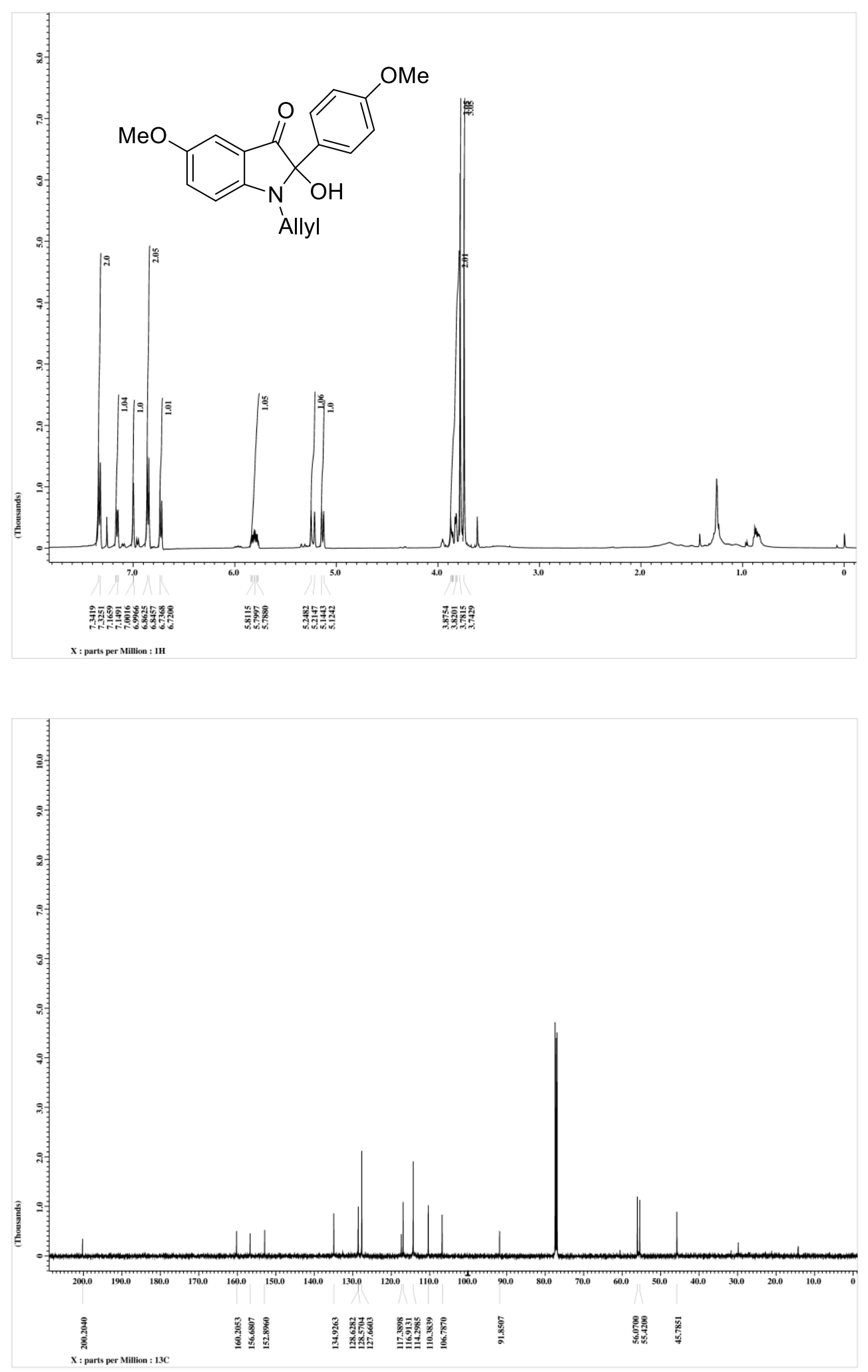
${ }^{1} \mathrm{H}$ and ${ }^{13} \mathrm{C}$ NMR spectra of $\mathbf{3 y}$ :
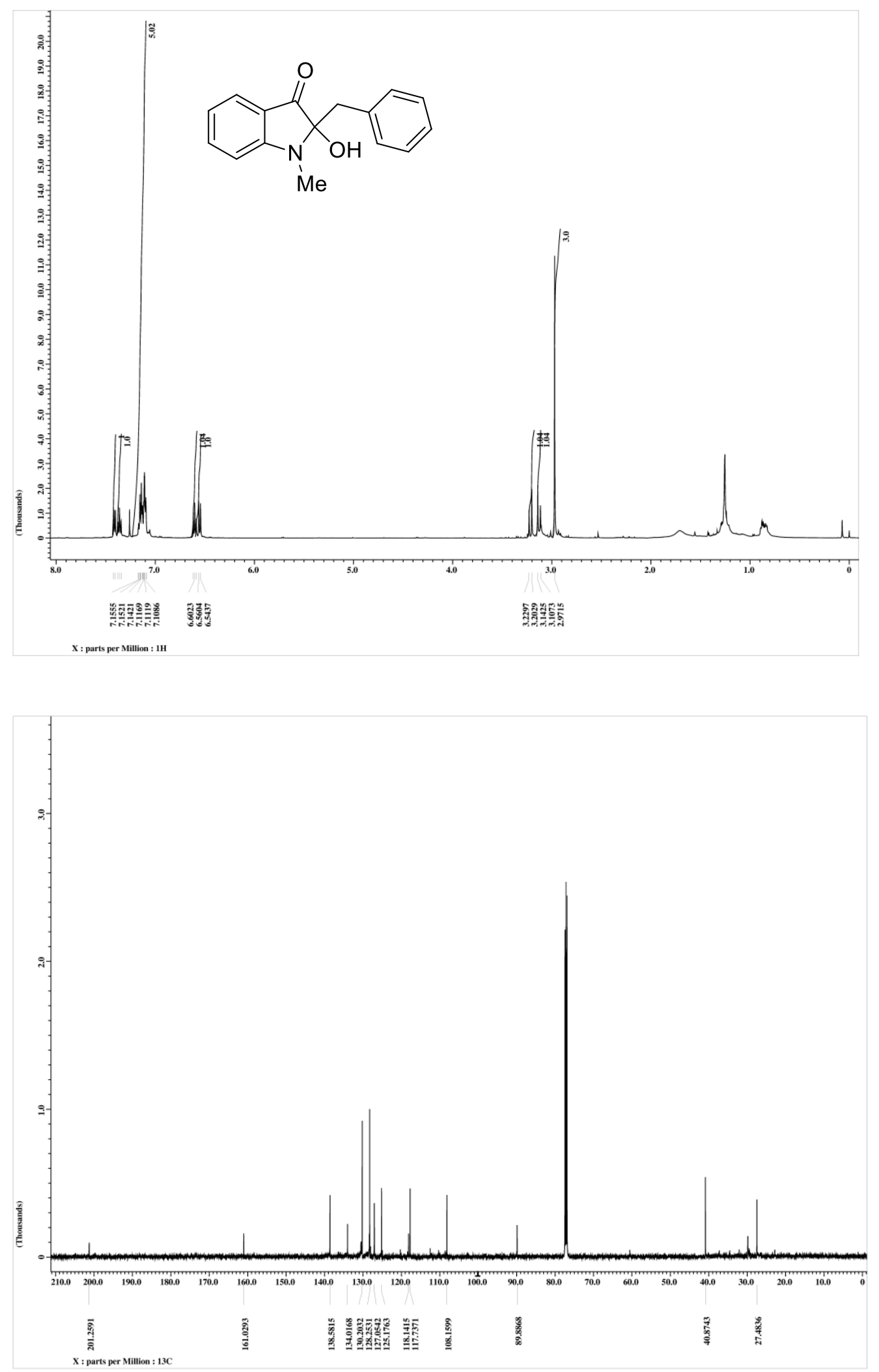
${ }^{1} \mathrm{H}$ and ${ }^{13} \mathrm{C}$ NMR spectra of $\mathbf{3 z}$ :
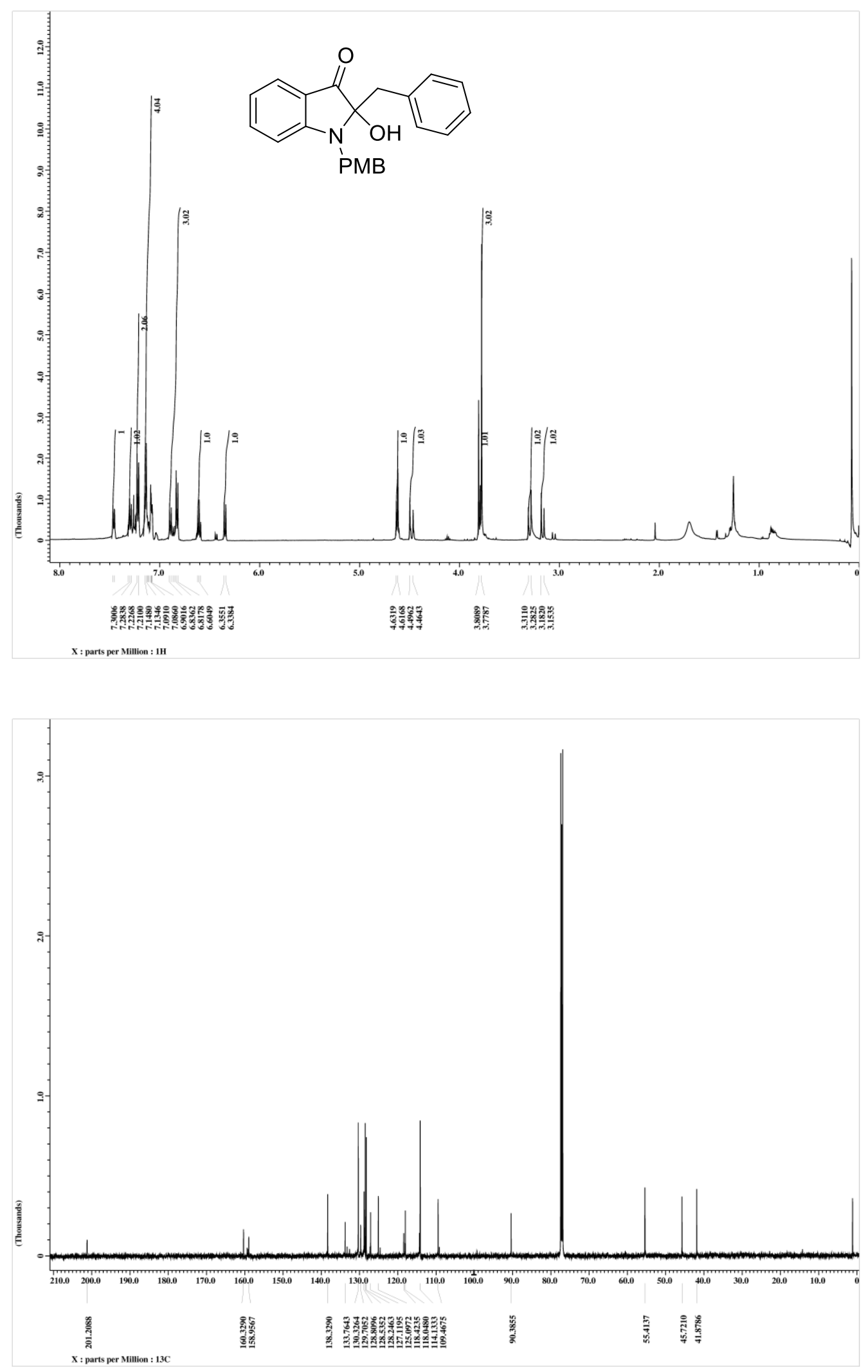
${ }^{1} \mathrm{H}$ and ${ }^{13} \mathrm{C}$ NMR spectra of 3a':
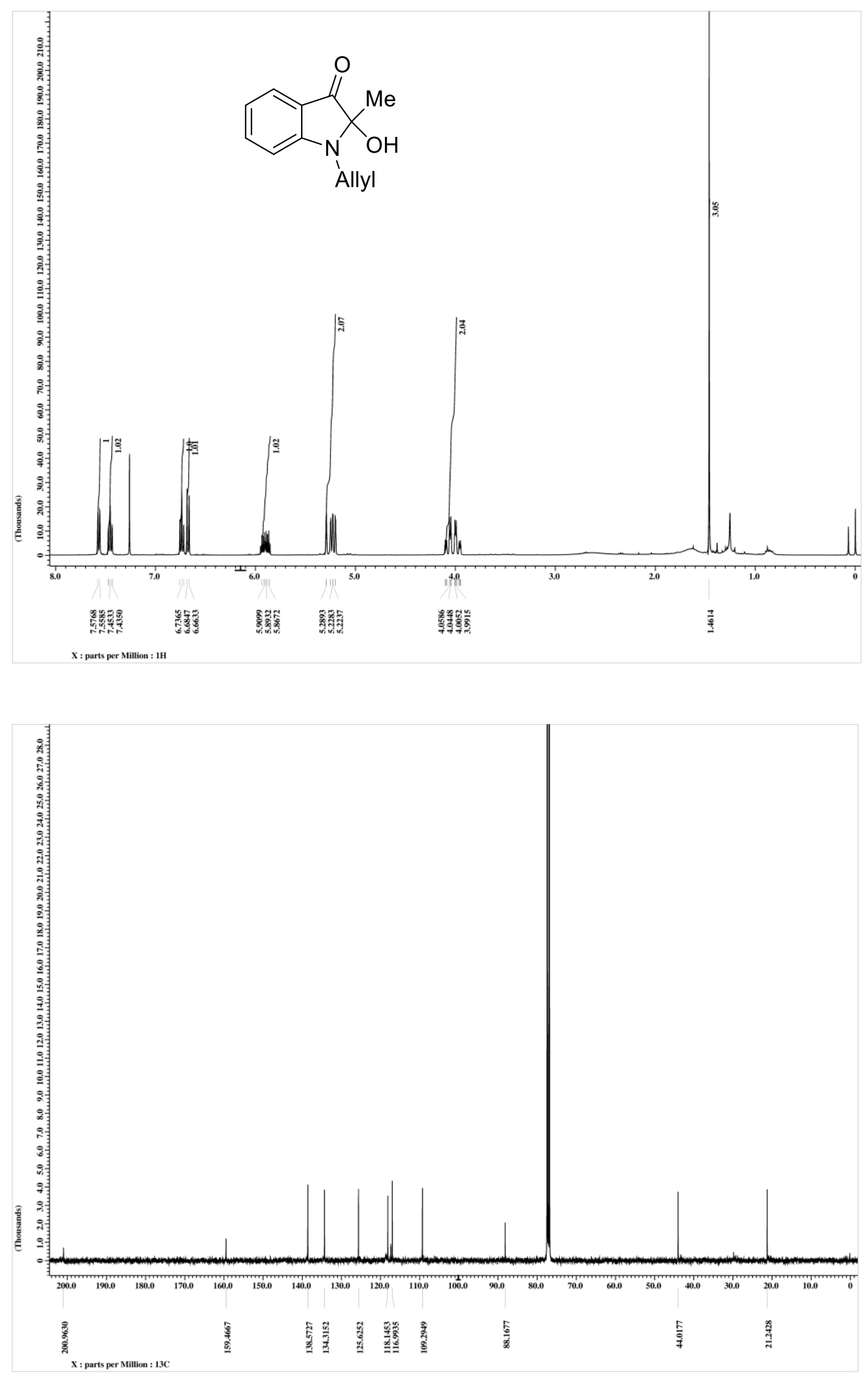
${ }^{1} \mathrm{H}$ and ${ }^{13} \mathrm{C}$ NMR spectra of $\mathbf{3} \mathbf{b}$ ':
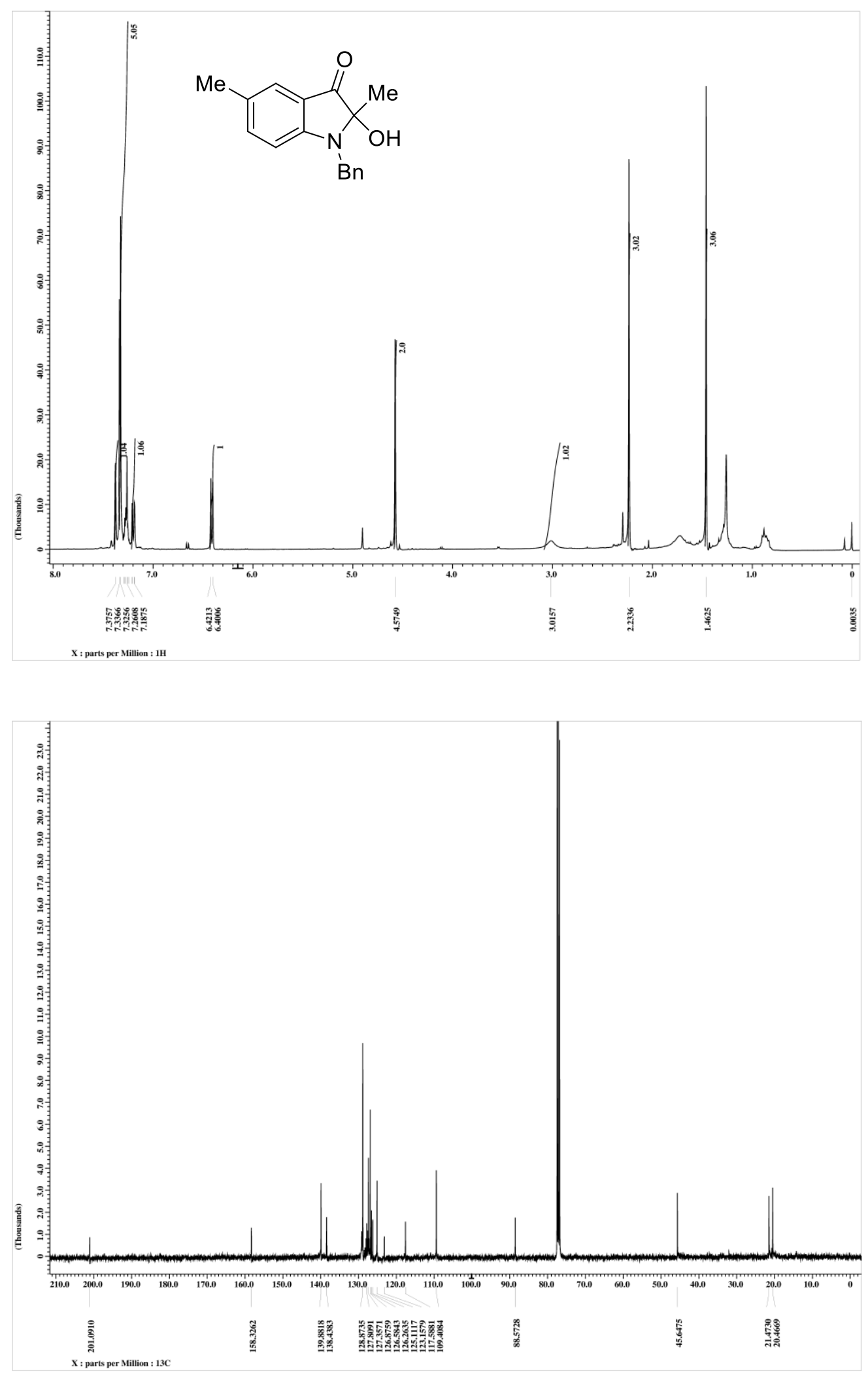
${ }^{1} \mathrm{H}$ and ${ }^{13} \mathrm{C}$ NMR spectra of $\mathbf{3} \mathbf{c}^{\prime}$ :
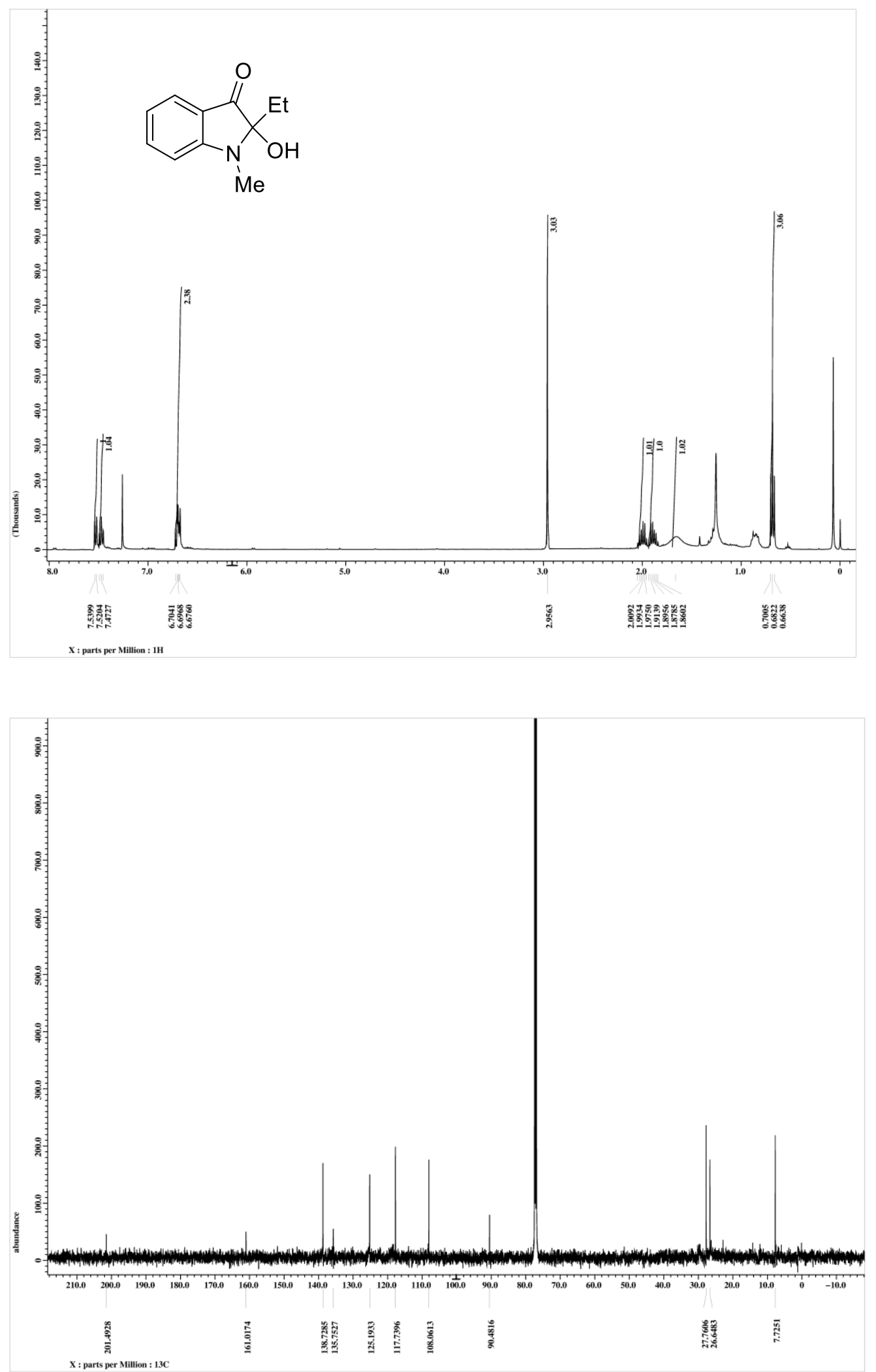
${ }^{1} \mathrm{H}$ and ${ }^{13} \mathrm{C}$ NMR spectra of $\mathbf{3 d}$ ':
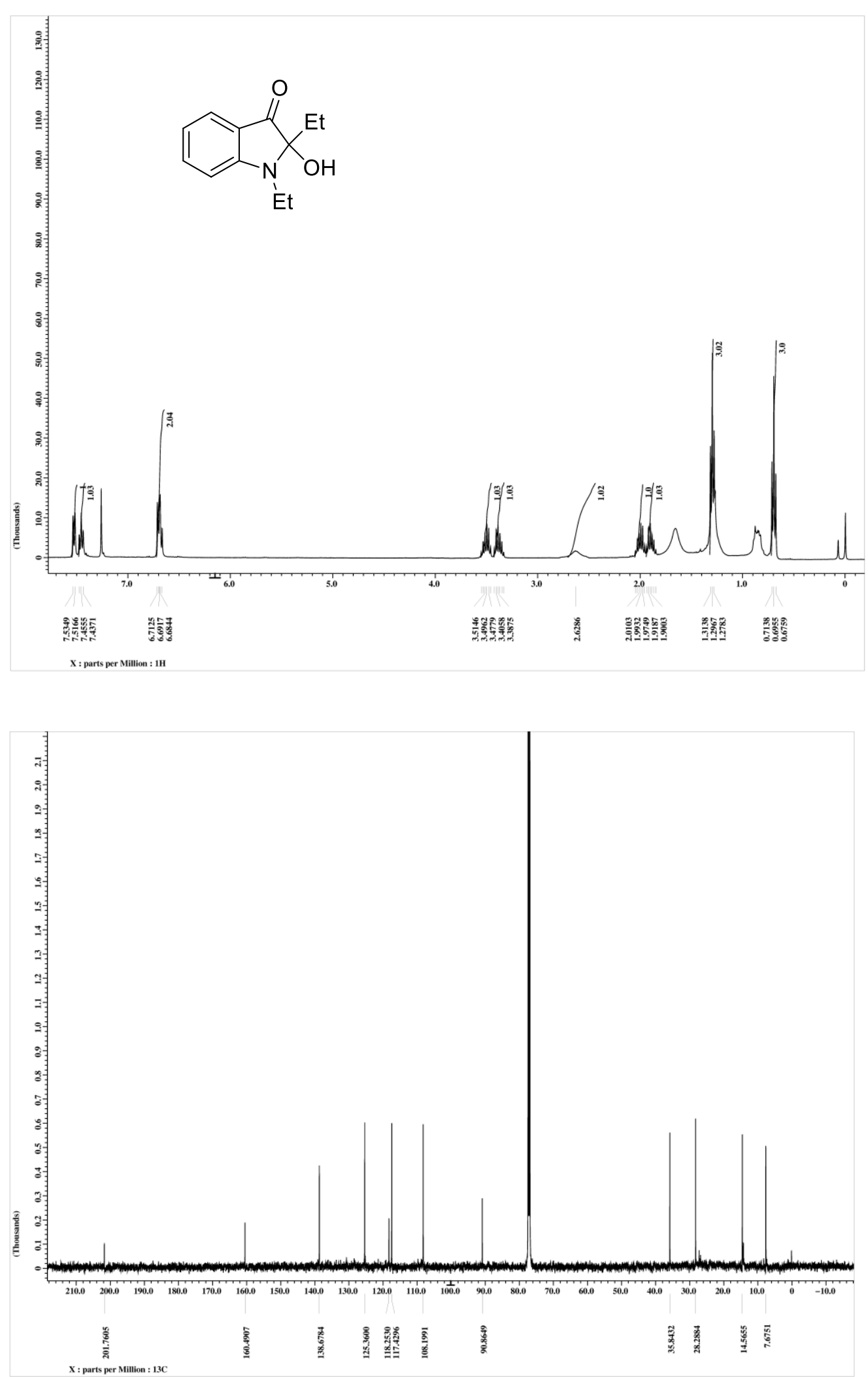
${ }^{1} \mathrm{H}$ and ${ }^{13} \mathrm{C}$ NMR spectra of $3 \mathrm{e}^{\mathrm{\prime}}$ :
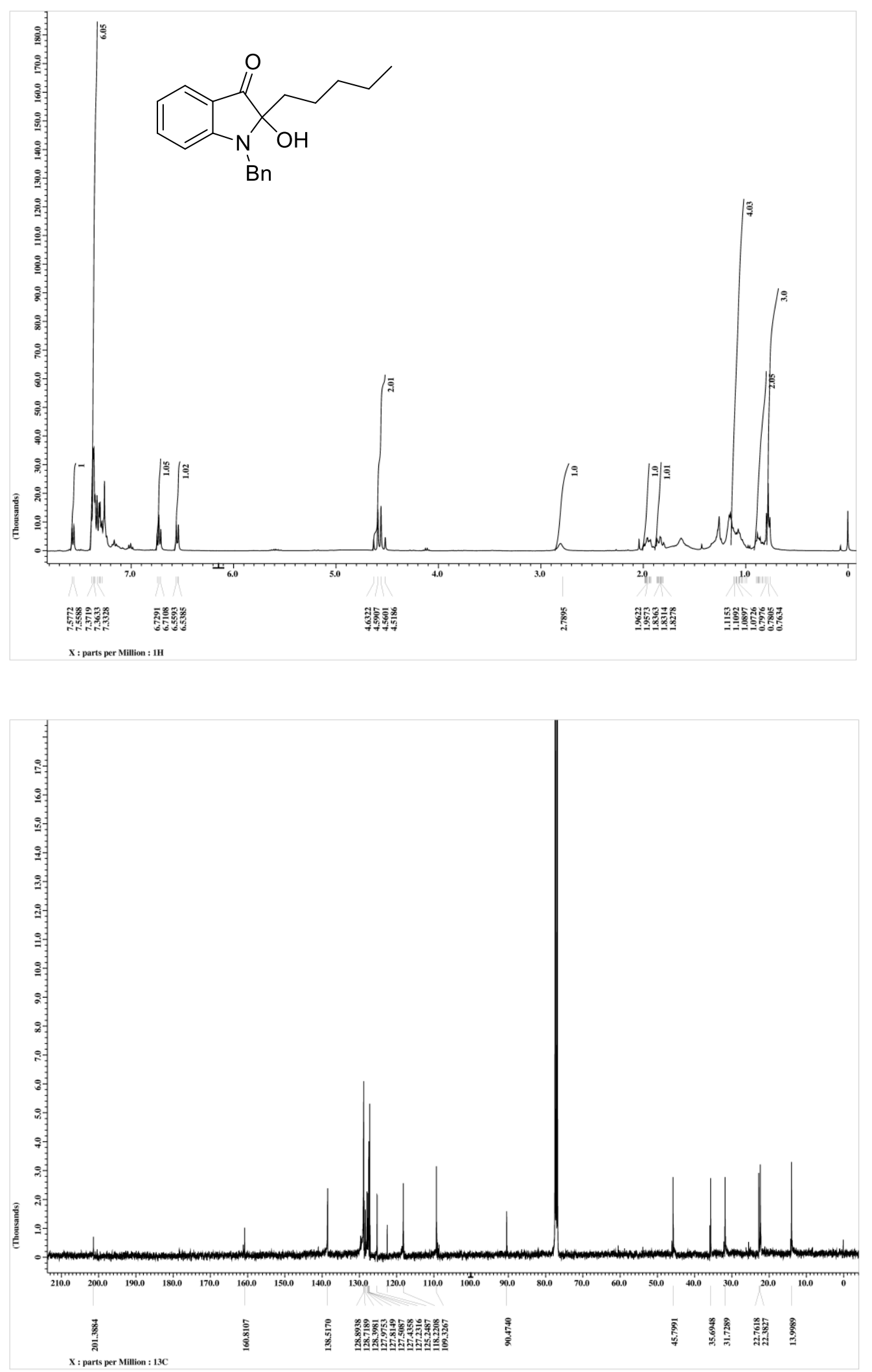
${ }^{1} \mathrm{H}$ and ${ }^{13} \mathrm{C}$ NMR spectra of $\mathbf{3 f}$ ':
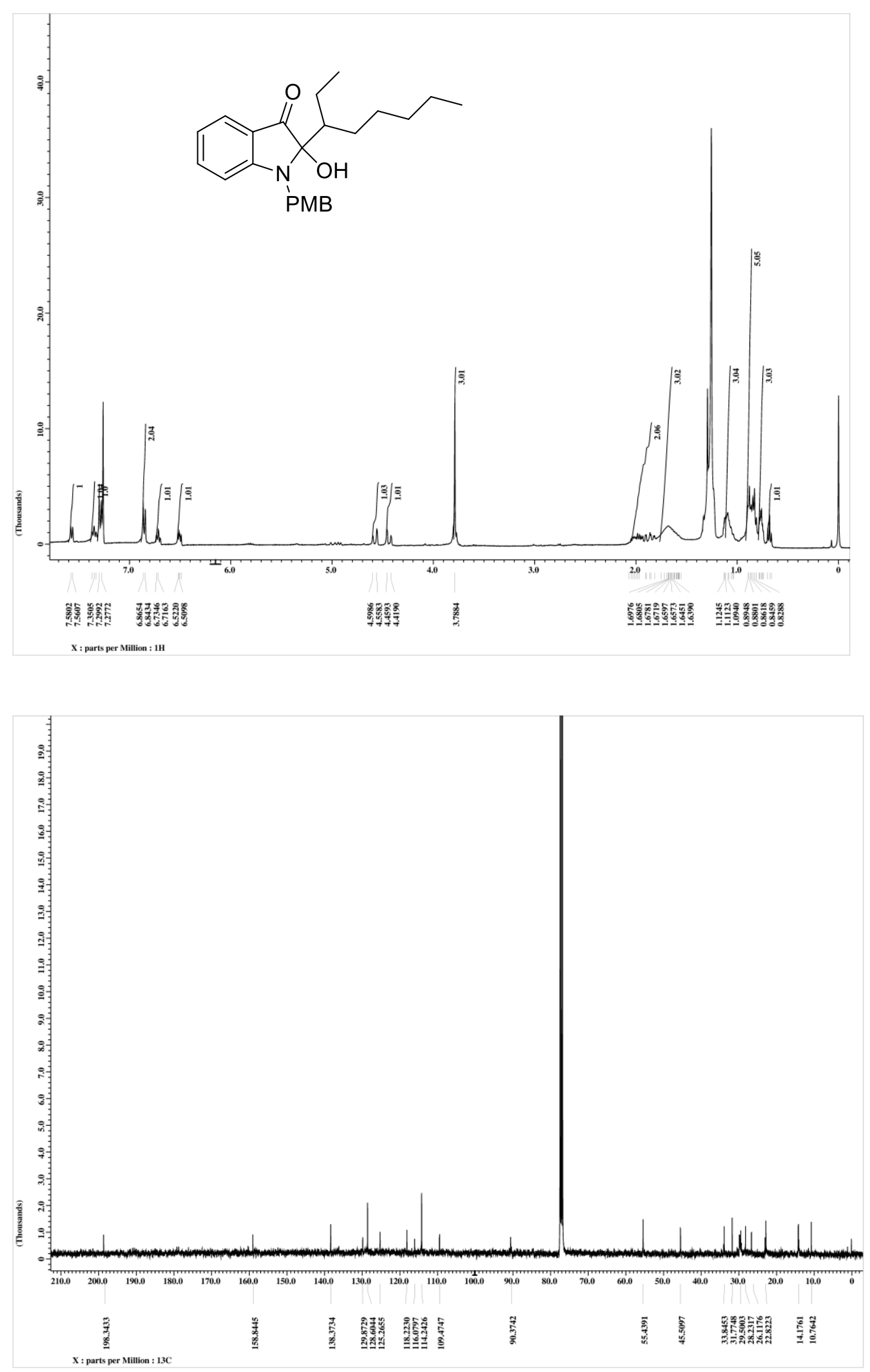
${ }^{1} \mathrm{H}$ and ${ }^{13} \mathrm{C}$ NMR spectra of $3 \mathbf{g}^{\prime}$ :
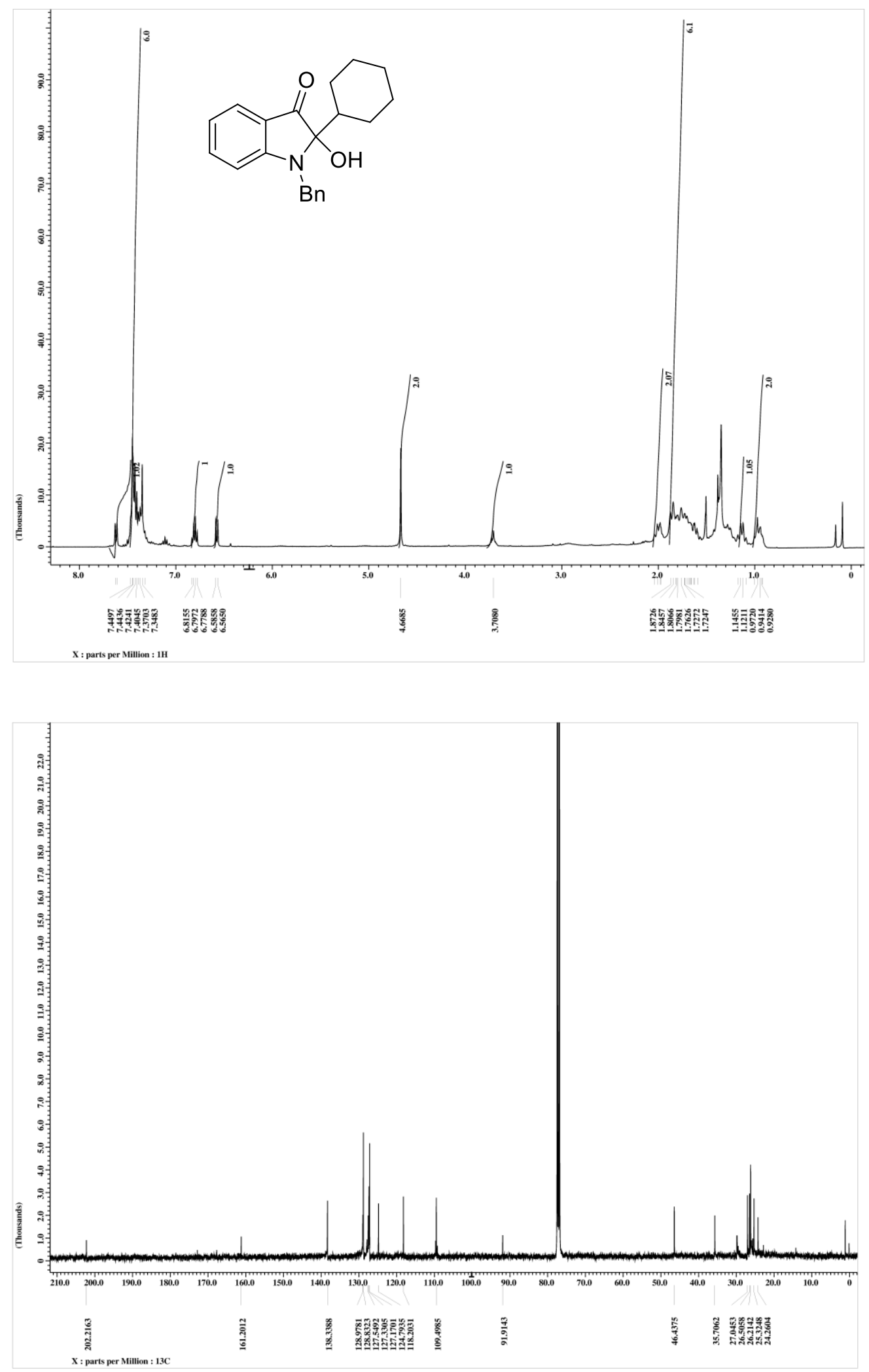
${ }^{1} \mathrm{H}$ and ${ }^{13} \mathrm{C}$ NMR spectra of $6 a$ :
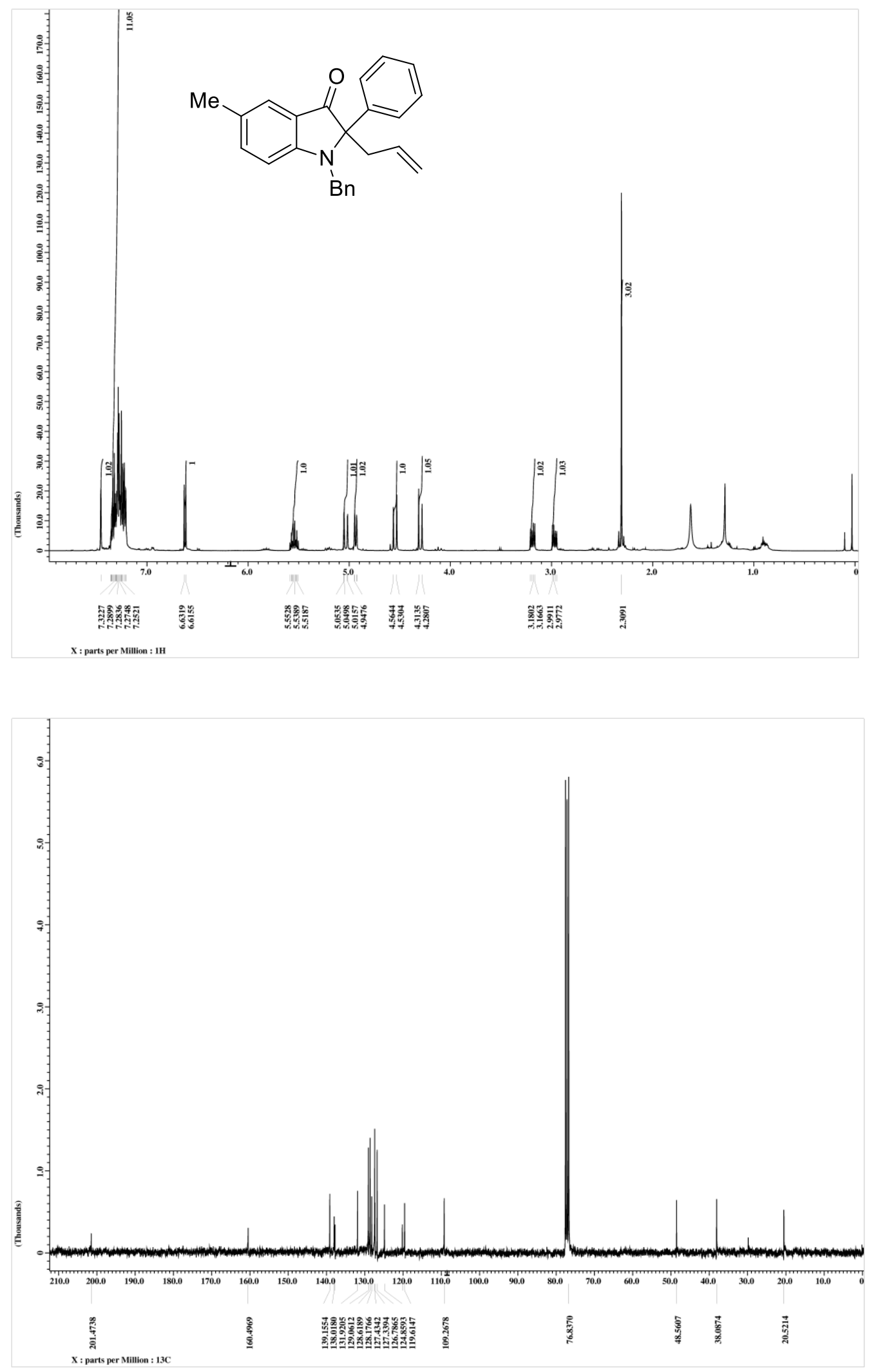
${ }^{1} \mathrm{H}$ and ${ }^{13} \mathrm{C}$ NMR spectra of $\mathbf{6 b}$
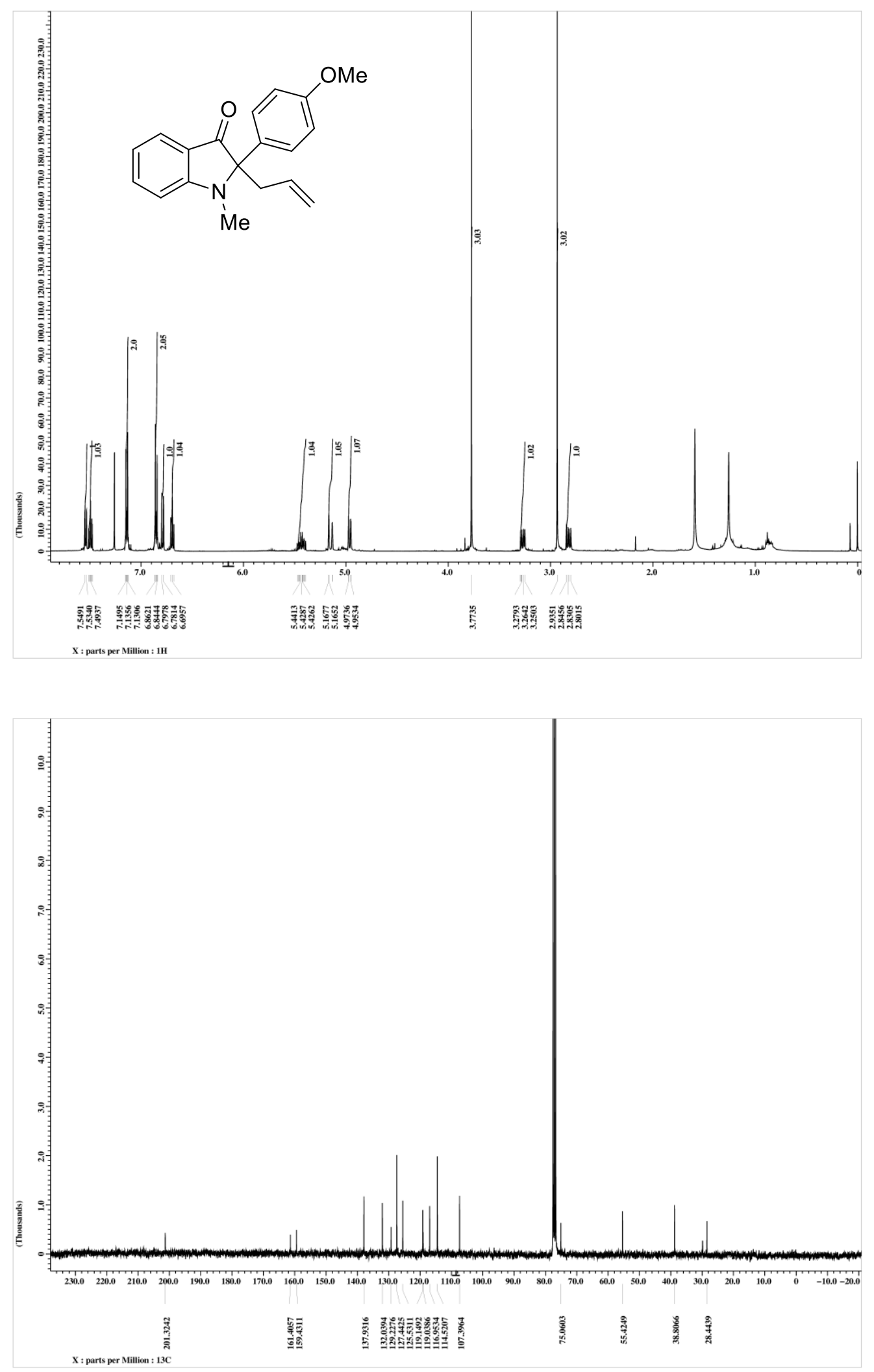
${ }^{1} \mathrm{H}$ and ${ }^{13} \mathrm{C}$ NMR spectra of 7a:
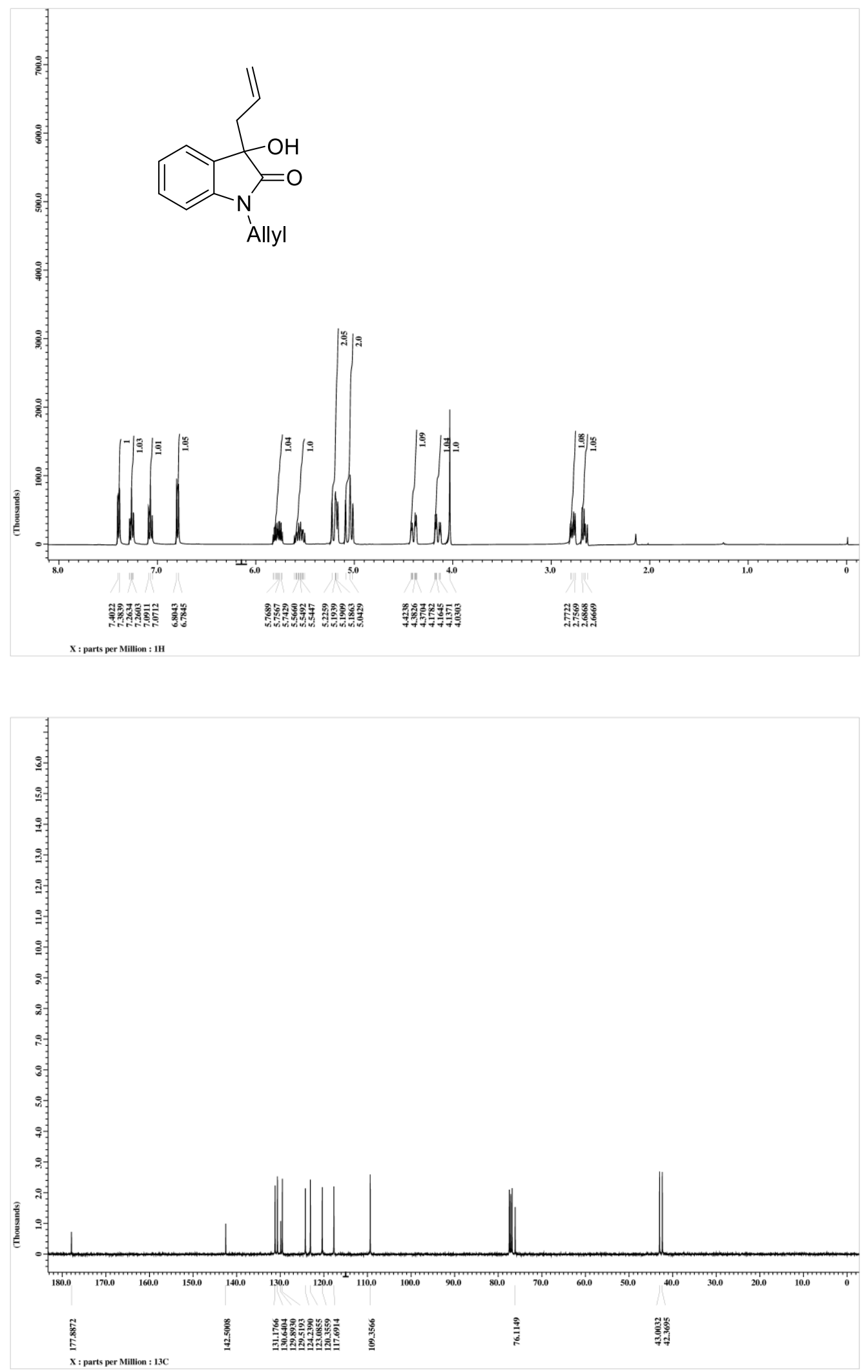
${ }^{1} \mathrm{H}$ and ${ }^{13} \mathrm{C}$ NMR spectra of $\mathbf{7 b}$
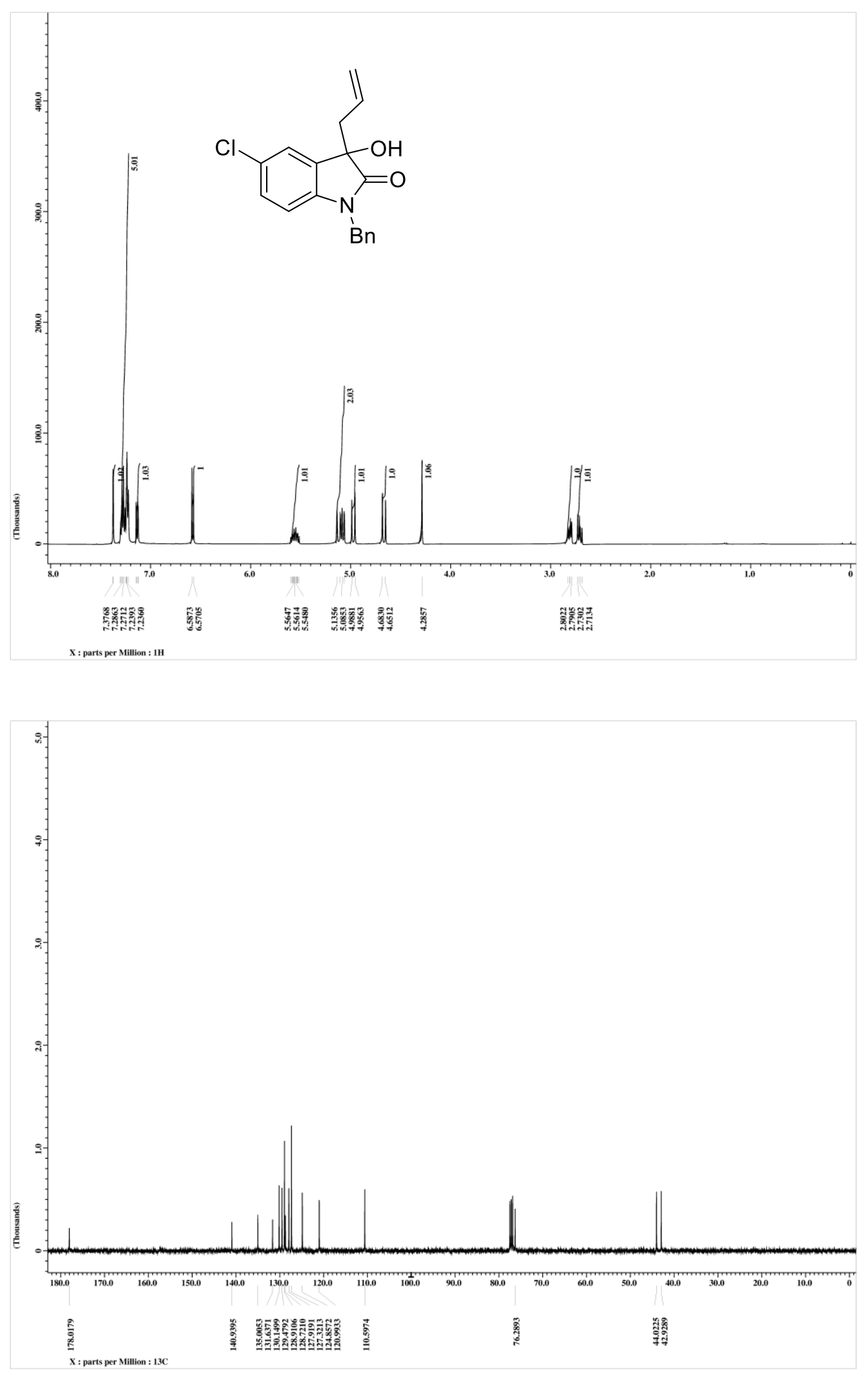
${ }^{1} \mathrm{H}$ and ${ }^{13} \mathrm{C}$ NMR spectra of $7 \mathbf{c}$ :
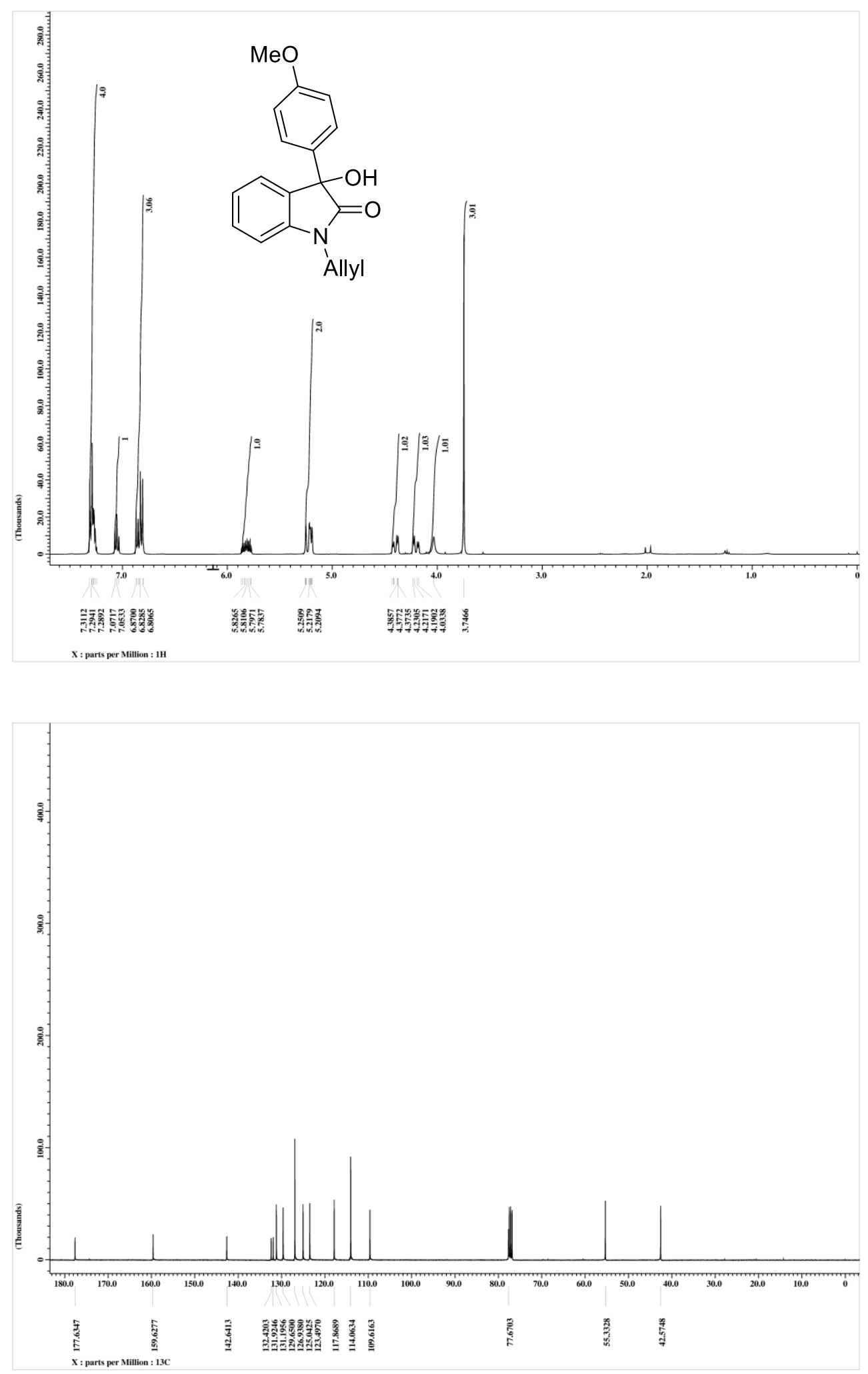
${ }^{1} \mathrm{H}$ and ${ }^{13} \mathrm{C}$ NMR spectra of 7d:
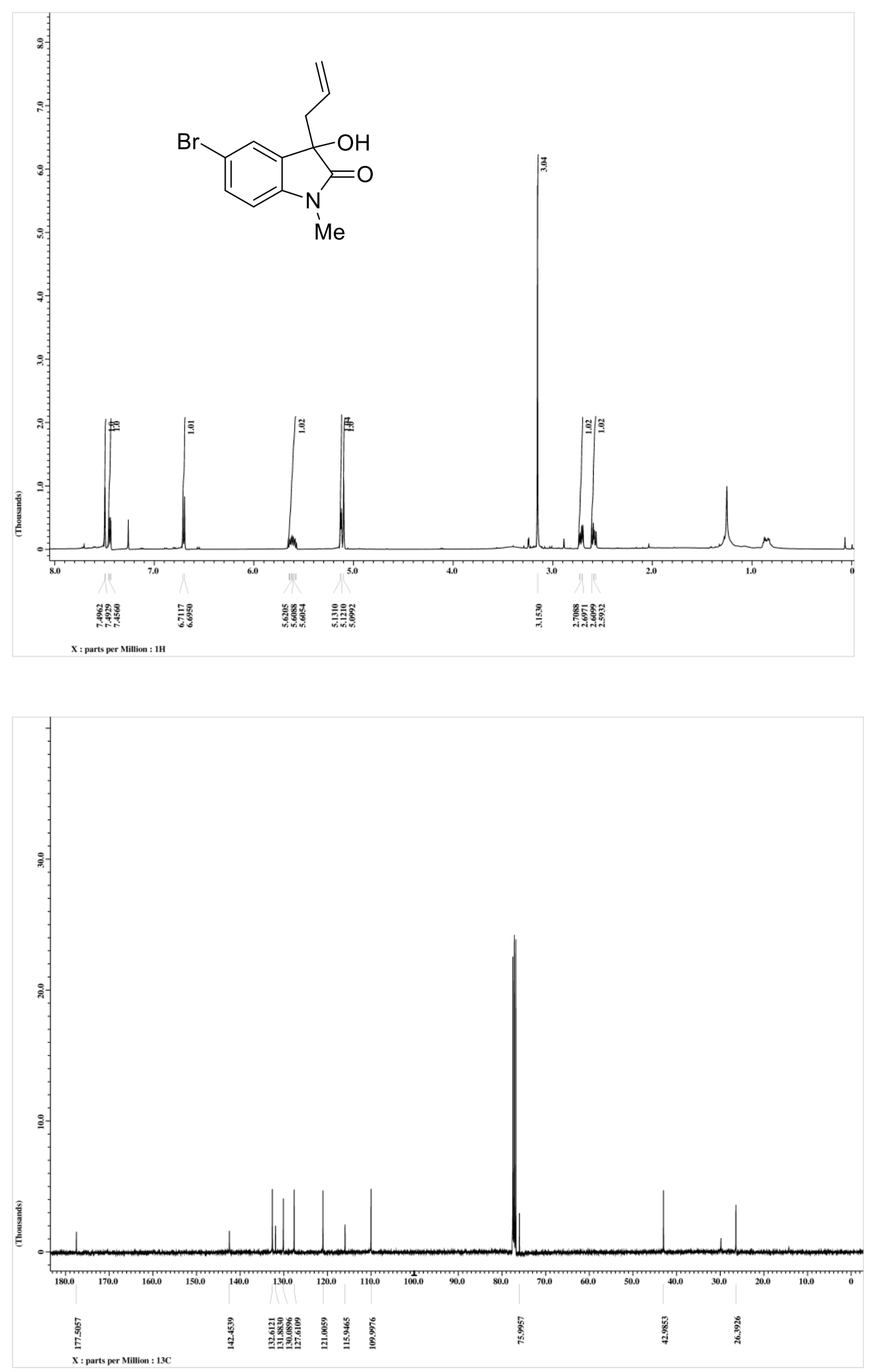
${ }^{1} \mathrm{H}$ and ${ }^{13} \mathrm{C}$ NMR spectra of 7e:
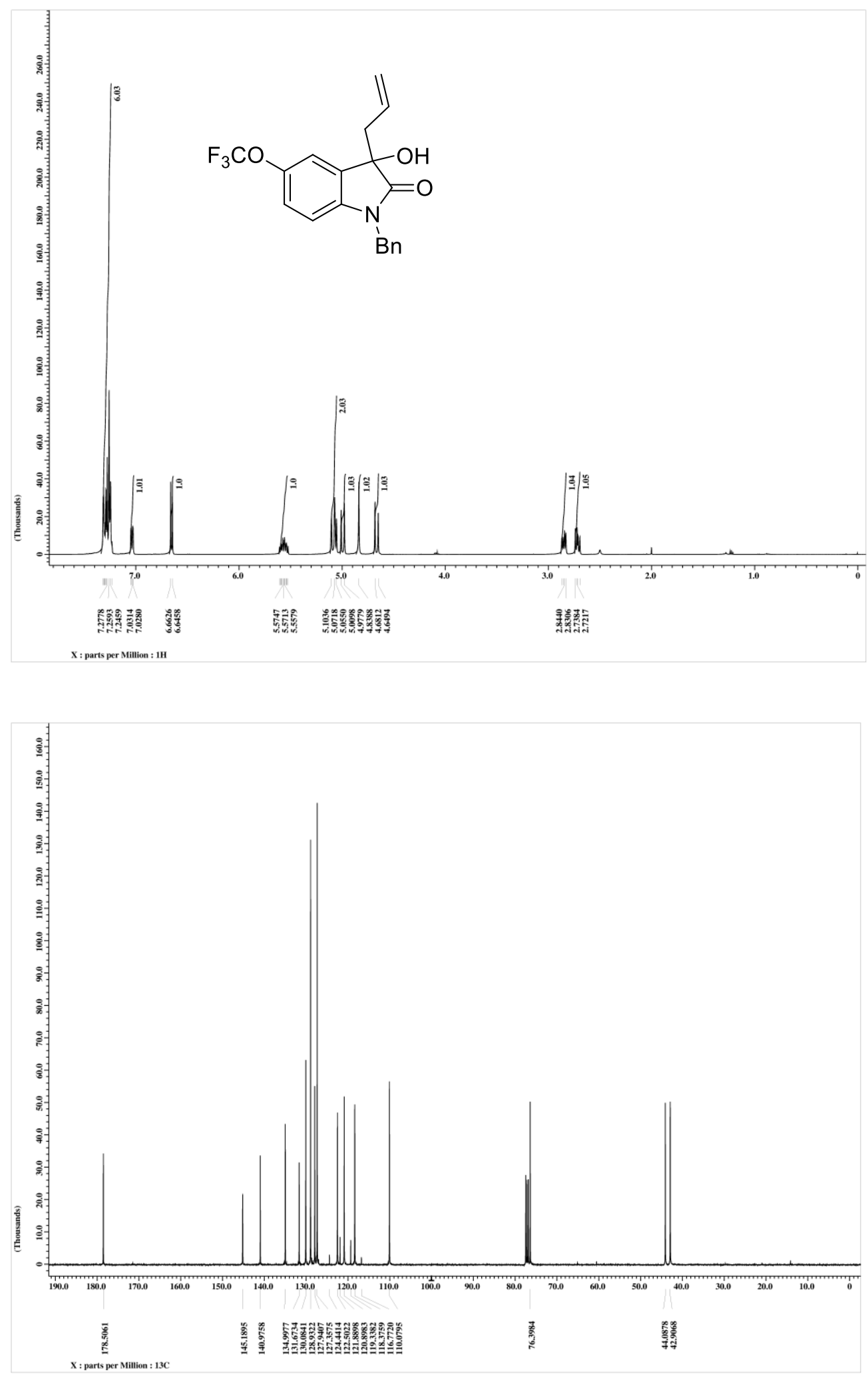
${ }^{1} \mathrm{H}$ and ${ }^{13} \mathrm{C}$ NMR spectra of 8a:
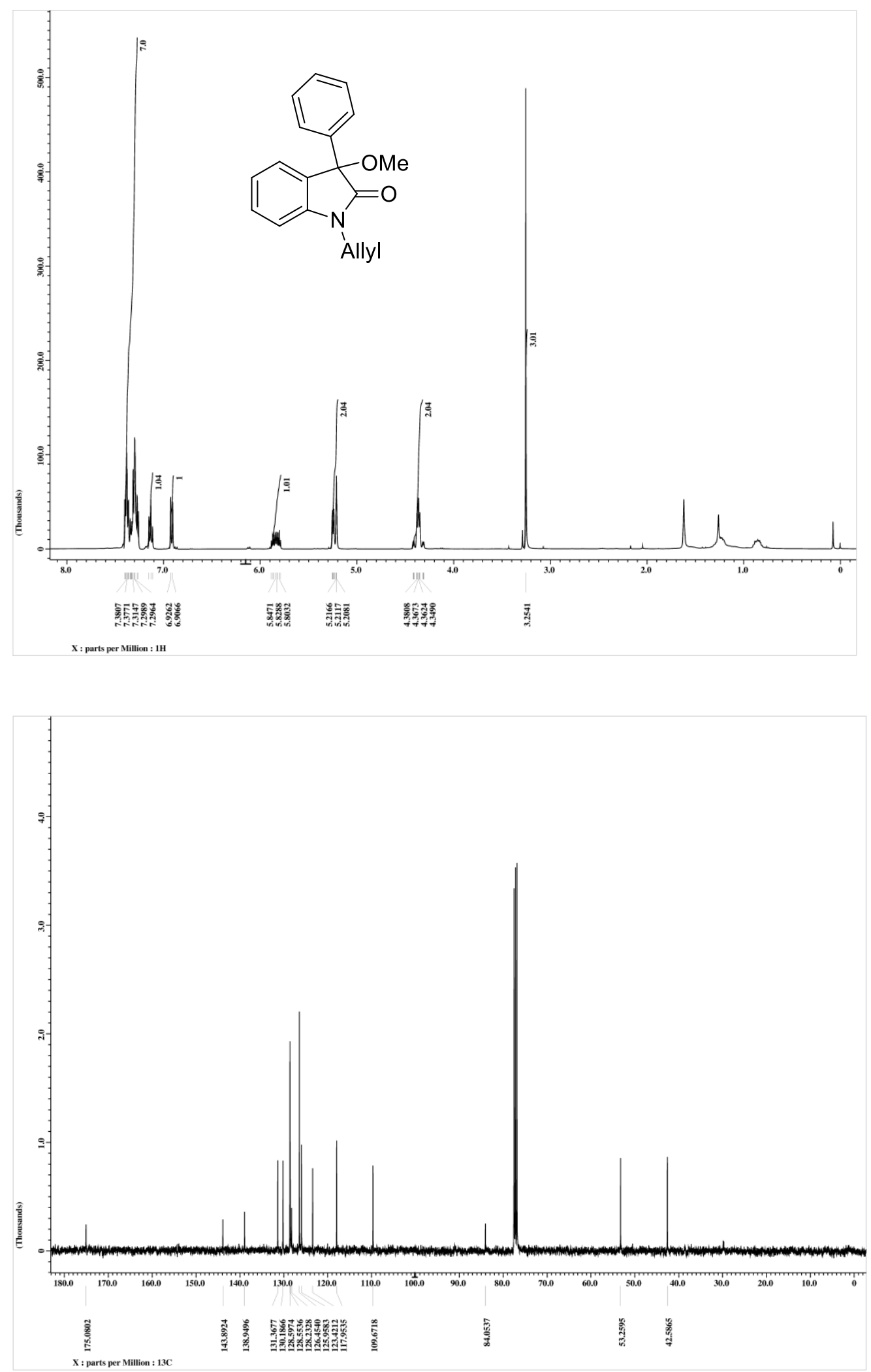
${ }^{1} \mathrm{H}$ and ${ }^{13} \mathrm{C}$ NMR spectra of $\mathbf{8 b}$ :
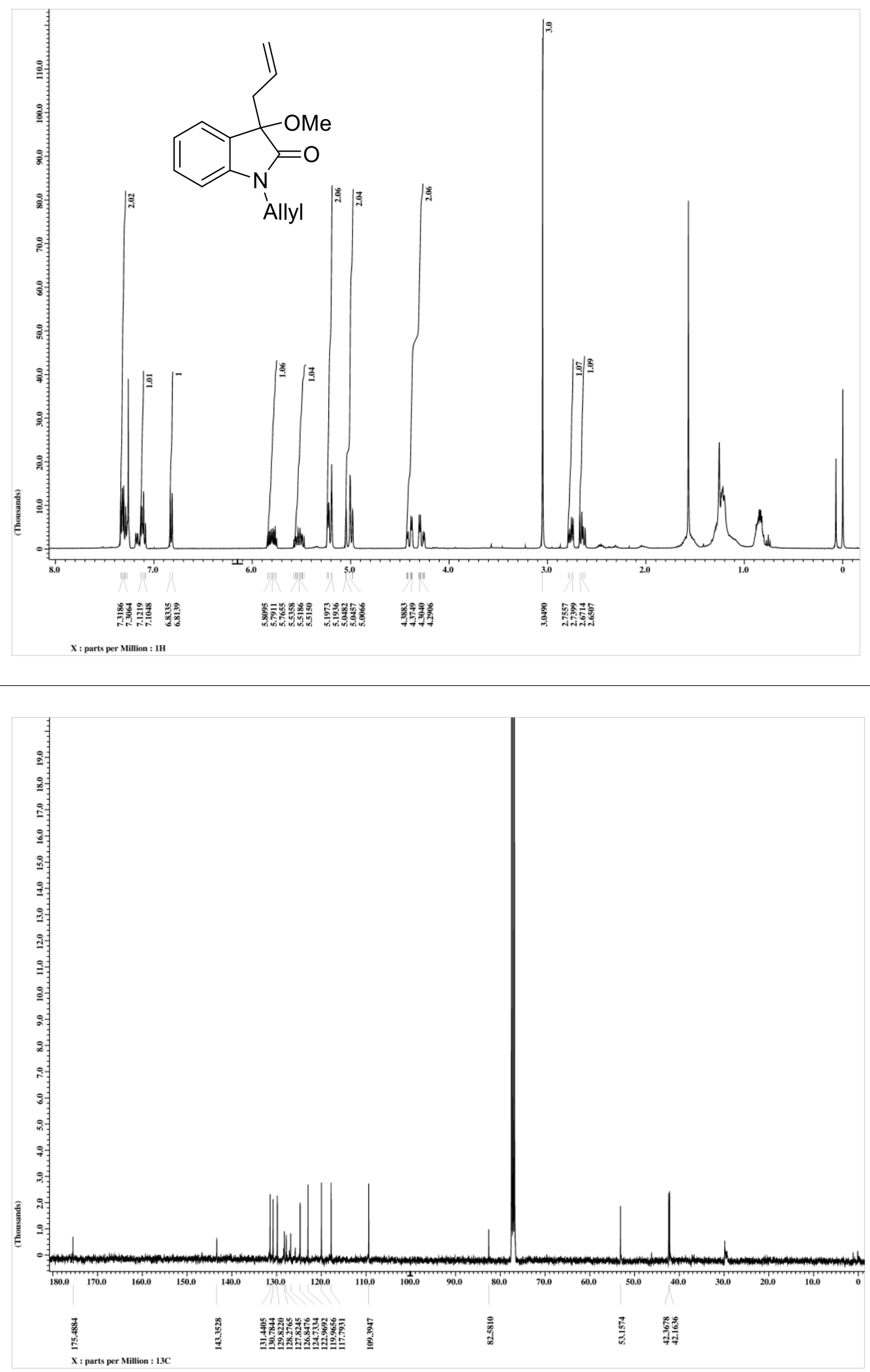
${ }^{1} \mathrm{H}$ and ${ }^{13} \mathrm{C}$ NMR spectra of 9a:
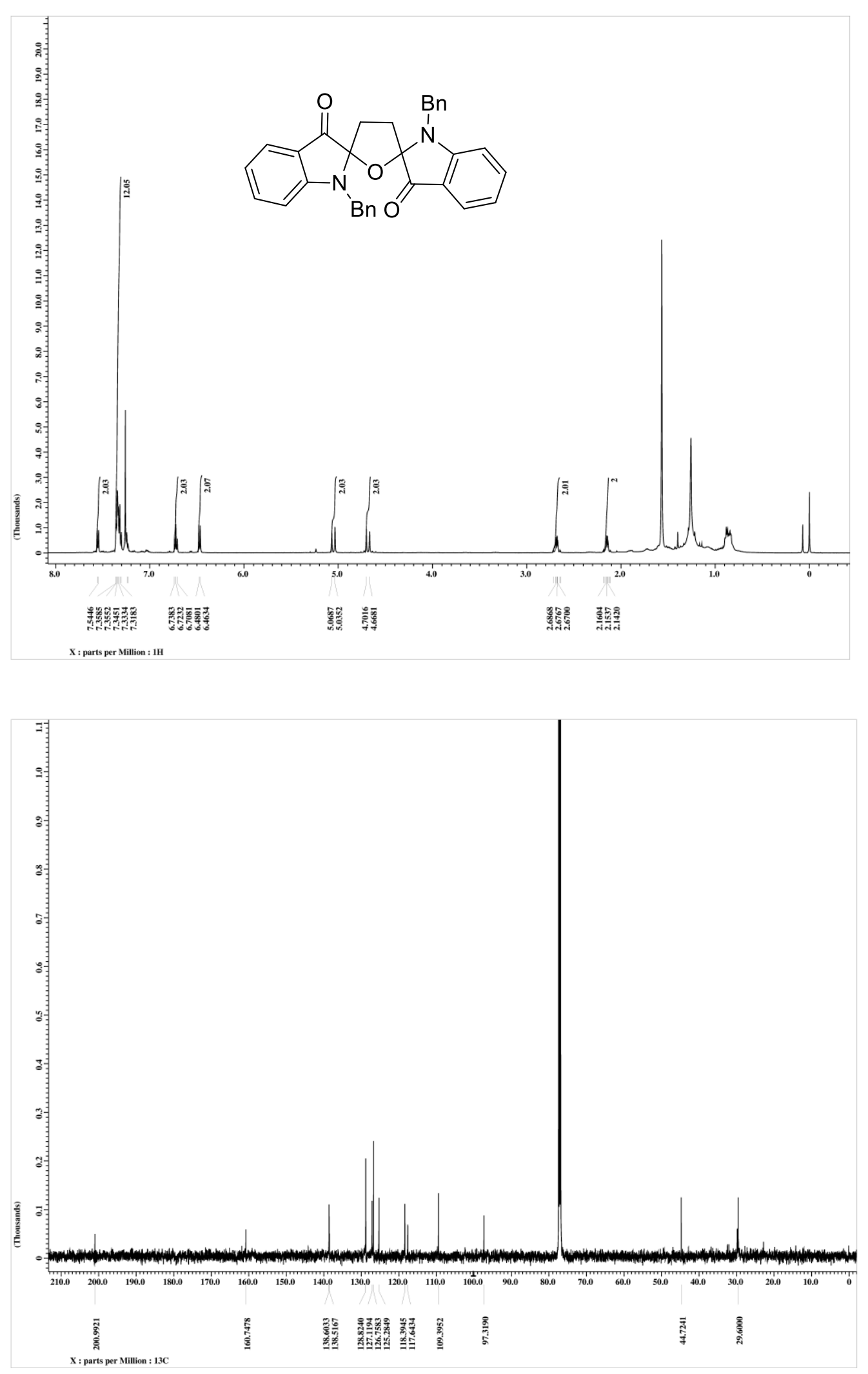
${ }^{1} \mathrm{H}$ and ${ }^{13} \mathrm{C}$ NMR spectra of $9 \mathbf{b}$ :
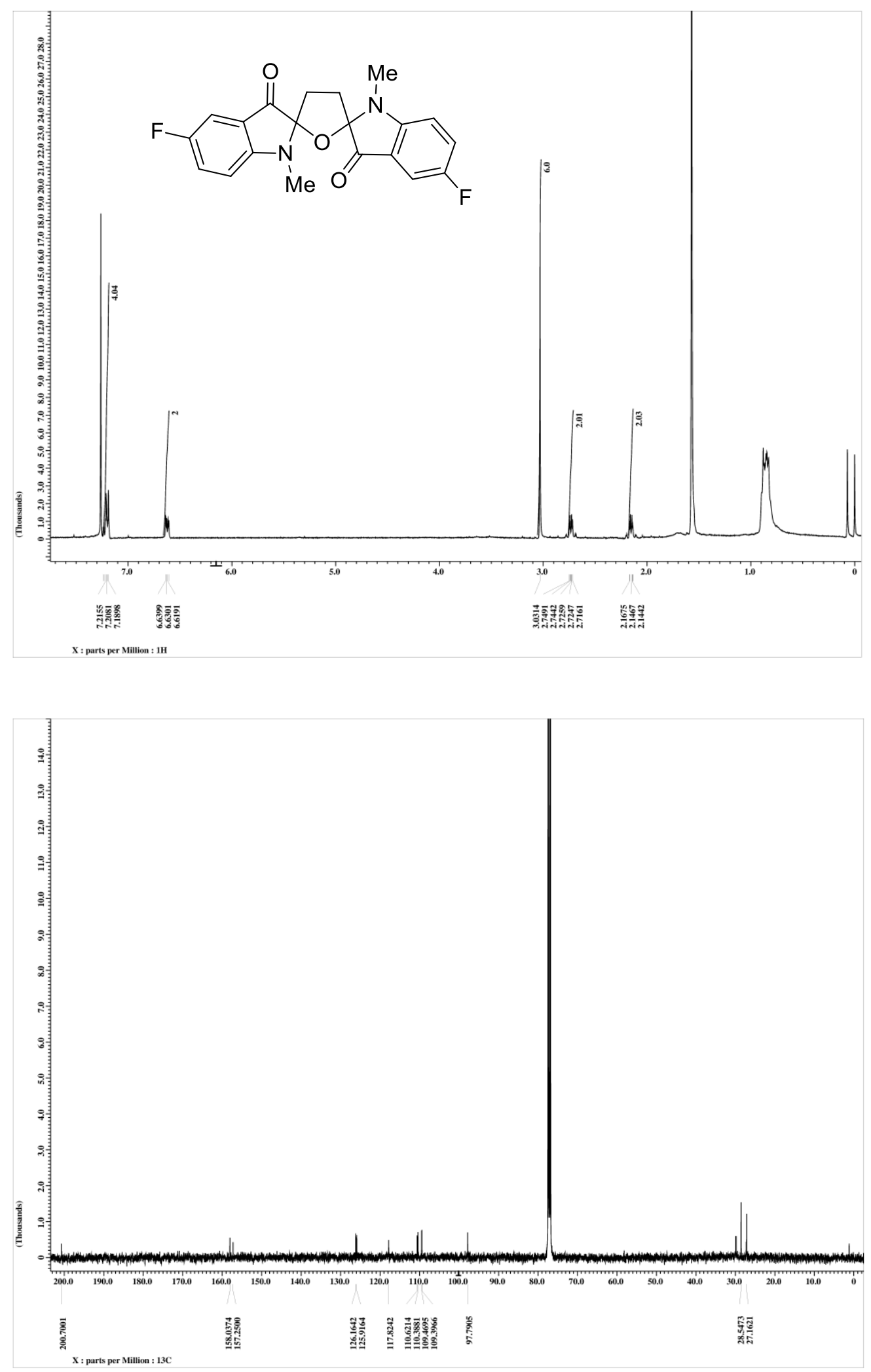
${ }^{1} \mathrm{H}$ and ${ }^{13} \mathrm{C}$ NMR spectra of 9c:
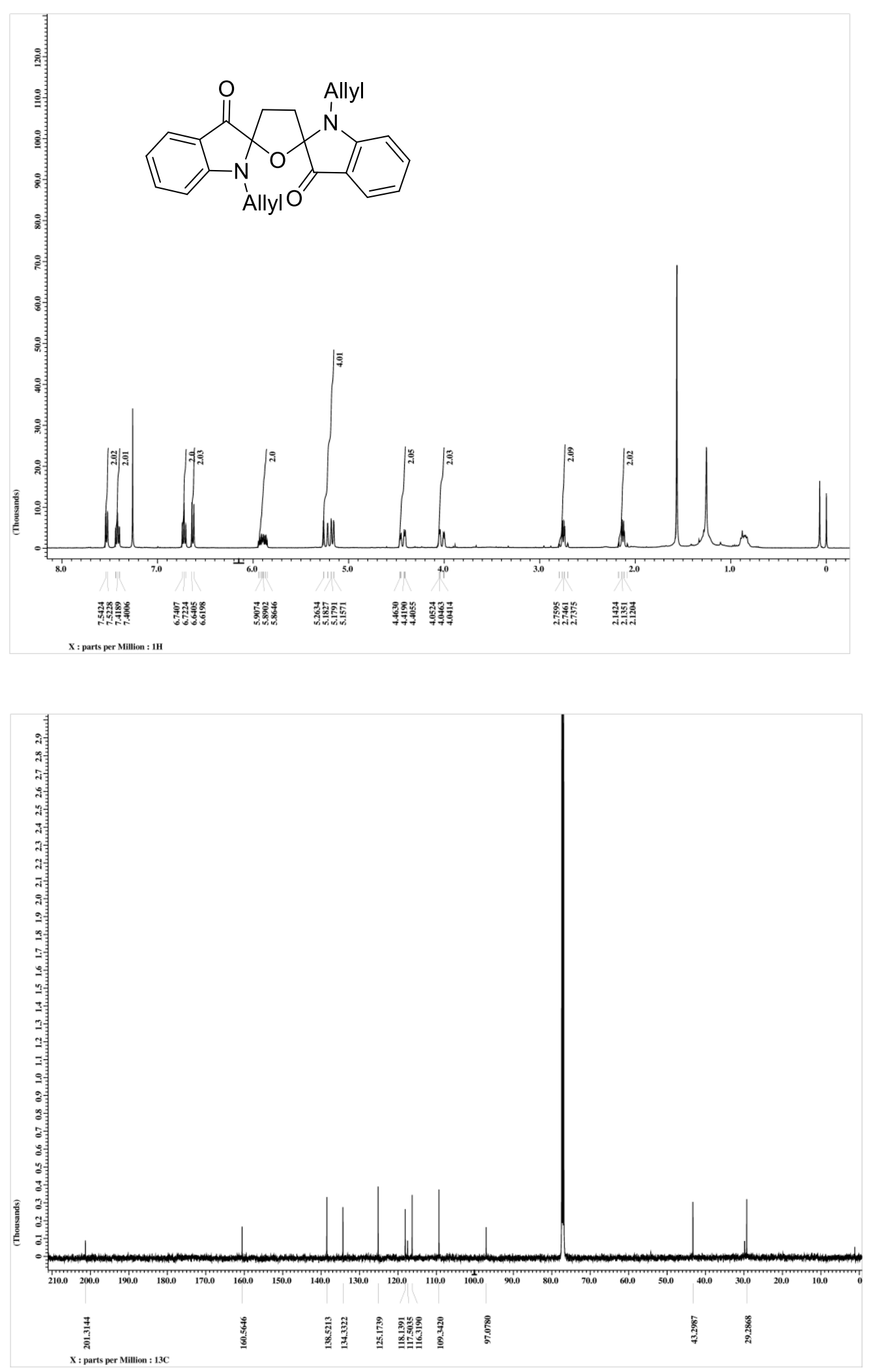